\title{
Dynamic Control of Thermal Transport via Phonon Scattering at Ferroelastic Domain Walls
}

\author{
A Dissertation Presented to the \\ Faculty of the School of Engineering and Applied Science \\ University of Virginia \\ In Partial Fulfillment \\ of the Requirements for the Degree of \\ Doctor of Philosophy \\ Mechanical and Aerospace Engineering
}

Brian M. Foley

September $26^{\text {th }}, 2016$

CCopyright by Brian Foley 2016

All Rights Reserved 
APPROVAL SHEET

The dissertation is submitted in partial fulfillment of the requirements for the degree of

Doctor of Philosophy in Mechanical and Aerospace Engineering

Brian M. Foley

This dissertation has been read and approved by the Examining Committee:

Dean Pamela M. Norris, Chairman

Prof. Patrick E. Hopkins, Advisor

Prof. Jiwei Lu

Prof. Robert M. Weikle

Dr. Jon F. Ihlefeld

Dr. John C. Duda

Accepted for the School of Engineering and Applied Science:

Craig H. Benson, Dean

School of Engineering and Applied Science

September, 2016 


\begin{abstract}
Dynamic control of the thermal properties of a material is the next frontier in nano/macro scale thermal transport. The development of a solid-state "thermal switch" with large dynamic range (ratio of on-state to off-state heat flux) and fast switching times would be a disruptive advancement in the field of on-chip thermal management technologies. Towards this goal, several works have highlighted the concept of dynamic thermal properties in the form of thermal rectification based on geometry alone as well as a combination of geometric and material effects, passing through material phase transitions or modification of the chemical composition. However, none of these approaches have demonstrated the dynamic range, switching speeds or scalability to support the widespread adoption of these technologies into relevant applications.

Building on prior work concerning the scattering of phonons by ferroelastic domain walls in $\mathrm{BiFeO}_{3}$ thin films at room temperature, this work demonstrates active and reversible tuning of the thermal conductivity of lead zirconate titanate $\left(\mathrm{PbZr}_{1-x} \mathrm{Ti}_{x} \mathrm{O}_{3}, \mathrm{PZT}\right)$ by manipulating the domain wall density under applied electric fields. Utilizing a bilayer PZT heterostructure, the thermal conductivity was found to decrease by approximately $11 \%$ under electric fields of $\pm 475 \mathrm{kV} / \mathrm{cm}$. In addition, the change in thermal conductivity with applied field was found to be rapid, reversible and repeatable. If the dynamic range of this thermal switch could be increased well above this $11 \%$ change to $100 \%$ or more, the concept of active thermal management of electronic devices at the nanoscale could become a reality.

In order to optimize the performance of the PZT thermal switch, we must improve our understanding of thermal transport both within the material system and the bilayer heterostructures. This work presents a thorough investigation of the thermal conductivity in both thin film and bulk PZT across the compositional phase diagram, along with an indepth discussion of thermal transport in the component materials $\left(\mathrm{PbTiO}_{3}\right.$ and $\left.\mathrm{PbZrO}_{3}\right)$. The insight gathered from the material study is then applied to bilayer PZT heterostructures where the thermal conductivity is measured as a function of applied electric field using time-domain thermoreflectance. These measurements are conducted on two different bilayer PZT geometries at room temperature and one of the geometries is measured over a temperature range spanning $78-400 \mathrm{~K}$. It is found that the modulation of the domain structure via the applied electric field appears to primarily impact the scattering rates of higher-frequency phonons, with the measured change in thermal conductivity between the zero bias and poled states increasing with temperature. Therefore, if future materials and devices can be engineered to preferentially modulate these high-frequency phonons, then dynamic ranges approaching $100 \%$ or larger may be possible.
\end{abstract}




\section{Acknowledgements}

This dissertation would not have been possible without an enormous amount of help and support from my colleagues, friends and family.

First and foremost, I would like to thank my advisor, Prof. Patrick E. Hopkins. Patrick convinced me to leave a well-paying job and join his new research group at UVA, for which I will be forever grateful. The passion and excitement that Patrick brings to the lab each and every day is truly inspirational and instills a desire in his students to work hard and be their very best. I can confidently say that without Patrick's support, patience and wisdom, I would not be the researcher that I am today. Thank you, Patrick, for being both an amazing advisor, as well as a great friend.

I am very grateful to the members of my dissertation committee for their willingness to help guide my dissertation research; Prof. Pamela Norris, Prof. Robert Weikle, Prof. Jiwei Lu, Dr. Jon Ihlefeld and Dr. John Duda. In particular, I would like to thank Dr. Jon Ihlefeld for all of the samples and guidance that he provided in direct support of my dissertation research. Without the sample fabricated by Dr. Ihlefeld and his team at Sandia National Laboratories, this work would not have been possible.

I would like to express a special thank you to Dr. John Duda for being both a trusted colleague and an amazing friend. Over the past five years, John has been a consistent source of support both technically and personally, discussing phonon transport and the latest indie bands interchangeably. I am looking forward to using my post-dissertation free time to catch up to his skill level on the drums!

A huge thanks to all the members of the ExSiTE lab at UVA both past and present for all the help and support along the way. A special thank you to Dr. Ramez Cheaito for teaching me all about TDTR and being so patient with me during those first few years. We've come a long way since those early years in the old chemistry building when it was just the two of us and I'm so proud to have shared the journey with you, Dr. Cheaito.

An extra special thank you goes to Dr. John Gaskins, Research Scientist in the ExSiTE lab. John was always there to help me in any way that I needed, ranging from help trou- 
bleshooting an experiment to taking a walk around grounds to vent over coffee. I am so thankful that I was able to come to work and spend each day with such an amazing friend and there is no way that I would have made it through my $\mathrm{PhD}$ without you!

Throughout my time in Charlottesville, I have been so fortunate to have had such amazing friends in my life. Many thanks to the Gaskins, the Livermans, the Dooley-Schafers, the Salaways, the Cosners and the Comforts; Chris Petz, Cliff Rowland, Steven Durant, Lauren Mills, Margot Williams, Josh Owings, Zach and Mel Weiner, Dave and Meg Lowe, Chad Williams and Courtney Smart. The memories and moments that I've shared with all of you during my time in Charlottesville will be cherished for the rest of my life.

A special thanks to all of my friends outside of Charlottesville that have lent their support from afar; the Gregoires, the Hardimans, the Foleys, the DeParis, the Donovans, the Brusos, the Mattons, Laura Day, Aleena Bando and the rest of the Worcester Homeloaf. Additionally, a huge thank you to my good friend and fellow WPI alumnus, Hans Jensen.

Last but certainly not least, I would like to thank my amazing family and girlfriend, Caitlin, for all of the love and support that you have provided. To my parents, Jack and Robin: thank you for always being there for me both in good times and in bad, and for molding me into the man that I am today. I love you both so much and am so very proud to be your son. To my sisters, Lindsay and Mara: you are both so important to me and your love and support motivates me to be the best big brother that I possibly can. The three of us make a great team and I love you both so very much. To my extended family, all the Foleys, Mouchons and Collins: thank you all for all your love and support throughout my life.

And to my girlfriend, Caitlin: words can hardly express how much I appreciate you being by my side and supporting me over the past seven years, especially the last five during my $\mathrm{PhD}$. I love you so very, very much and look forward to our next adventure together! 


\section{Contents}

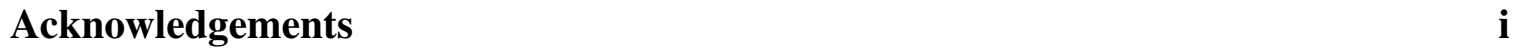

1 Introduction 1

1.1 Passive \& Active Control of Thermal Transport . . . . . . . . . . . . . . . 2

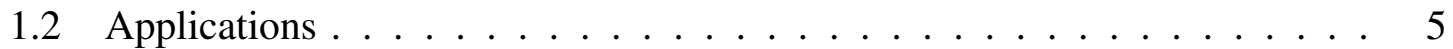

$1.3 \quad$ Statement of Objectives and Scope of Dissertation . . . . . . . . . . . . . . 8

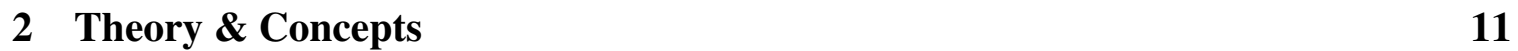

2.1 Phonons . . . . . . . . . . . . . . . . . . . . 11

2.2 Phonon Scattering in Complex Oxides . . . . . . . . . . . . . . . 13

2.2.1 Incoherent Interfaces: Nano-grained $A B \mathrm{O}_{3}$ Perovskites . . . . . . . 13

2.2 .2 Coherent Interfaces: $\mathrm{Sr}_{2} \mathrm{Nb}_{2} \mathrm{O}_{7} \ldots \ldots \ldots \ldots$

2.3 Domains \& Domain Walls/Boundaries . . . . . . . . . . . . . . . . . . 19

2.4 Phonon Scattering at Domain Walls/Boundaries . . . . . . . . . . . . . 22

$\begin{array}{lll}3 & \text { Experimental Methods } & 26\end{array}$

3.1 Time Domain Thermoreflectance (TDTR) . . . . . . . . . . . . . . 26

3.1 .1 System Description . . . . . . . . . . . . . . . . . . 26

3.2 Integration of Electrical Probe Stations . . . . . . . . . . . . . . 28

3.2.1 Room Temperature Probe Station . . . . . . . . . . . . . . . . . 29

3.2 .2 Cryogenic Probe Station . . . . . . . . . . . . . . . 35

3.2 .3 Instrumentation for Electrical Control/Measurement . . . . . . . . 37

4 Thermal Transport in Lead Zirconate Titanate $\left(\mathbf{P b Z r}_{1-x} \mathbf{T i}_{x} \mathbf{O}_{3}\right)$

4.1 Motivation \& Background . . . . . . . . . . . . . 40

4.2 Sample Fabrication \& Characterization. . . . . . . . . . . . . . . . . . . . 43

4.3 Thermal Measurements \& Analysis . . . . . . . . . . . . . . . . . . . . 46

4.4 Results \& Discussion . . . . . . . . . . . . . . . . . . . . . . . . . . . . 48

4.5 Semi-Classical Model for Thermal Conductivity of PZT . . . . . . . . . 53

4.5 .1 Examining trends in bulk $\mathrm{PbTiO}_{3}$ and $\mathrm{PbZrO}_{3} \ldots \ldots$. . . . . . . 54

4.5.2 Thermal conductivity of $\mathrm{PbZr}_{1-x} \mathrm{Ti}_{x} \mathrm{O}_{3}$ as a function of $x$. . . . . . 60

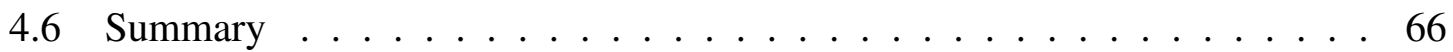


5 Phononic Thermal Switch via PZT Bilayer Structure $\quad 68$

5.1 Bilayer PZT Thermal Switch: Room Temperature . . . . . . . . . . . . . . 69

5.1 .1 Sample Fabrication \& Characterization . . . . . . . . . . . . . . . 69

5.1.2 Thermal Measurements \& Analysis . . . . . . . . . . . . . . . 70

5.1 .3 Results \& Discussion . . . . . . . . . . . . . . . . . . . . 76

5.2 Bilayer PZT Thermal Switch: Temp. Dependence . . . . . . . . . . . . . . 79

5.2.1 Sample Fabrication \& Characterization . . . . . . . . . . . . . 80

5.2 .2 Thermal Measurements \& Analysis . . . . . . . . . . . . . . . . . 81

5.2 .3 Results \& Discussion . . . . . . . . . . . . . . . . . . . . . . . . . . . . . . 82

5.3 Summary . . . . . . . . . . . . . . . . . 88

6 Summary, Impact \& Future Work 90

6.1 Summary . . . . . . . . . . . . . . . . . . . 90

6.2 Impact . . . . . . . . . . . . . . . . . . . . . . . . 92

6.3 Future Work . . . . . . . . . . . . . . . . . . . . . . . . 94

\begin{tabular}{ll}
\hline Bibliography & 95
\end{tabular}

\begin{tabular}{ll}
\hline Appendix A & 105
\end{tabular} 


\section{List of Figures}

1.1 Thermal conductivity as a function of temperature for several $\mathrm{VO}_{2}$ thin films, in-



2.1 (a) Plan and (b) cross sectional SEM micrographs of as crystallized $\mathrm{SrTiO}_{3}$ films on (0001) sapphire. Plan view images of films that were post annealed at (c) 700 , (d) 800 , (e) 900 , and (f) $1000^{\circ} \mathrm{C}$ demonstrate the degree to which post crystallization anneals impact the film microstructures. [4] (g) Thermal conductivity of nano-grained $\mathrm{SrTiO}_{3}\left(\mathrm{ng}-\mathrm{SrTiO}_{3}\right)$ and nano-grained $\mathrm{BaTiO}_{3}\left(\mathrm{ng}-\mathrm{BaTiO}_{3}\right)$ as a function of average grain size at room temperature. Also plotted are models for the thermal conductivity as a function of grain size derived from kinetic theory. [4, 5] 14

2.2 (a) Thermal conductivity versus temperature for two $5 \% \mathrm{La}(\mathrm{x}=0.1) \mathrm{Sr}_{2-x} \mathrm{La}_{x} \mathrm{Nb}_{2} \mathrm{O}_{7-\delta}$ samples of different thicknesses; $130 \mathrm{~nm}$ (blue circles) and $800 \mathrm{~nm}$ (red squares). Literature data for amorphous $\mathrm{SiO}_{2}$ (dashed line, [6]), bulk $\mathrm{Sr}_{5} \mathrm{Nb}_{5} \mathrm{O}_{17}$ (solid line, [7]), $\mathrm{CsBiNb}_{2} \mathrm{O}_{7}$ (triangles, citecahill2010aa) and $\mathrm{WSe}_{2}$ (diamonds, [8]) are included for comparison. (b) Atomic-resolution HAADF-STEM images illustrating the layered crystal structure of strontium niobate. Arrows along the left image indicate the position of defects in the layering sequence. The image on the right shows a higher magnification of a section of the grain overlaid with $\langle 101\rangle$ projections of the $\mathrm{Sr}$ (yellow) and $\mathrm{Nb}$ (orange) atom positions in the $\mathrm{Sr}_{2} \mathrm{Nb}_{2} \mathrm{O}_{7}(n=4$ $\mathrm{NbO}_{6}$ octahedra $)$ and $\mathrm{Sr}_{5} \mathrm{Nb}_{5} \mathrm{O}_{17}\left(n=5 \mathrm{NbO}_{6}\right.$ octahedra $)$ crystal structures. [9] . . 17 
2.3 Thermal conductivity versus temperature for two $5 \% \mathrm{La}(x=0.1) \mathrm{Sr}_{2-x} \mathrm{La}_{x} \mathrm{Nb}_{2} \mathrm{O}_{7-\delta}$ samples of different thicknesses plotted along with two models for the minimum thermal conductivity of a solid; the Cahill-Watson-Pohl (CWP) model [10] and a modified version of the CWP that includes the effects of scattering between weakly-bonded layers (dashed line, this work). . . . . . . . . . . . . . . 18

2.4 (a) Diagram of the tetragonal PZT unit cell, illustrating the distortion along the $c$ axis at temperatures below the Curie temperature $\left(T_{C}\right)$ that results in a spontaneous polarization vector, $\overrightarrow{\mathrm{P}}$. (b) Atomic-resolution, aberration-corrected transmission electron microscopy (TEM) image illustrating the displacement of the central Bsite atom, and thus the local spontaneous polarization vectors in a $\mathrm{PbZr}_{0.2} \mathrm{Ti}_{0.8} \mathrm{O}_{3}$ thin film [11]. The large yellow arrows denote the average polarization direction in each region (domain) of the crystal separated by the dashed lines. These dashed lines correspond to different types of domain walls separating these domain regions, including ferroelectric walls (yellow dashes) and ferroelastic walls (blue dashes). (c) Plan-view channeling-contrast scanning electron microscopy (CCSEM) image of a bilayer PZT thin film studied in this dissertation, depicting the complex arrangement of ultra-fine nano-domains bounded by ferroelastic domain

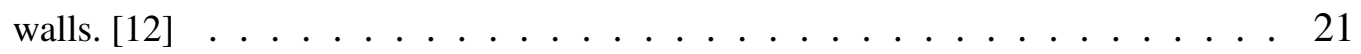

2.5 Thermal conductivity of $\mathrm{BaTiO}_{3}$ single crystals from 3 - $50 \mathrm{~K}$, measured in [13]. Open circles denote measurements made on the unpoled crystals, while open triangles denote measurements made while the samples were actively poled by an 11 $\mathrm{kV} \mathrm{cm}^{-1}$ electric field. . . . . . . . . . . . . . 23

2.6 (a) Effective thermal conductivities versus temperature for $\mathrm{BiFeO}_{3}$ films with different numbers of domain variants, and thus, different domain wall densities, along with literature data for bulk $\mathrm{SrTiO}_{3}$ (open squares, Refs. [14, 15]) and amorphous $\mathrm{SiO}_{2}$ (solid line, Ref. [6]) for context. (b,c) Plan-view piezoresponse force microscopy (PFM) images illustrating the increase in domain wall density in the 4-domain variant film compared to the 2-domain variant film. The decrease in the thermal conductivity observed in the 4-domain variant case in (a) is attributed to this increase in domain wall density. [16] . . . . . . . . . . . . . . . . . 24

3.1 Left: Block diagram of the two-color time-domain thermoreflectance (TDTR) system at the University of Virginia. Right: Illustration of the TDTR ratio data being fit to the multilayer thermal model to extract the thermal transport parameters of interest for a given sample. [17] . . . . . . . . . . . . . . . . . . 27

3.2 Photograph of the LMS-2709 Microprobing Station manufactured by J microTechnology, Inc., prior to the modifications that were made to integrate the unit into the TDTR system. . . . . . . . . . . . . . . 30 
3.3 (a) Photograph of the modified LMS-2709 probe station integrated into the TDTR system. The path of the coaxial pump and probe beam to the sample sample is highlighted to illustrate the addition optics required to periscope the beam up, as well as direct the beam downward towards the sample mounted on the chuck. Also pictured is the imaging system comprised on a stereo microscope and camera, mounted at an angle to enable accurate placement of the electrical probes onto the top electrodes/thin films. Close-up images of the modified probe station (b) showing the optics included to route the laser beam at normal incidence to the sample surface, as well as the kinematic mount (c) added to the chuck to adjust the planarity of the sample and reflect the beam back to the photo-detector. Images of the sample surface showing an electrical probe tip in contact with a top electrode on a PZT sample, both without (d) and with (e) the laser focused on the electrode. . 31

3.4 (a) Photo of the room temperature probe station making a measurement on one of the functionalized SLG samples from the study. (b) Plan-view diagram of the TLM contact geometry for the in-plane electrical measurements, showing the functional groups underneath the gold pads. (c) Optical plan-view image of the TLM geometry used in this study after fabrication, including the measured distances between pads. [18] . . . . . . . . . . . . . . . . . . . . . . . . . 33

3.5 Width-normalized contact resistivity $\left(\rho_{\mathrm{C}}\right)$ versus atomic percent (at \%) coverage of various adsorbates on single layer graphene. [18] The introduction of oxygen or nitrogen at coverages greater than 5 at $\%$ causes $\rho_{\mathrm{C}}$ to increase dramatically. Conversely, while low coverages of $\approx 5$ at $\%$ fluorine causes an increase in $\rho_{\mathrm{C}}$, coverages greater than 5 at $\%$ cause $\rho_{\mathrm{C}}$ to decrease with increasing coverage. The dashed lines represent contact resistivity values reported for Au/Ti/SLG [19] and palladium/SLG [20], respectively. Inset: Sheet resistance $\left(R_{\mathrm{S}}\right)$ of the graphene between the TLM pads due to doping of the graphene adjacent to the metal contacts that occurred while functionalizing the graphene via physical masks. . . . . . . . 34

3.6 (a) Photograph of the ST-500 Cryogenic Probe System manufactured by Janis Research Company, LLC. Inset: View of the sample chuck and probe tips within the ST-500 through the top-side optical window. (b) Photograph of ST-500 integrated into the TDTR system, complete with all peripheral equipment required to perform temperature control and electrical bias for the measurements. . . . . . . 35

3.7 (a) Photograph of the ST-500 within the TDTR system, highlighting the optical components required to route the beam over to and down into the probe station. (b) Additional photograph showing the imaging system used to ensure accurate placement of the probe tips on the samples mounted in the cryogenic probe station, as well as the triplet lens required due to the large internal distance between the sample surface and the top optical window. . . . . . . . . . . . . 36

3.8 Example of Polarization vs. Electric Field (P-E) loop data collected from a PZT device to illustrate important features of the hysteresis curve, including the coercive field $\left( \pm \mathrm{E}_{\mathrm{c}}\right)$, saturation polarization $\left( \pm \mathrm{P}_{\mathrm{sat}}\right)$ and remanent polarization $\left( \pm \mathrm{P}_{\mathrm{r}}\right)$. Arrows indicate the progression of the applied electric field, sweeping from zero field to maximum positive field, then maximum negative field, and then back to zero field. . . . . . . . . . . . . . . . 39 
$4.1 \quad$ Phase diagram of lead zirconate titanate $\left(\mathrm{PbZr}_{1-x} \mathrm{Ti}_{x} \mathrm{O}_{3}, \mathrm{PZT}\right)$, adapted from [21]. . 41

4.2 Representative $2 \theta-\theta$ XRD analysis of PZT thin films with compositions (i) $\mathrm{PbZr}_{0.30} \mathrm{Ti}_{0.70} \mathrm{O}_{3}, \mathrm{PbZr}_{0.52} \mathrm{Ti}_{0.48} \mathrm{O}_{3}$ at pyrolysis temperatures of (ii) $350^{\circ} \mathrm{C}$ and (iii) $400^{\circ} \mathrm{C}$ and (iv) $\mathrm{PbZr}_{0.70} \mathrm{Ti}_{0.30} \mathrm{O}_{3}$. Compositions near the MPB with a pyrolysis temperature of $350^{\circ} \mathrm{C}$ were characterized as randomly oriented, while compositions with a pyrolysis temperature of $400^{\circ} \mathrm{C}$ possessed a (100)-texture. . . . . . . 43

4.3 Plan-view (a,b) and cross-sectional (c,d) scanning electron microscopy (SEM) images of the MPB-composition thin films at the two pyrolysis temperatures, highlighting the significant difference in average grain size. . . . . . . . . . . . . 44

4.4 Thermal conductivity $(\kappa)$ of PZT thin films with various solid-solution compositions, $x$, fabricated with pyrolysis temperatures of $350^{\circ} \mathrm{C}$ (blue squares) and $400^{\circ} \mathrm{C}$ (red circles). The dashed vertical lines denote the locations of the phase boundaries from Fig. 4.1 at $20^{\circ} \mathrm{C}$. . . . . . . . . . . . . . . . . . . . . . 48

4.5 Bulk thermal conductivity data over temperature for $\mathrm{PbTiO}_{3}$ ([22], blue line) and $\mathrm{PbZrO}_{3}([23,24]$, red circles) highlighting the large difference in the temperature trends between stoichiometrically-similar materials $\left(\mathrm{ABO}_{3}\right.$ perovskite oxides). Also plotted for comparison are $\mathrm{SrTiO}_{3}$ ([15], open squares), $\mathrm{NaNbO}_{3}$ ([22], open circles) and $a-\mathrm{SiO}_{2}([6]$, black line). . . . . . . . . . . . . 50

4.6 Thermal conductivity of bulk PZT of various compositions. Samples measured in this work (black diamonds) include $\mathrm{PbZrO}_{3}(x=0), 70 / 30$ PZT $(x=0.30)$ and $30 / 70$ PZT $(x=0.70)$. Values measured in the literature include $\mathrm{PbTiO}_{3}[22](x=$ 1 , white diamond) and bulk data for samples around ([25], upward triangles) and at $([26]$, downward triangle) the MPB. . . . . . . . . . . . . . . . 51

\begin{tabular}{|lll|l|l|}
\hline 4.7 & Bulk thermal conductivity data for $\mathrm{PbTiO}_{3}$ from [22] used to fit Eq. & 4.2 & in deter-
\end{tabular} mining the value of the intrinsic scattering constants $\left(A_{\mathrm{LA}, \mathrm{PTO}}, A_{\mathrm{TA}, \mathrm{PTO}}, B_{\mathrm{LA}, \mathrm{PTO}}\right.$, $\left.B_{\mathrm{TA}, \mathrm{PTO}}, C_{\mathrm{LA}, \mathrm{PTO}}, C_{\mathrm{TA}, \mathrm{PTO}}\right)$ for the material. $\ldots \ldots \ldots \ldots 6$

4.8 Bulk thermal conductivity data for $\mathrm{PbTiO}_{3}$ from [22] and $\mathrm{PbZrO}_{3}$ from [23, 24], \begin{tabular}{|l|l|l|}
\hline along with calculated models for each material using Eq. & 4.2. & Also plotted is the
\end{tabular}

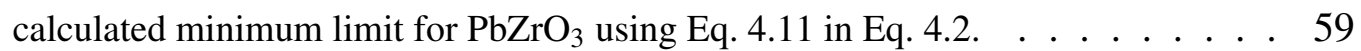

4.9 Bulk thermal conductivity data for $\mathrm{PbZrO}_{3}$ from [23, 24] and measured values for 30/70 PZT thin films ( $x=0.7,325 \mathrm{~nm}$ thick) over temperature. Also plotted is the calculated minimum limit for $\mathrm{PbZrO}_{3}$ using Eq. 4.11 in Eq. $4.12 \mid$ with $x=0$. \begin{tabular}{ll|l|l|l|}
\hline The models represent fits to the data defined by Eqs. 4.12 & through & 4.17 & treating
\end{tabular} $A$ and $d$ as adjustable parameters to gain insight regarding the phonon scattering processes in these samples. . . . . . . . . . . . . . 63

\begin{tabular}{|ll|l|l|l|}
\hline 4.10 & Thermal conductivity of PZT thin films plotted with the model from Eq. & The
\end{tabular} data and model related to samples pyrolyzed at $350^{\circ} \mathrm{C}$ are denoted by blue squares and dashed blue line, respectively, while the data for samples pyrolyzed at $400^{\circ} \mathrm{C}$ are denoted by red circles. . . . . . . . . . . . . . . . . 65

$5.1 \quad$ (a) Experimental geometry showing cross section of PZT bilayer sample. (b) Cross-section bright-field transmission electron micrograph (TEM) of a PZT bilayer film showing a stripe pattern of $90^{\circ}$ ferroelastic domains in the upper $\mathrm{PbZr}_{0.3} \mathrm{Ti}_{0.7} \mathrm{O}_{3}$

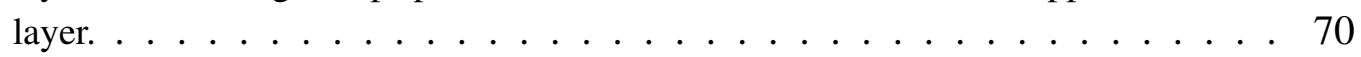


5.2 Sensitivity of the four-layer thermal model to various parameters at a modulation frequency of $2.57 \mathrm{MHz} . h_{\mathrm{k}, 1}$ is the Kapitza conductance between layers 1 and $2 ; C_{\mathrm{v} 2}$ is the heat capacity of layer $2\left(30 / 70\right.$ PZT); $\kappa_{2}$ and $\kappa_{3}$ are the thermal conductivities of layer 2 and layer 3 (70/30 PZT), respectively; $h_{\mathrm{k}, 3}$ is the Kapitza conductance between layer 3 and the remaining layers beneath. . . . . . . . . . . 72

5.3 (a) Thermal conductivity versus applied electric field for a PZT bilayer device. The thermal conductivity decreases with increasing electric field magnitude due to the increase in domain wall density that impedes phonon transport within the film. [12] (b) Real-time measurement of the thermal conductivity of the PZT bilayer at a fixed TDTR delay time of $150 \mathrm{ps}$ as the applied bias is cycled five times between the no-field and high-field $(10 \mathrm{~V}, 475 \mathrm{kV} / \mathrm{cm})$ conditions. [12] . . . . . . . . . . . 77

5.4 Real-time thermoreflectance signal measured via TDTR at a fixed delay of 150 ps for a platinum $/ a-\mathrm{Al}_{2} \mathrm{O}_{3} /$ platinum metal-insulator-metal structure as the applied electric field is cycled between $0 \mathrm{~V}(0 \mathrm{kV} / \mathrm{cm})$ and $\pm 5 \mathrm{~V}( \pm 370 \mathrm{kV} / \mathrm{cm})$. No changes are observed in the thermoreflectance signal, indicating that the response exhibited by the PZT bilayer films is unique to that structure. . . . . . . . . . . 79

5.5 (a) Diagram of the 1x1 bilayer PZT heterostructure measured as a function of temperature at zero and $-450 \mathrm{kV} \mathrm{cm}^{-1}$ applied electric fields. (b) Cross-sectional SEM image of the 1x1 bilayer heterostructure. Note the striped-contrast of the ferroelastic domains in the top tetragonal $\mathrm{PbZr}_{0.3} \mathrm{Ti}_{0.7} \mathrm{O}_{3}$ layer that are not present in the bottom rhombohedral $\mathrm{PbZr}_{0.7} \mathrm{Ti}_{0.3} \mathrm{O}_{3}$ layer. (c) Plan-view SEM image os the 1x1 bilayer heterostructure, illustrating the anomalously large grain sizes and complex, ultra-fine ferroelastic domain structure of these bilayers. [12, 27, 28] . . 80

5.6 Thermal conductivity versus applied electric field measured at room temperature for the 1x1 bilayer PZT device. As observed in the 2x1 PZT heterostructure from [12], the thermal conductivity decreases with increasing electric field magnitude, likely due to the increase in domain wall density that increases the phonon scattering rate within the film. . . . . . . . . . . . . . . 81

5.7 Thermal conductivity of the top tetragonal 30/70 PZT layer in the 1x1 bilayer PZT heterostructure over a temperature range of 78 - $400 \mathrm{~K}$. Measurements were made at the same spot on the sample at every temperature, both with $\left(-450 \mathrm{kV} \mathrm{cm}^{-1}\right.$, red squares) and without $\left(0 \mathrm{kV} \mathrm{cm}^{-1}\right.$, black squares $)$ an applied electric field. As the temperature increases, the percent change in the thermal conductivity when under bias also increases. . . . . . . . . . . . . . . . . 83

5.8 Polarization versus electric field (P-E) hysteresis loops measured for the 1x1 bilayer PZT heterostructure at several different temperatures from $78-450 \mathrm{~K}$. The data confirms that the $-450 \mathrm{kV} \mathrm{cm}^{-1}$ applied electric fields were indeed large enough to switch the domain structure at all the temperatures tested with TDTR. Furthermore, the value for the coercive field in the negative poling direction $\left(-\mathrm{E}_{\mathrm{c}}\right)$ decreases with increasing temperature, indicating that lower fields are required to switch the domain structure at elevated temperatures. . . . . . . . . . . . . . . . 84 
5.9 Measured thermal conductivity versus temperature for the single layer 30/70 PZT thin film from Chapter 4 along with the data for the top tetragonal 30/70 PZT layer in the bilayer heterostructure. Also plotted is the calculated minimum limit for 30/70 PZT using Eq. 4.11|in Eq.|4.12|with $x=0.7$. The models represent fits to the data defined by Eqs. 4.12 through 4.17 , treating $A$ and $d$ as adjustable parameters to gain insight regarding the phonon scattering processes in these samples. . . . . 85

5.10 Thermal conductivity versus temperature of a 1x1 bilayer PZT device both with and without an applied electric field, along with fits to the data defined by Eqs. 4.12 through 4 .17, treating $A$ and $d$ as adjustable parameters to gain insight regarding the phonon scattering processes in these samples. The change in thermal conductivity due to the applied field increases as temperature increases, suggesting that the modulation of the fine ferroelastic domain structure has a significant impact on the short-wavelength phonons moving through the bilayer. . . . . . . . . . . 87

$1 \quad$ Diagram of the $3 \times 1$ bilayer PZT device used in these TDTR measurements with multiple thermal modulation frequencies. . . . . . . . . . . . . . 105

$2 \quad$ (a) Diagram of the relationship between thermal penetration depth, $\delta$, and thermal modulation frequency; as the thermal modulation frequency increases, $1 / e$ value of $\delta$ decreases. (b) 2D surface of TDTR ratio data collected by making full TDTR scans at fifteen modulation frequencies ranging from $700 \mathrm{kHz}$ to $9 \mathrm{MHz}$. . . . . . 106

3 Percent change in the 2D TDTR ratio surface compared to data collected on an unpoled bilayer PZT device both (a) during active bias and (b) after the bias is removed. During active poling of the bilayer PZT device, the largest changes in the TDTR ratio surface appear to be at the shallow and deeper penetration depths (highest and lowest modulation frequencies, respectively), with maximum changes of $-3 \%$ observed. Little to no change appears to occur in the central modulation depths of the range. When the electric field is removed, the TDTR signals at deeper penetration depths (lower frequencies) recover to their unpoled levels (i.e., the percent change goes to zero) while the TDTR signals at the shallower penetration depths do not recover to their unpoled levels. This suggests that the features of the bilayer PZT device that cause the changes at deeper depths are reversible in nature while the changes at shallower depths are irreversible. . . . . . . . . . . . 107

$4 \quad$ Sensitivity of the 4-layer thermal model detailed in Chapter 4 to $10 \%$ decreases in both (a) the thermal conductivity of the top tetragonal PZT layer $\left(\kappa_{2}\right)$ and (b) the bottom rhombohedral PZT layer $\left(\kappa_{3}\right)$. While these results indicate that the large changes in TDTR signal at deeper penetration depths can be due to changes in either layer, the changes at shallower depths are likely to be due to changes in the top tetragonal PZT layer and not the bottom layer, meaning that the irreversible change in the 2D TDTR ratio surface in Figure 3 (b) is likely due to the ferroelastic domain structure in the top tetragonal layer only. . . . . . . . . . . . . . . . 109 


\section{List of Tables}

4.1 Pyrolysis temperature $\left(T_{\mathrm{P}}\right)$, film thickness $\left(d_{f}\right)$ and average grain sizes $\left(d_{g}\right)$, along with the associated measurement uncertainties $\left(\sigma_{d}, \sigma_{g}\right)$.

4.2 Material properties for $\mathrm{PbZrO}_{3}$ and $\mathrm{PbTiO}_{3}$ used in the model for $\kappa(x)$

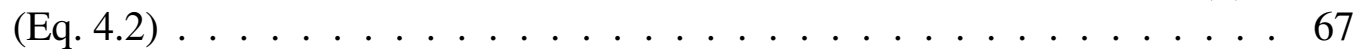

5.1 Fixed values employed or the 4-layer model when fitting the TDTR data acquired from the PZT bilayer samples while under applied electric fields. 73 


\section{Chapter 1}

\section{Introduction}

Dynamic control of the thermal properties is the next frontier in nano/macro scale thermal transport. Up to now, the primary objectives for controlling the flow of heat have been focused on making a material or interface as conductive or resistive as possible. Towards these goals, a tremendous amount of research has been conducted to engineer new materials and optimize the thermal transport properties of a particular device or system. Furthermore, these advancements have been engineered with the aim that the heat-carrying abilities change as little as possible over their operational lifetime, particularly when subjected to various extreme environmental conditions (temperature, pressure, etc). These design criteria for the control of thermal energy have helped revolutionize technologies in a wide variety of fields (e.g., stable, insulative coatings for aerospace/exoatmospheric components; cooling systems for electronics, etc.).

As we enter an age where energy availability and efficient usage are of paramount concern to an increasingly global society, the need for dynamically-tunable, "smart" materials is greater than ever. The ability to tune a particular device or subcomponent to maximize its performance based on the operating conditions is a core component of the progress we have made to date towards making systems as efficient as possible. Towards these goals, the past few decades have been rife with great advances in a variety of fields (electronic, structural and chemical, to name a few) where components can dynamically respond to 
external stimuli and provide immediate feedback to, or control of an operational system. By comparison, the field of thermal management remains rather antiquated with the idea of dynamic, tunable thermophysical properties gaining attention only relatively recently.

To date, an immense amount of work has focused on the impact of microstructure on the thermal conductivity of a material as the characteristic length scales of the structure become comparable to the mean free paths of the thermal energy carriers [29, 30]. The thermal conductivity, $\kappa\left(\mathrm{W} \mathrm{m}^{-1} \mathrm{~K}^{-1}\right)$, of a solid is a temperature dependent material property that relates the temperature difference across a section of a material to the heat flux flowing through it and can be empirically described by Fourier's law:

$$
q=-\kappa \nabla T
$$

where $q$ is the heat flux $\left(\mathrm{W} \mathrm{m}^{-2}\right)$ and $\nabla T$ is the temperature gradient $(\mathrm{K})$. The ability to tailor $\kappa$ of a material due to film thickness, grain size and impurity/vacancy concentration has played a major role in the spike of research that has occurred over the past two decades. In most cases, these structural attributes that affect the phonon mean free paths within a given material cannot be easily changed unless they are exposed to environmental extremes (temperature, pressure, etc). However, if the scattering length scales present in the material could be changed, it may be possible to modulate the phonon flux within the material on the basis of scattering separate phonon populations in different mean free path regimes. If realized, the development of a solid-state "thermal switch" with large dynamic range and fast switching times would be a disruptive advancement with the potential to be comparable in scope to the impact of the solid-state transistor.

\subsection{Passive \& Active Control of Thermal Transport}

The research conducted to date in this area can be broadly categorized according to the ability to either passively or actively control thermal transport. In this particular context of heat flow, a passive system is defined as one in which only thermal fields dictate the 
transport properties of the material and/or device, whereas active systems involve external control through a non-thermal stimuli (e.g., electric field, magnetic field, etc). A prime example of a passive system is the idea of thermal rectification through means of a "thermal diode". Thermal rectification via geometric asymmetry in carbon and boron nitride nanotubes that were inhomogenously mass-loaded with heavy molecules [31] has been demonstrated with factors of up to $7 \%$ via energy transport by solitons (self-reinforcing solitary waves that maintain their shape while propagating through a material with a constant velocity). While the applicability to power/energy harvesting systems is limited due to the small degree of rectification, the authors highlighted the use of thermal rectification to start considering phonons as information carriers similar to electrons and photons.

More recently, thermal rectification via photons was demonstrated [32] through the use of a "trapping" methodology comprised of pyramidal reflectors and an inelastic thermal collimator, yielding thermal rectification of around $11 \%$. Such a device could serve as a critical component in solar-thermal power systems providing a variety of functions such as boosting efficiency of individual power generators, as well as improved management of thermal power flow within a grid-scale power inversion unit connected to an array of power generators.

Material phase transitions have been employed as a method to change $\kappa$, often in response to a change in temperature. One example of this is the change in $\kappa$ of vanadium dioxide $\left(\mathrm{VO}_{2}\right)$ thin films due to a metal-insulator transition (MIT) which occurs at a specific temperature [1]. Figure 1.1 plots the results from Ref. [1] along with separate $\mathrm{VO}_{2}$ samples fabricated and measured at the University of Virginia [2]. Upon elevation of the material temperature above a critical point (often 330-350 K depending on phase purity, doping, etc.), the number of free carriers within the material increases by 2-3 orders of magnitude causing a comparable increase in the electrical conductivity. This increase in the materials electrical conductivity also results in an increase of the electronic-contribution to $\kappa$ via the Wiedemann-Franz law, with total increases of up to $60 \%$ having been demonstrated experimentally [1]. 


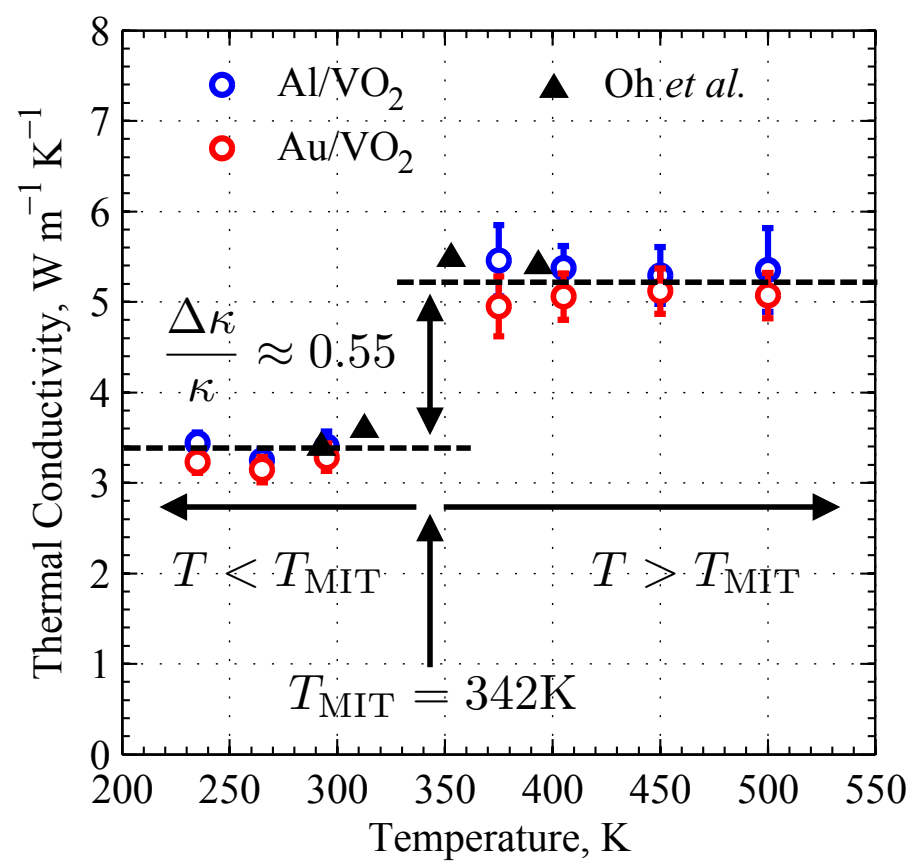

Figure 1.1: Thermal conductivity as a function of temperature for several $\mathrm{VO}_{2}$ thin films, including the published results from Oh et al. in Ref. [1], as well as unpublished measurements made by the author [2]. As the sample temperature is elevated across the metal-insulator transition (MIT) temperature, the thermal conductivity increases by $55-60 \%$.

Another example employing phase transitions is the use of a solid-liquid transition in a hexadecane/graphite suspension [33]. In this work, it was shown that the electrical and thermal conductivities exhibit large increases (approx. 100x and 3.2x, respectively) as the suspension is cooled and transitions from a liquid (low-conductivity) state to a solid (highconductivity) state. The large changes in these properties were attributed to the reduction in the contact resistance between the graphite flakes as the solution solidified, thereby enhancing electronic conduction through the matrix. The changes in electrical and thermal conductivity were also shown to be reversible and repeatable upon cycling the temperatures above and below the critical temperature of $18^{\circ} \mathrm{C}$.

Researchers have also worked to combine the aforementioned approaches in an effort to develop expanded functionality in thermal devices. Temperature-gated rectification has been demonstrated [34] by applying similar geometric considerations to those in Ref. [31] in combination with the MIT in $\mathrm{VO}_{2}$. This work presented a "three-terminal" device in 
which a tapered, suspended beam of $\mathrm{VO}_{2}$ could be switched between rectifying and resistive heat conduction modes by varying the temperature of the device around the critical MIT temperature $\left(T_{\mathrm{MIT}}\right)$. At temperatures below $T_{\mathrm{MIT}}$, the devices studied exhibit thermal rectification by anywhere from 6-28\% while at temperatures above $T_{\mathrm{MIT}}$, the rectifying behavior is significantly suppressed. While these results are indeed exciting from a fundamental perspective, the scalability of $\mathrm{VO}_{2}$ nanobeams, as well as the problem of thermal-runaway are serious impediments towards the perceived energy harvesting applications.

One of the few thermal devices in which control of the transport properties was performed by a non-thermal stimuli was the demonstration of tunable thermal conductivity in $\mathrm{Li}_{x} \mathrm{CoO}_{2}$ thin films via electrochemical lithiation [35]. By charging and discharging the structure via galvanostatic cycling to control the degree of lithiation $(x=1.0-0.6)$, the thermal conductivity of the material was changed from 5.4 to $3.7 \mathrm{~W} \mathrm{~m}^{-1} \mathrm{~K}^{-1}$ which the authors relate to commensurate change in the elastic modulus of the material. As with the hexadecane/graphite solutions in Ref. [33], the control of the thermal conductivity was shown to be reversible and repeatable, in this case on the order of 4-5 hours per period.

While these prior works have laid the foundation of fundamental research into thermal devices, none of these approaches have demonstrated the combination of dynamic range (ratio of on-state to off-state heat flux), switching speed and scalability to support the widespread adoption of these technologies into relevant applications. In order for thermal devices to make a real impact outside the laboratory, these design criteria must be considered en ensemble as researchers develop new materials and/or device topologies.

\subsection{Applications}

\section{On-Chip Active Thermal Management}

One of the most interesting applications of solid-state thermal switches is the idea of on-chip and/or on-die thermal management. In certain integrated circuit topologies such as those associated with high-end sensor, detector or transducer technologies, maintain- 
ing a stable temperature can be critical to minimize errors associated with the temperature dependent properties of the sensing element. In many cases, these high-end devices are fabricated on the micro-to-nanoscale to maximize certain performance metrics (sensitivity, response time, etc), which can often mean that the actual device is built upon layers of ancillary material layers that are either necessary for fabrication (i.e., lattice matching for epitaxial film growth) or for operation (metallic interconnects, semi-insulating layers, etc). Each of these layers adds additional thermal resistance and thermal mass between the functional device and the thermal ground of the die (often the face of the die attached to the package), thereby affecting both the maximum heat flux that can be drawn from a chip while keeping the die at an acceptable temperature as well as the thermal time constant for closed-loop temperature control systems. In short, the benefits of larger heat-sinks and forced-fluid cooling are irrelevant when it comes to providing active temperature regulation of individual devices at the nanoscale.

An alternative would be to fabricate a switchable thermal layer within the structure of the die, ideally as close to the sensing element as possible. Under the application of an external stimuli that is independent of the thermal system (i.e., electric or magnetic field), the thermal conductivity would switch between one of two possible states; a highconductivity, high heat flux 'ON' state and a low-conductivity, low heat flux 'OFF' state. If the heat flux flowing from the sensing element to thermal ground can be either amplitude and/or frequency modulated using the switchable thermal layer, then the temperature of the sensing element could be actively controlled/stabilized as part of a closed-loop system in response to fluctuations in either the power delivered to the sensing element or changes in the ambient operating temperature. This temperature regulation topology is analogous to operation of switching voltage regulators which use pulse width modulation (PWM) control schemes to regulate their output voltage at a fixed value irrespective of fluctuations in either the input voltage to the regulator or the load resistance drawing power from it. 


\section{Improved Thermoelectric Efficiency via Modulated Heating}

Another application has to do with the concept of improving the efficiency of existing thermoelectrical energy converters. In a paper by Yan and Malen (Ref. [3]), it was demonstrated that the conversion efficiency can by increased by $140-180 \%$ by periodically heating the device with either a sinusoidal or square waveform, respectively, and further improvements were possible through the use of low duty-cycle pulsed periodic sources. The experiment was conducted by mounting a resistive Joule heater to a commercially available thermoelectric power generator and generating the various thermal profiles with a waveform generator. In all, the paper described the possibility of making thermoelectric devices made of materials with thermoelectric figures of merit $(Z T)$ equal to unity perform as if they were made of materials with $Z T=1.6-2.8$.

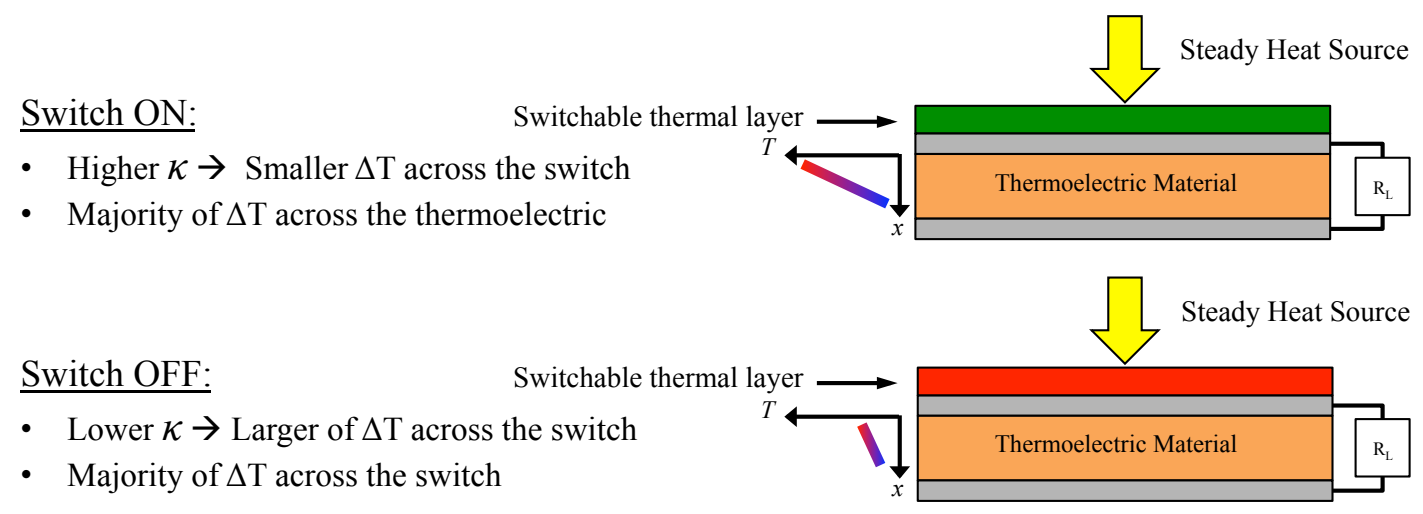

Figure 1.2: Concept of using a switchable thermal layer to modulate the temperature gradient across a thermoelectric generator. In the 'ON' or high- $\kappa$ state, the thermal layer acts ideally as a 'short-circuit' for heat flow and the majority of the thermal gradient $(\nabla T)$ will be across the thermoelectric generator. Conversely, in the 'OFF' or low- $\kappa$ state, the thermal layer acts ideally as an 'open-circuit' with the majority of $\nabla T$ across the switchable layer itself. By cycling the thermal layer between its high and low- $\kappa$ states, the heat flux from the steady source to the thermoelectric generator can be modulated in the manner required to yield the improvements outlined in Ref. [3].

While this is an exciting result, the reality is that there are few regularly periodic heat sources in nature. The paper alludes to this fact and postulates that in order to achieve these improvements in efficiency in real waste heat recovery systems, it will require the development of a viable thermal switch of some sort that will be able to modulate the heat 
flux between a thermal reservoir that can store the energy and the thermoelectric power generator. Figure 1.2 illustrates this concept: placed between the steady heat source and the hot-side of a thermoelectric generator, the switchable thermal layer can act as a valve for the heat flux flowing from source to sink. In the 'ON' or high- $\kappa$ state, the thermal layer acts ideally as a 'short-circuit' for heat flow and the majority of the thermal gradient $(\nabla T)$ will be across the thermoelectric generator. Conversely, in the 'OFF' or low- $\kappa$ state, the thermal layer acts ideally as an 'open-circuit' with the majority of $\nabla T$ across the switchable layer itself. By cycling the thermal layer between its high and low- $\kappa$ states, the heat flux from the steady source to the thermoelectric generator can be modulated in the manner required to yield the improvements outlined in [3]. Such an advancement would provide an enormous boost to the viability of thermoelectric power generators as a large-scale source of distributed, as well as grid-scale sources of electricity.

\subsection{Statement of Objectives and Scope of Dissertation}

This work is focused on understanding the intricacies of phonon transport within both the lead zirconate titanate (PZT) material system as well as bilayer PZT heterostructures. PZT is an incredibly versatile and complex material in its own right and in order to draw conclusions regarding the nature of phonon scattering due to ferroelastic domain walls, it is only prudent that we first understand the intrinsic transport properties of the parent material before addressing extrinsic effects. While this work is focused on a particular material system, the understanding developed from this research is applicable to thermal transport in any material with ferroelastic domain walls $\left(\mathrm{BiFeO}_{3}, \mathrm{BaTiO}_{3}\right.$, etc.). The results of this dissertation will help determine the realistic potential of PZT as a material for thermal devices that can be used in real applications. The dissertation is organized as follows:

- Chapter 2: The core elements of this dissertation are introduced and defined. The concept of a quantized lattice vibration, or phonon is introduced and discussed with a particular focus on fundamental phonon transport in oxide materials. Domains and 
domain boundaries of various types (ferroelectric, ferroelastic, etc.) are defined and their importance regarding the remarkable properties of several materials is examined. Finally, the influence of domains and domain boundaries on phonon transport that has been reported in the literature to date is presented and discussed.

- Chapter 3: The thermal and electrical measurements conducted as part of this dissertation are discussed. The thermal measurements were made using Time Domain Thermoreflectance (TDTR), while the electrical measurements were made using an LCR meter from Keysight Technologies and a ferroelectric tester from Radiant Technologies. An overview of each measurement and the equipment employed is presented. One of the core outcomes of this work was the demonstrated ability to make thermal transport measurements while the materials and/or devices were subjected to an applied electric field. To accomplish this, two types of electrical probing stations (room-temp and cryo-temp) were integrated within the optical measurement system to provide accurate measurements in operando.

- Chapter 4: A thorough investigation of the thermal conductivity $(\kappa)$ in both thin film and bulk lead zirconate titanate $\left(\mathrm{PbZr}_{1-x} \mathrm{Ti}_{x} \mathrm{O}_{3}, \mathrm{PZT}\right)$ across the compositional phase diagram is presented. Since the bilayer-PZT heterostructure is comprised of individual PZT layers with different compositions, it is instructive that we first explore and understand the phonon transport processes within the material itself. The temperature dependent thermal conductivity of bulk $\mathrm{PbZrO}_{3}$ and $\mathrm{PbTiO}_{3}$ is studied extensively to gain insight regarding the nature of their dramatically different temperature trends. Finally, a model for the thermal conductivity of PZT as a function of composition $(\kappa(x))$ is developed and presented.

- Chapter 5: Demonstration of a phonon thermal switch is presented. The device operates by modulating the ferroelastic domain structure in a bilayer PZT thin film via the application of an electric field, ultimately affecting the phonon scattering rates within the material. Using two different types of electrical probing stations integrated 
into our TDTR system, the thermal conductivity of the devices was measured both at room temperature and over temperature as a function of applied electric field.

- Chapter 6: The core concepts and outcomes of this dissertation are summarized and their importance regarding both the basic science and aforementioned applications associated with dynamic thermal transport are discussed. Potential directions for future research are proposed. 


\section{Chapter 2}

\section{Theory \& Concepts}

This chapter is intended to provide the reader with the necessary background regarding the core concepts contained in this dissertation; namely the idea of phonons as thermal carriers, domains and domain boundaries in ferroelectric materials, and the interaction between phonons and domain boundaries studied in the literature to date.

\subsection{Phonons}

In materials that are characterized as either semiconductors or insulators, phonons are the primary thermal energy carriers. A phonon is defined as a quantized lattice vibration; that is, an elastic wave that can only exist as discrete energies, as ascribed by the crystalline structure of the vibrational medium. The propagation of these lattice vibrations through a material is what constitutes the thermal heat flux and the ease with which this heat flux can travel through a material is related to the thermal conductivity, $\kappa$.

A convenient construct for describing the thermal conductivity of a material based on the properties of the energy carriers comes from the kinetic theory of gases [36]

$$
\kappa=\frac{1}{3} C_{v} v \lambda=\frac{1}{3} C_{v} v^{2} \tau
$$


where $C_{v}$ is the volumetric heat capacity of the material, $v$ is the velocity of the energy carriers, $\lambda$ is the carrier mean free path $(\lambda=v \tau)$ and $\tau$ is the relaxation time between carrier collisions. The volumetric heat capacity $\left(C_{v}\right)$ is the temperature-derivative of the internal energy, $U,\left(C_{v}=\partial U / \partial T\right)$, incorporating information about what discrete energy states exist in a given material, the density of those states around a given energy and the probability that those states are occupied at a given $T$ based on the Bose-Einstein distribution. The velocity $(v)$ specifies the speed with which the phonons propagate through the material, where larger $v$ results in a larger value of $\kappa$. The mean free path $(\lambda)$ represents the average length a phonon travels between scattering events that impede their propagation through the material. It can also be expressed in terms of the velocity of the phonons and the time between scattering events ( $\tau$, referred to as the scattering or relaxation time).

$$
\lambda=v \tau
$$

In general, the smaller the value of $\lambda$ or $\tau$, the lower the thermal conductivity of the material due to the increased rate of collisions that impede phonon transport. One means of accomplishing this is through material nanostructuring [29, 30, 37, 38]; that is, the introduction of features and/or anomalies in the material on the nanoscale that make phonons scatter more frequently than they otherwise would. Much of my prior research leading up to this dissertation work has focused on studying the impact of nanostructuring on the thermal conductivity of several complex oxide materials. The prior projects include a lead role studying nanograined strontium titanate $\left(\mathrm{SrTiO}_{3},[4]\right)$, a supporting role in studying nanograined barium titanate $\left(\mathrm{BaTiO}_{3},[5]\right)$ and another lead role studying strontium niobate $\left(\mathrm{Sr}_{2} \mathrm{Nb}_{2} \mathrm{O}_{7},[9]\right)$. One of, if not the most important conclusions from this collection of work was that a relatively broad spectrum of phonon energies, and thus mean free paths contribute to the thermal conductivity of complex oxide materials, contrary to what had been assumed previously that phonon conduction in complex oxides was governed by a single mean free path (i.e., a gray medium). The following sections will describe the highlights of this previous research, as well as the application of the concepts learned to phonon 
scattering at ferroelastic domain walls.

\subsection{Phonon Scattering in Complex Oxides}

\subsubsection{Incoherent Interfaces: Nano-grained $\mathrm{ABO}_{3}$ Perovskites}

One of the first questions that I addressed in my graduate studies concerned the nature of phonon transport in perovskite (chemical formula $A B \mathrm{O}_{3}$ ) oxides. In two separate projects, we measured the thermal conductivity, $\kappa$, of nanograined strontium titanate (ng$\left.\mathrm{SrTiO}_{3}\right)$ [4] and nanograined barium titanate $\left(\mathrm{ng}-\mathrm{BaTiO}_{3}\right)$ [5] thin films with varied average grain sizes that were fabricated through chemical solution deposition (CSD) at Sandia National Laboratories. CSD is an inexpensive and scalable technique for producing a variety of thin film materials over large areas with a high degree of repeatability [39-41]. By varying the temperature and duration of a post-processing anneal, we were able to control the average grain size of the thin films. Grain sizes in the $\mathrm{ng}-\mathrm{SrTiO}_{3}$ sample set ranged from 28-88 nm, while the ng- $\mathrm{BaTiO}_{3}$ samples ranged from 36-63 nm.

The thermal conductivities of the two different oxides as a function of average grain size are plotted in Figure 2.1. In both materials, the thermal conductivity decreases as the average grain size decreases. This is an important result which highlights the fact that there exists a spectrum of phonon mean free paths which are present in these complex oxide materials at room temperature. In previously published works, the thermal conductivities of $\mathrm{SrTiO}_{3}$ and $\mathrm{BaTiO}_{3}$ had been modeled [42] using a gray-medium approximation [43] in which a singular phonon mean free path is assumed on the order of $2-5 \mathrm{~nm}$. The measured data in our published works clearly contradicts that this could be the case, for if the mean free paths were indeed limited to a value between $2-5 \mathrm{~nm}$, we would see minimal dependence of the thermal conductivity on the average grain size (i.e., the data would be flat).

To support this point, each publication included a model for the thermal conductivity using expressions from kinetic theory to highlight the spectral nature of phonon transport 

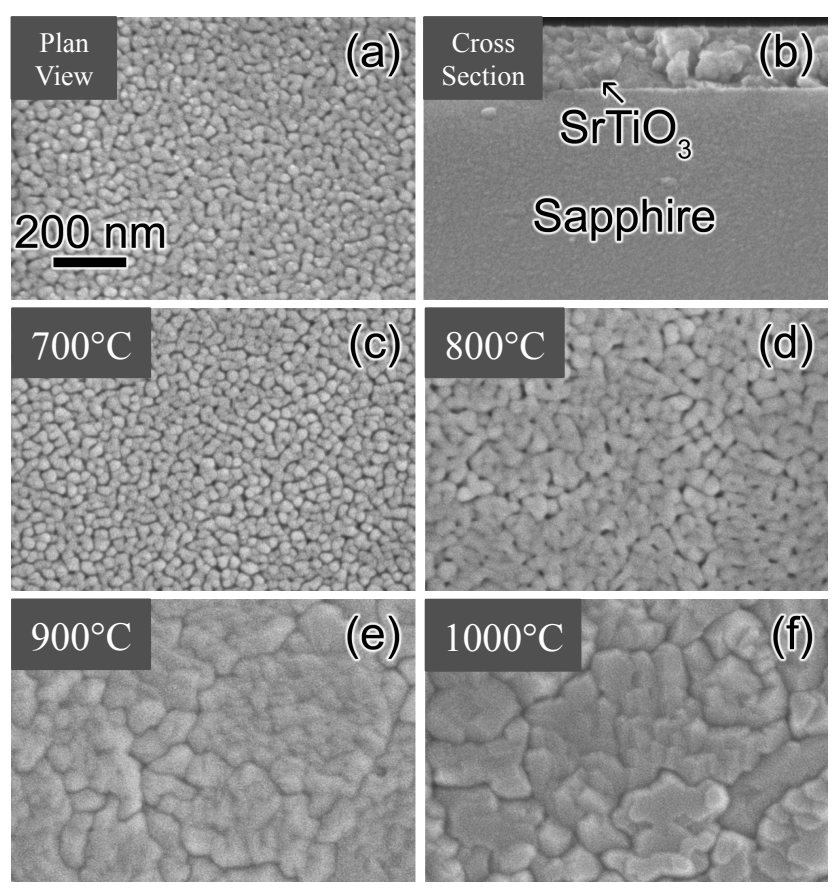

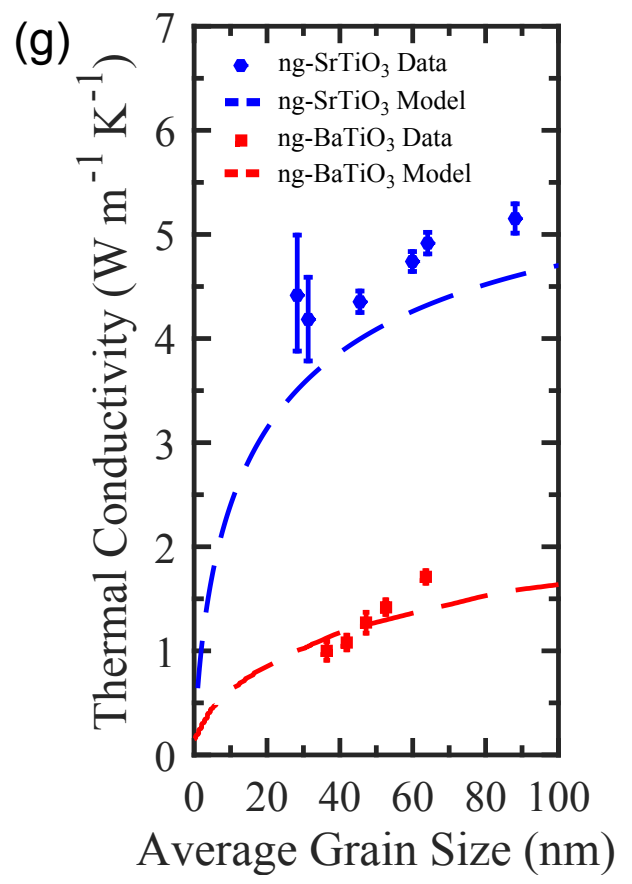

Figure 2.1: (a) Plan and (b) cross sectional SEM micrographs of as crystallized $\mathrm{SrTiO}_{3}$ films on (0001) sapphire. Plan view images of films that were post annealed at (c) 700, (d) 800, (e) 900, and (f) $1000^{\circ} \mathrm{C}$ demonstrate the degree to which post crystallization anneals impact the film microstructures. [4] (g) Thermal conductivity of nano-grained $\mathrm{SrTiO}_{3}\left(\mathrm{ng}-\mathrm{SrTiO}_{3}\right)$ and nano-grained $\mathrm{BaTiO}_{3}$ $\left(\right.$ ng- $\left.\mathrm{BaTiO}_{3}\right)$ as a function of average grain size at room temperature. Also plotted are models for the thermal conductivity as a function of grain size derived from kinetic theory. [4, 5]

in the nano grained samples. The models are based on the following extended version of Eq. 2.3.

$$
\kappa=\frac{1}{3} \sum_{j} C_{v, j} v_{j}^{2} \tau_{j}=\frac{1}{6 \pi^{2}} \sum_{j} \int_{k} \hbar \omega_{j} \frac{\partial f_{\mathrm{BE}}}{\partial T} v_{j}^{2} \tau_{j} k^{2} d k
$$

where $j$ is the phonon polarization index, $\omega$ is the angular frequency, $\partial f_{\mathrm{BE}} / \partial T$ is the temperature derivative of the Bose-Einstein distribution function, and $k$ is the phonon wavevector. Speaking to my $\mathrm{SrTiO}_{3}$ work, the experimentally-determined dispersion in the [100] direction from Cowley[44] was used by fitting each of the 15 phonon branches with fourth-order polynomials. The total scattering time, $\tau_{j}$, as the combination of the scattering times due to both anharmonic phonon-phonon $\left(\tau_{\mathrm{a}}\right)$, and phonon-grain boundary $\left(\tau_{\mathrm{gb}}\right)$, and 
phonon-film boundary $\left(\tau_{\mathrm{fb}}\right)$ scattering. The total scattering rate is then determined through Matthiessen's rule [36], given as

$$
\begin{aligned}
\tau_{j} & =\left[\frac{1}{\tau_{\mathrm{a}}}+\frac{1}{\tau_{\mathrm{gb}}}+\frac{1}{\tau_{\mathrm{fb}}}\right]^{-1} \\
& =\left[B T \omega_{j}^{2} \exp \left(-\frac{C}{T}\right)+\frac{v_{j}}{d_{\mathrm{avg}}}+\frac{v_{j}}{170 \mathrm{~nm}}\right]^{-1}
\end{aligned}
$$

where $d_{\text {avg }}$ is the average grain size and the last term represents scattering due to the film thickness $(170 \mathrm{~nm})$. The constants $B$ and $C$ in the expression for phonon-phonon scattering were determined by fitting Eq. 2.3 to temperature-dependent experimental data for bulk $\mathrm{SrTiO}_{3}[14,45]$. As $d_{\mathrm{avg}}$ decreases, the relative contribution of $\tau_{\mathrm{gb}}$ to the total scattering time increases, causing a reduction in the thermal conductivity of the associated films.

The principle conclusion from these two projects on nano grained thin films was that there is a fair amount of tunability within the complex oxide material family to engineer thermal transport properties via nano structuring. As interest in complex oxides as potential thermoelectric materials spiked due to their attractive electrical properties [46, 47], the assumption was that the comparatively low thermal conductivities of these oxides [22] were due to intrinsically short mean free paths of less than $10 \mathrm{~nm}$ at room temperature. Because of this, the belief was that size effects with characteristic lengths longer than $10 \mathrm{~nm}$ would have no impact on the thermal conductivity and that the benefits of nano structuring to lower thermal conductivities in other materials [38] would not apply to these oxides. These works on nano grained $\mathrm{SrTiO}_{3}$ and $\mathrm{BaTiO}_{3}$ showed that these assumptions are false and that there is an entire spectrum of phonon mean free paths in these materials at room temperature, affording considerable flexibility in tuning the thermal conductivities of these materials via nano structuring.

\subsubsection{Coherent Interfaces: $\mathrm{Sr}_{2} \mathrm{Nb}_{2} \mathrm{O}_{7}$}

Additionally, several works on phonon scattering in transition metal oxides have focused on the impact of coherent interfaces within these materials on thermal transport. 
Where the works on nano grained $\mathrm{SrTiO}_{3}$ and $\mathrm{BaTiO}_{3}$ focused on the impact of incoherent grain boundaries, research on naturally-layered strontium niobate $\left(\mathrm{Sr}_{2} \mathrm{Nb}_{2} \mathrm{O}_{7}\right)[9]$ demonstrated that changes in local strain and bonding where crystalline coherence is maintained can have a dramatic effect on phonon transport within these materials. The importance of this is the fact that the ability to create phonon scattering sites while maintaining the periodicity of the crystal lattice is an important step towards the electron-crystal/phonon-glass material criteria that is the ultimate goal for high-efficiency thermoelectrics.

$\mathrm{Sr}_{2} \mathrm{Nb}_{2} \mathrm{O}_{7}$ is an orthorhombic perovskite phase (space group \#36-Cmc21) composed of octahedrally coordinated niobium and 12-coordinated strontium, separated by a $\mathrm{SrO}$ double layer in the cross-plane direction [48] (in the standard setting, the direction perpendicular to the layers and referred to as the $b$-axis direction). Figure 2.2 (b) shows a transmission electron microscopy (TEM) image illustrating the layered crystal structure of $\mathrm{Sr}_{2} \mathrm{Nb}_{2} \mathrm{O}_{7}$ with the $b$-axis normal to the $\mathrm{SrTiO}_{3}$ substrate.

Figure 2.2(a) is a plot of the thermal conductivity of two La-doped (5\%) strontium niobate samples $\left(\mathrm{Sr}_{1.9} \mathrm{La}_{0.1} \mathrm{Nb}_{2} \mathrm{O}_{7}\right)$ with different film thicknesses $(130 \mathrm{~nm}$ in blue, $800 \mathrm{~nm}$ in red, respectively) as a function of temperature from 80 to $500 \mathrm{~K}$, along with literature data of several materials including those with weakly-bonded, naturally-layered structures as well as amorphous $\mathrm{SiO}_{2}\left(a-\mathrm{SiO}_{2}\right)$. Figure 2.2 $\mathrm{b}$ b) is a high angle annular dark field scanning transmission electron microscopy (HAADF-STEM) image illustrating the layered crystal structure of strontium niobate. Like the other layered-structures shown in Fig. 2.2 (a), the thermal conductivities of both $\mathrm{Sr}_{1.9} \mathrm{La}_{0.1} \mathrm{Nb}_{2} \mathrm{O}_{7-\delta}$ films are less than $a-\mathrm{SiO}_{2}$ across the temperature range, demonstrating the strong role that phonon scattering at weakly bonded layers can have on the thermal conductivity of crystalline materials. Additionally, our data show good agreement with cross-plane values for highly-textured, bulk single-crystalline $\mathrm{Sr}_{5} \mathrm{Nb}_{5} \mathrm{O}_{17}$ samples [7] (exclusively composed of $n=5$ material in the $\mathrm{Sr}_{n} \mathrm{Nb}_{n} \mathrm{O}_{3 n+2}$ homologous series) measured via a thermocouple-based, steady state technique. Lastly, the thermal conductivity of $\mathrm{Sr}_{1.9} \mathrm{La}_{0.1} \mathrm{Nb}_{2} \mathrm{O}_{7}$ is larger than that of other layered material systems, including $\mathrm{CsBiNb}_{2} \mathrm{O}_{7}([49])$ and $\mathrm{WSe}_{2}([\overline{8}])$. This is consistent with the fact that 

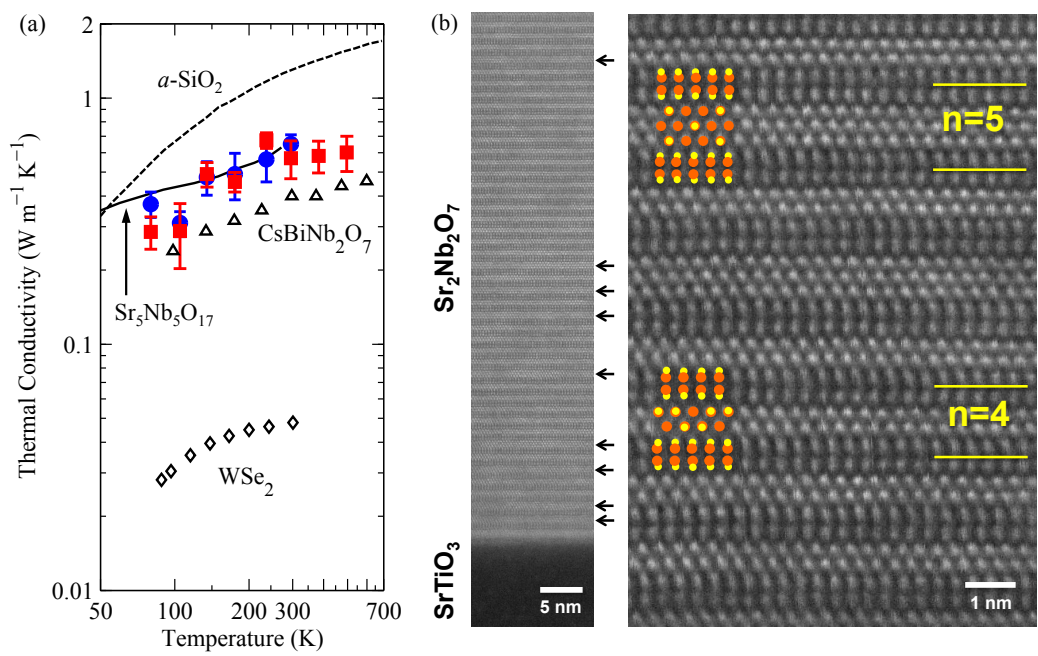

Figure 2.2: (a) Thermal conductivity versus temperature for two $5 \% \mathrm{La}(\mathrm{x}=0.1) \mathrm{Sr}_{2-x} \mathrm{La}_{x} \mathrm{Nb}_{2} \mathrm{O}_{7-\delta}$ samples of different thicknesses; $130 \mathrm{~nm}$ (blue circles) and $800 \mathrm{~nm}$ (red squares). Literature data for amorphous $\mathrm{SiO}_{2}$ (dashed line, [6]), bulk $\mathrm{Sr}_{5} \mathrm{Nb}_{5} \mathrm{O}_{17}$ (solid line, [7]), $\mathrm{CsBiNb}_{2} \mathrm{O}_{7}$ (triangles, citecahill2010aa) and $\mathrm{WSe}_{2}$ (diamonds, [8]) are included for comparison. (b) Atomic-resolution HAADF-STEM images illustrating the layered crystal structure of strontium niobate. Arrows along the left image indicate the position of defects in the layering sequence. The image on the right shows a higher magnification of a section of the grain overlaid with $\langle 101\rangle$ projections of the $\mathrm{Sr}$ (yellow) and $\mathrm{Nb}$ (orange) atom positions in the $\mathrm{Sr}_{2} \mathrm{Nb}_{2} \mathrm{O}_{7}\left(n=4 \mathrm{NbO}_{6}\right.$ octahedra) and $\mathrm{Sr}_{5} \mathrm{Nb}_{5} \mathrm{O}_{17}(n=5$ $\mathrm{NbO}_{6}$ octahedra) crystal structures. [9]

the cross-plane (b-axis) longitudinal speed of sound $\left(v_{L}\right)$ in $\mathrm{Sr}_{2} \mathrm{Nb}_{2} \mathrm{O}_{7}$ is $5192 \mathrm{~m} / \mathrm{s}([50])$, which is larger than the similarly-directed sound velocities of the aforementioned materials ( $v_{L}$ equal to $3350 \mathrm{~m} / \mathrm{s}$ from [49] and $1650 \mathrm{~m} / \mathrm{s}$ from [8], respectively). The differences in the cross-plane sound velocities of these materials can be attributed to the strength of the bonding between the layers; the bonds between $\mathrm{WSe}_{2}$ layers being the weakest while those between perovskite-slabs in $\mathrm{Sr}_{1.9} \mathrm{La}_{0.1} \mathrm{Nb}_{2} \mathrm{O}_{7-\delta}$ being the strongest. The weaker bonds lead to stronger phonon scattering at the layer interfaces, leading to lower thermal conductivities.

Figure 2.3 shows our La-doped films of different thicknesses along with two versions of the minimum-limit model for thermal conductivity to provide context. Assuming an 


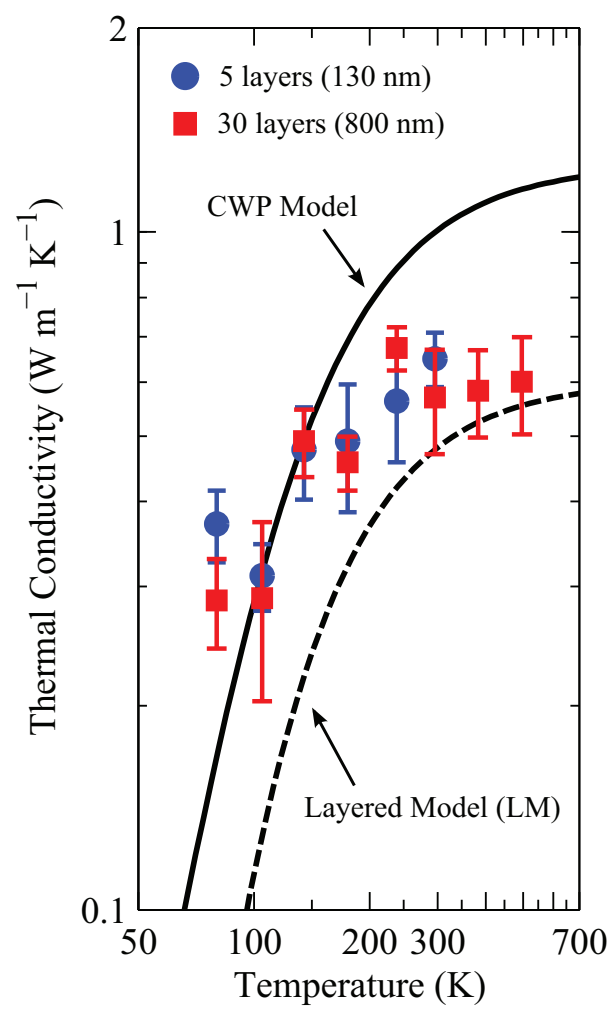

Figure 2.3: Thermal conductivity versus temperature for two 5\% La $(x=0.1) \mathrm{Sr}_{2-x} \mathrm{La}_{x} \mathrm{Nb}_{2} \mathrm{O}_{7-\delta}$ samples of different thicknesses plotted along with two models for the minimum thermal conductivity of a solid; the Cahill-Watson-Pohl (CWP) model [10] and a modified version of the CWP that includes the effects of scattering between weakly-bonded layers (dashed line, this work).

isotropic Debye solid, the expression for the minimum phonon thermal conductivity is

$$
\kappa_{\min }=\frac{\hbar^{2}}{6 \pi^{2} k_{B} T^{2}} \sum_{j} \int_{0}^{\omega_{c, j}} \tau_{\min , j} \frac{\omega^{4}}{v_{j}} \frac{\exp \left[\frac{\hbar \omega}{k_{B} T}\right]}{\left(\exp \left[\frac{\hbar \omega}{k_{B} T}\right]-1\right)^{2}} d \omega
$$

where the summation is over the three acoustic phonon modes (one longitudinal, two transverse), $\omega_{c, j}$ is the cutoff frequency and $\tau_{\min , j}$ is the minimum scattering time. To evaluate Eq. 2.5 for our material system, we use $v_{\mathrm{L}}=5192 \mathrm{~m} / \mathrm{s}$ from Ref. [50] and calculate $v_{\mathrm{T}}=$ $v_{\mathrm{L}} \sqrt{\left(c_{55} / c_{22}\right)}$ using literature values for the elastic constants [51] of $\mathrm{Sr}_{2} \mathrm{Nb}_{2} \mathrm{O}_{7}$. Additionally, we use $n=72.993 \mathrm{~nm}^{-3}$ for the atomic density [52] of $\mathrm{Sr}_{2} \mathrm{Nb}_{2} \mathrm{O}_{7}$ in calculating the cutoff frequencies $\left(\omega_{c, j}=v_{j}\left(6 \pi^{2} n\right)^{1 / 3}\right)$.

The solid line in Figure 2.3 is the Cahill-Watson-Pohl (CWP) model [10] and the dashed 
line is a modified version of the CWP that we refer to as the Layered Model (LM) [53, 54]. Our LM calculations include the effects of scattering between weakly bonded layers. Like the work in [49] and [8], our data lie below the expected minimum thermal conductivity predicted by the CWP. The difference between the two models lies in the definition of $\tau_{\min }$; the CWP model defines the minimum scattering time to be one-half the period of oscillation between adjacent atoms in a given material, $\tau_{\min , j, \mathrm{CWP}}=\pi / \omega$. The layered model incorporates an additional term via Matthiessen's rule [36] that accounts for scattering at the interface between two different layers. The minimum scattering time thus takes the form

$$
\tau_{\mathrm{min}, j, \mathrm{LM}}=\left(\frac{\omega}{\pi}+v_{j} \frac{\pi^{5} n}{\omega^{2}}\left(v_{j}-\frac{\omega d}{\pi}\right)^{2}\right)^{-1},
$$

where the first term is the scattering within the layers and the second is the scattering between layers, which is dependent on the separation distance, $d$. In the case of small $d$ and weak bonding between layers (resulting in lower Debye cutoff frequencies), the difference between the modal sound speed and inter-layer velocities is maximized, resulting in scattering times that approach the inter-atomic scattering times assumed in the CWP. The result is a reduction in the predicted minimum thermal conductivity due to the combined contributions of these separate scattering mechanisms. As we can see in Figure 2.3, the LM lies below our measured data suggesting that the incorporation of inter-layer scattering successfully establishes a new theoretical minimum thermal conductivity that is applicable to similarly layered structures.

\subsection{Domains \& Domain Walls/Boundaries}

In addition to the inter-layer scattering effects observed in $\mathrm{Sr}_{2} \mathrm{Nb}_{2} \mathrm{O}_{7}$ crystals, ferroelastic domain walls have been identified as an additional type of coherent interface that can scatter phonons both at cryogenic [13] and room temperatures [16]. Before reviewing this work, however, we begin with an overview of domains and domain walls in ferroelectric materials. 
Ferroelectric materials are those that possess a spontaneous polarization vector in the absence of an electric field. [21, 55] Additionally, the direction of this polarization vector may be switched via the application of an external electric field. These polarization vectors are due to the distortion of the unit cell of the crystal in some fashion, such as the displacement of the $B$-site atom in $A B \mathrm{O}_{3}$ perovskite oxides. Figure 2.4(a) illustrates this type of distortion along the $c$-axis of tetragonal PZT due to the displacement of the atom (either Ti or $\mathrm{Zr}$ ) from the center of the unit cell. Most ferroelectric materials have a higher-symmetry, paraelectric phase at elevated temperatures where the distortion of the unit cell, and thus the spontaneous polarization disappears. The temperature at which the material goes from ferroelectric to paraelectric (or vice-versa) is called the Curie temperature $\left(T_{\mathrm{C}}\right)$. [21] Ferroelectric materials harbor the complex interplay of thermodynamic, electric and mechanical properties of materials, making them a particularly fascinating area of research towards the development of new functional properties. [56]

While a chunk of ferroelectric material may exhibit a spontaneous polarization in a particular direction on a macroscopic scale, the polarization vector field throughout the crystal is usually non-uniform. In reality, the macroscopically-measured spontaneous polarization is a superposition of all the individual polarizations of various sub-regions within the crystal as a whole. A ferroelectric domain is defined as a region of a crystal where the electric polarization points in the same direction, usually due to a commensurate distortion along a specific crystallographic direction within the unit cell. [55] The boundary between two regions of a crystal with different polarizations is called a domain wall and can have a variety of different properties depending upon the relative orientation of the polarization vectors in the adjacent crystal regions. A domain wall is called ferroelectric when these polarization vectors are oriented $180^{\circ}$ with one another. Alternatively, a domain wall is ferroelastic when these polarization vectors are at any angle other than $180^{\circ}$ (e.g., $90^{\circ}, 71^{\circ}, 109^{\circ}$, etc.), resulting in a large degree of strain that exists to mitigate the difference in local strain between the adjacent regions with different distortion vectors. Figure 2.4(b) highlights the domains and their polarization vectors (large yellow arrows) as well as the ferroelectric 
(a)

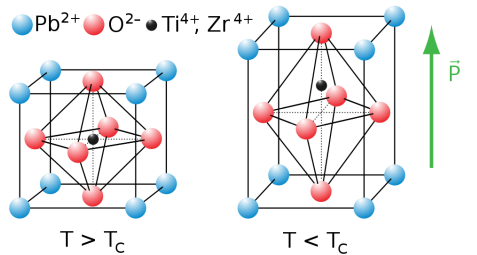

(c)

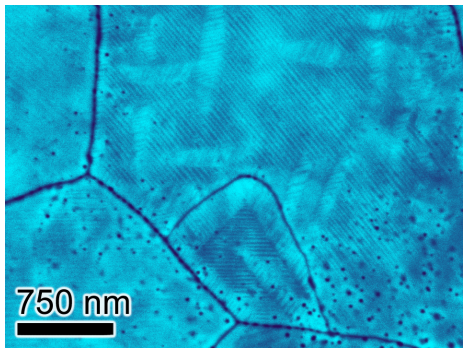

(b)

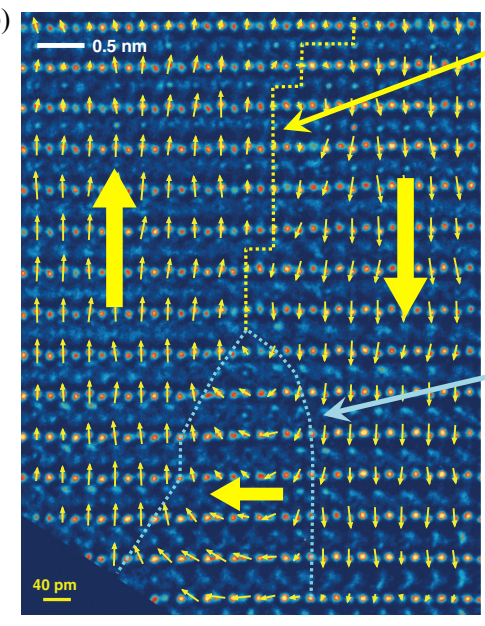

$\underline{180^{\circ} \text { Ferroelectric Domain Wall: }}$

Nominally no strain

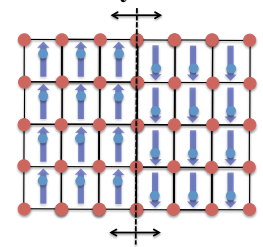

90 Ferroelastic Domain Wall:

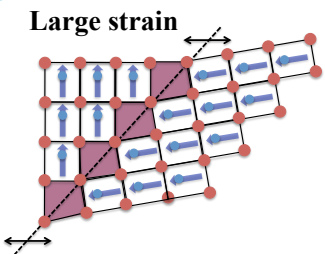

Figure 2.4: (a) Diagram of the tetragonal PZT unit cell, illustrating the distortion along the $c$-axis at temperatures below the Curie temperature $\left(\mathrm{T}_{\mathrm{C}}\right)$ that results in a spontaneous polarization vector, $\overrightarrow{\mathrm{P}}$. (b) Atomic-resolution, aberration-corrected transmission electron microscopy (TEM) image illustrating the displacement of the central B-site atom, and thus the local spontaneous polarization vectors in a $\mathrm{PbZr}_{0.2} \mathrm{Ti}_{0.8} \mathrm{O}_{3}$ thin film [11]. The large yellow arrows denote the average polarization direction in each region (domain) of the crystal separated by the dashed lines. These dashed lines correspond to different types of domain walls separating these domain regions, including ferroelectric walls (yellow dashes) and ferroelastic walls (blue dashes). (c) Plan-view channeling-contrast scanning electron microscopy (CC-SEM) image of a bilayer PZT thin film studied in this dissertation, depicting the complex arrangement of ultra-fine nano-domains bounded by ferroelastic domain walls. [12]

and ferroelastic domain boundaries (yellow dashed and blue dashed lines, respectively) in a high-resolution transmission electron microscopy (TEM) image of $\mathrm{PbZr}_{0.2} \mathrm{Ti}_{0.8} \mathrm{O}_{3}$ from [11]. As the crystal experiences external stimuli in the form of an applied stress or an electric field, the domain structure within the crystal changes to minimize the local energy landscape leading to both the creation/annihilation as well as the movement of domain walls. [55]

In practice, the arrangement of domains and domain walls within a ferroelectric crystal can be quite chaotic, driven primarily by the electrical and mechanical boundary conditions and to a lesser degree by crystalline defects (vacancies, dislocations, etc.). Figure 2.4(c) is a channelling-contrast scanning electron microscopy (SEM) image taken of one of the bilayer PZT samples investigated in this work [12]. This image highlights the complexity of the domain structure in these films, even within individual grains where domains and groups of 
domains can create a variety of length-scales over which spontaneous polarizations exist. Domain-engineering in ferroelectric materials is an immensely active area of research with implications on a wide variety of applications [56] as it is the creation, annihilation and reconfiguration of these domains that give ferroelectric materials their remarkable extrinsic properties.

\subsection{Phonon Scattering at Domain Walls/Boundaries}

There have been a few different studies focused on the scattering of phonons at ferroelastic domain walls in the literature. One of the first published works was that of Mante and Volger on barium titanate $\left(\mathrm{BaTiO}_{3}\right)$ single crystals [13]. Figure 2.5. (a) plots the data from [13] of the thermal conductivity of $<100>$-oriented $\mathrm{BaTiO}_{3}$ single crystals. Measurements were made over temperatures ranging from 3 to $50 \mathrm{~K}$ both before and during the application of an $11 \mathrm{kV} \mathrm{cm}^{-1}$ electric field across the $\mathrm{BaTiO}_{3}$ crystal. When the $11 \mathrm{kV} \mathrm{cm}^{-1}$ field was applied across the sample, the thermal conductivity was found to increase by as much as $80 \%$ across much of the temperature range. When the electric field was removed from the sample, the thermal conductivity did not change in value from what was measured with the electric field applied. Only after two weeks while kept under vacuum did the thermal conductivity of the sample change, decreasing in magnitude to values lower than the original virgin state.

The observed increase in thermal conductivity with an applied electric field was attributed to the reduction in the number of ferroelastic domain walls present, as it was already well known that $\mathrm{BaTiO}_{3}$ single crystals can be made single-domain (or close to it) via suitable applied electric field strengths. The reduction in the the domain wall density results in a decrease of the phonon scattering rate with the walls, making it easier for the phonons to flow through the $\mathrm{BaTiO}_{3}$ crystal. It is interesting to note that the change in thermal conductivity between the original and biased states tends to taper down to zero as the temperature of the sample approaches $35 \mathrm{~K}$. While the paper doesn't characterize the 
size of the domains and/or the spacing between domain walls, these trends with temperature suggest that for temperatures less than $35 \mathrm{~K}$, the mean free paths are limited by the domain wall spacings. However, at temperatures above $35 \mathrm{~K}$ where no change in thermal conductivity is observed due to the fact that the phonon mean free path becomes dominated by some other phonon scattering mechanism; mostly likely phonon-phonon scattering.

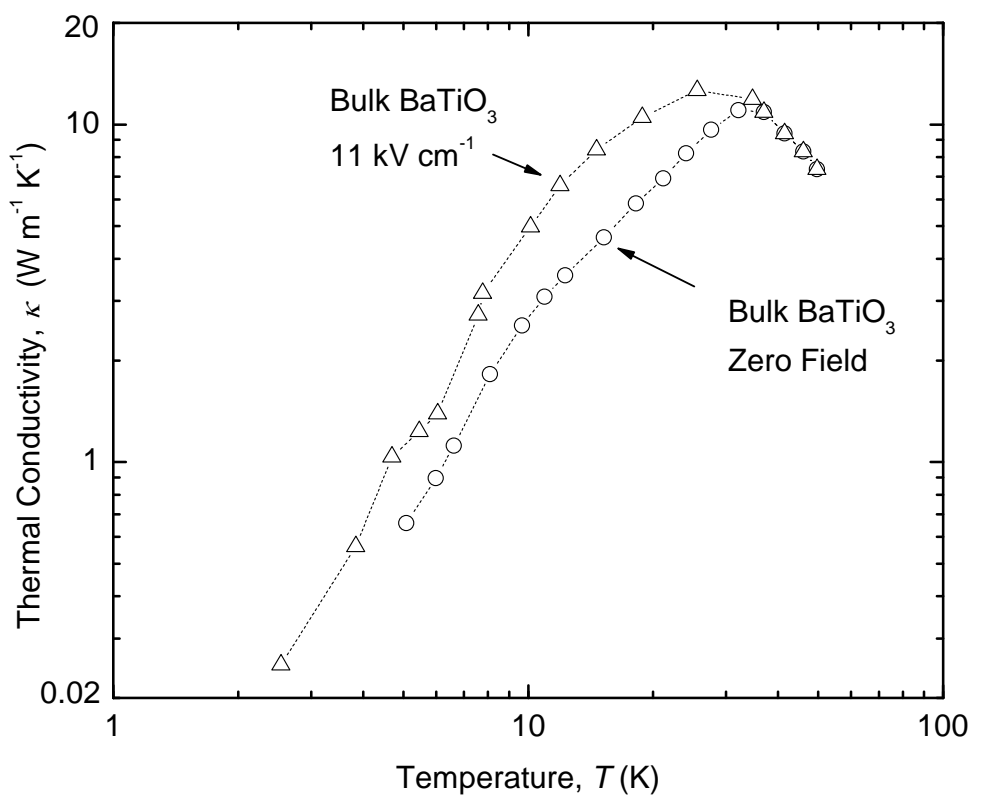

Figure 2.5: Thermal conductivity of $\mathrm{BaTiO}_{3}$ single crystals from 3 - $50 \mathrm{~K}$, measured in [13]. Open circles denote measurements made on the unpoled crystals, while open triangles denote measurements made while the samples were actively poled by an $11 \mathrm{kV} \mathrm{cm}^{-1}$ electric field.

In addition to these results for bulk $\mathrm{BaTiO}_{3}$, ferroelastic domain walls have been shown to scatter phonons at room temperature in bismuth ferrite $\left(\mathrm{BiFeO}_{3}\right)$ thin films, exhibiting a decrease in the effective $\kappa$ as the ferroelastic domain wall density increases [16]. Through the use of substrate vicinality, a series of $\mathrm{BiFeO}_{3}$ thin films with differing numbers of possible domain variants, and therefore different domain wall densities were epitaxially grown via adsorption-controlled molecular-beam epitaxy (MBE) [57, 58]. Figure 2.6 (a) plots the effective thermal conductivity of the $\mathrm{BiFeO}_{3}$ films as a function of temperature, along with 
literature data for bulk $\mathrm{SrTiO}_{3}[14,15]$ and amorphous $\mathrm{SiO}_{2}$ [6]. The domain wall densities present in each of the samples were characterized via piezoresponse force microscopy (PFM), as shown in Figure 2.6(b)(c). Through these PFM maps, the domain wall density was shown to increase with an increasing number of possible domain variants. As the number of domain variants, and thus the domain wall density in the sample increases, the thermal conductivity decreases in magnitude.
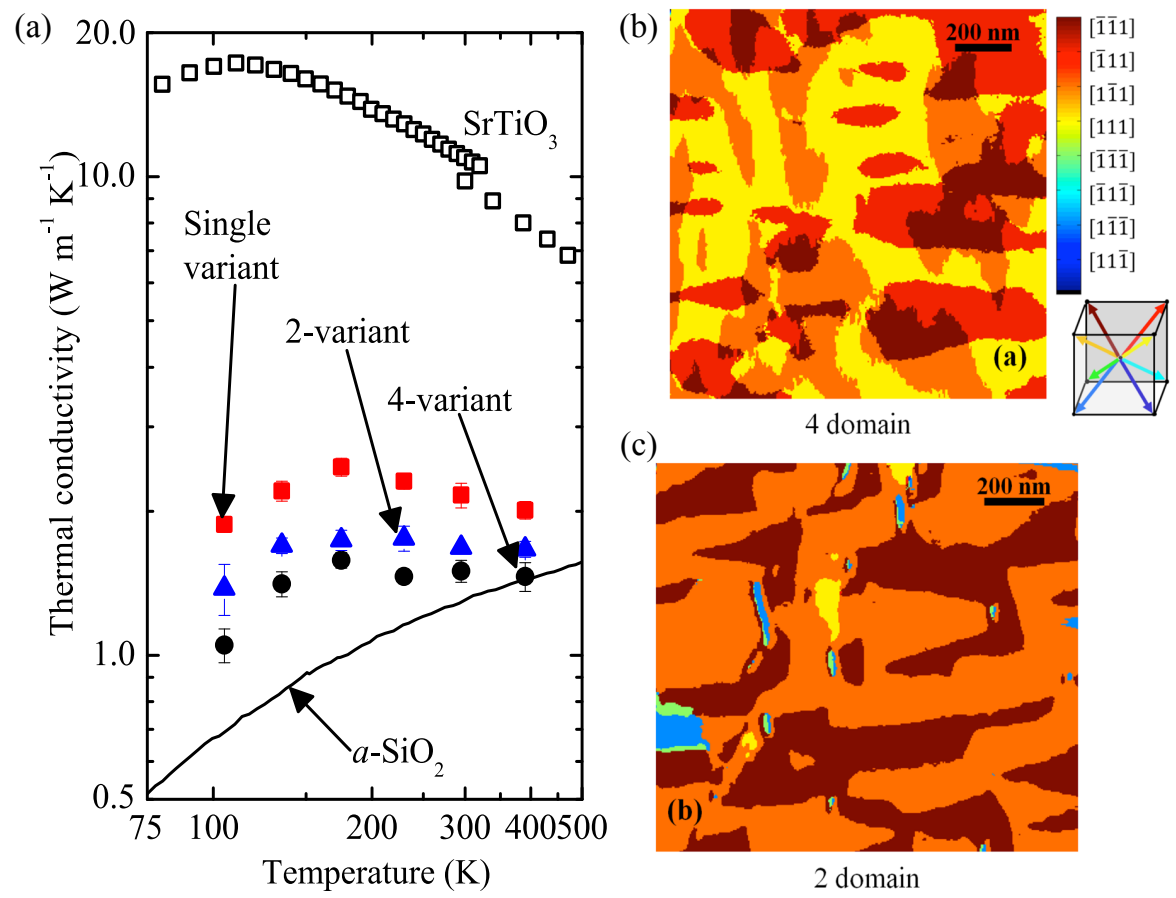

Figure 2.6: (a) Effective thermal conductivities versus temperature for $\mathrm{BiFeO}_{3}$ films with different numbers of domain variants, and thus, different domain wall densities, along with literature data for bulk $\mathrm{SrTiO}_{3}$ (open squares, Refs. [14, 15]) and amorphous $\mathrm{SiO}_{2}$ (solid line, Ref. [6]) for context. $(b, c)$ Plan-view piezoresponse force microscopy (PFM) images illustrating the increase in domain wall density in the 4-domain variant film compared to the 2-domain variant film. The decrease in the thermal conductivity observed in the 4-domain variant case in (a) is attributed to this increase in domain wall density. [16]

While this study utilized separate samples with different domain wall densities, in principle it should be possible to dynamically change the domain wall density in a single sample by applying an electric field across the crystal, as in the $\mathrm{BaTiO}_{3}$ single crystals. One of the challenges with thin film samples like the $\mathrm{BiFeO}_{3}$ is that the ferroelastic domains in these samples are clamped due to strain interactions with the substrate and therefore cannot move 
in response to an applied electric field. [59, 60] In order to capitalize on this phenomena and create a thermal switch, a new material and/or device geometry would be needed to increase the mobility of the ferroelastic domain walls. 


\section{Chapter 3}

\section{Experimental Methods}

\subsection{Time Domain Thermoreflectance (TDTR)}

\subsubsection{System Description}

The thermal transport properties of all the materials and devices measured as part of this work were determined using time domain thermoreflectance (TDTR). TDTR is a noncontact, optical pump-probe technique utilizing ultra-fast laser pulses to measure the diffusion of thermal energy through a material [14]. In practice, the $800 \mathrm{~nm}$ output of a Ti:Sapphire oscillator (Spectra Physics Tsunami, $100 \mathrm{fs}$ pulse width, $80 \mathrm{MHz}$ repetition rate) is split via a polarizing beamsplitter into two pulse-synchronous beams that will serve as the pump and probe beams for the measurement. The pump beam is directed through an electro-optic modulator (EOM, ConOptics $350-160$ driven by a $25 \mathrm{~A}$ linear amplifier) to modulate the pulse-train with an envelope function provided by a function generator. The modulated pump beam creates a periodic heating event at the top surface of the sample as the thermal energy imparted by the pump diffuses through the sample. The probe beam is routed down a mechanical delay stage to provide up to $6 \mathrm{~ns}$ of offset between the arrival of a probe pulse at the sample surface relative to a pump pulse. At a given delay time, the intensity of the reflected probe beam is measured via phase-sensitive detection (lock-in 

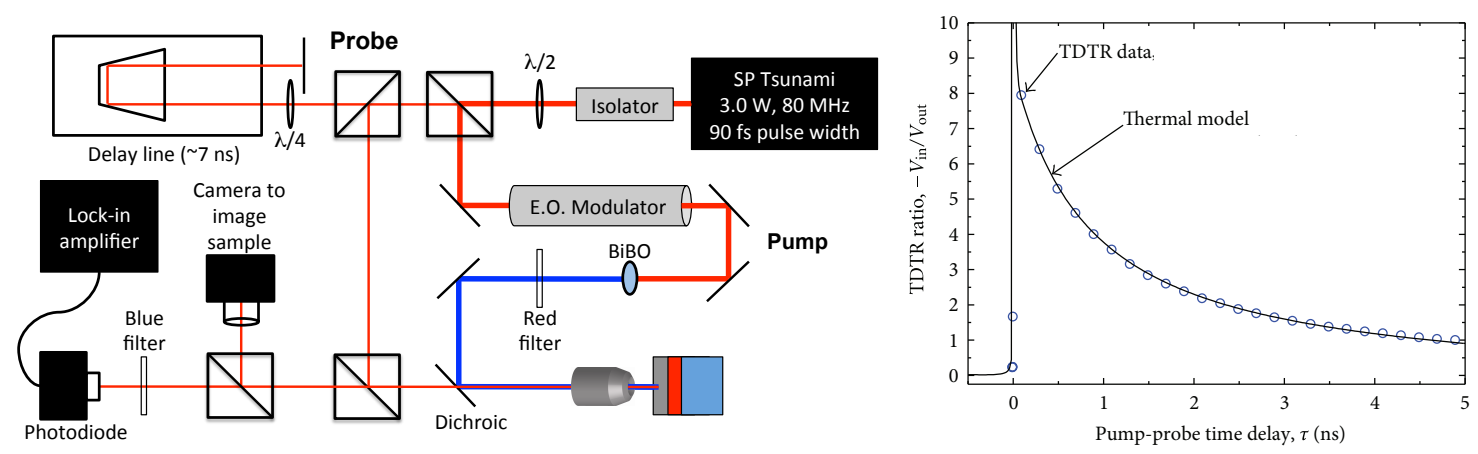

Figure 3.1: Left: Block diagram of the two-color time-domain thermoreflectance (TDTR) system at the University of Virginia. Right: Illustration of the TDTR ratio data being fit to the multilayer thermal model to extract the thermal transport parameters of interest for a given sample. [17]

amplification; Stanford Research Systems SRS 844 and Zurich Instruments HF2LI) at the pump beam modulation frequency due to the change in thermoreflectance of the sample surface. By incrementally elongating the path length that the probe must travel before arriving at the sample, the decay of the sample surface temperature over time can be recorded to monitor how heat diffuses through the sample stack. Figure 3.1(a) depicts a block diagram of the TDTR system, illustrating the key features of the system and the different beam paths traveled by the pump and probe. Figure 3.1 (b) plots a representative set of TDTR ratio data that can then be fit to a given model to extract the transport parameters of interest from the measurement. [17]

An important aspect of the system is the means by which the pump beam is isolated from the probe beam to avoid signal leakage into the detection system. The two methods that we employ on our TDTR systems are the two-color and two-tint approaches; both of which are based on providing isolation through wavelength separation. The two-color method utilizes a bismuth triborate $\left(\mathrm{BiB}_{3} \mathrm{O}_{6}\right)$ crystal to generate $400 \mathrm{~nm}$ (blue) light from our $800 \mathrm{~nm}$ pulse-train via second harmonic generation (SHG) due to the non-linear optical properties of the crystal. By pumping the sample with this $400 \mathrm{~nm}$ light while probing with the unperturbed $800 \mathrm{~nm}$ light, we are able to maximize the signal-to-noise (SNR) of our detection system using fairly inexpensive optical filters. By comparison, the two-tint method employs wavelength separation by splitting the finite bandwidth in wavelength 
space of the femtosecond pulses using extremely sharp high-pass and low-pass optical filters centered around $800 \mathrm{~nm}$. Finally, an additional high-pass filter is used just before the photodetector to further reject the pump wavelengths to maximize the SNR. [61]

To facilitate the measurement, the sample of interest is coated with an approximately 80-100 nm metal film (common metals include aluminum, gold and platinum) to serve as an opto-thermal transducer for the thermoreflectance measurement. When the pump pulse arrives at the metal transducer, the photon flux excites a population of electrons, elevating the electron temperature within the metal. Over a period of about 1-5 ps, the electrons transfer their energy to the crystal lattice via collisions with the atoms through a process referred to as electron-phonon coupling. This energy transfer process elevates the lattice temperature within the metal transducer and creates a thermal gradient that drives the heat flux from the metal transducer to the adjacent layers of the sample. Over the next 100 6000 ps (0.1 - $6 \mathrm{~ns})$, the thermal flux effusing away from the metal film may encounter a variety of scattering processes that impede the procession of the phonons through the material; specific examples include film boundaries, grain boundaries and impurity/defect sites in the lattice. The temperature decay of the metal transducer over this time period is directly related to the degree to which these potential phonon scattering mechanisms impede the phonon flux. Once this decay curve is collected, the TDTR data is fit to a multilayer thermal model [15-17] to extract the thermal parameters of interest, including the thermal conductivity of different layers as well as the thermal boundary conductance between adjacent layers. Additionally, by mounting the samples inside a cryostat with optical access, the TDTR measurements can be performed across a wide range of temperatures.

\subsection{Integration of Electrical Probe Stations}

To perform the in-situ measurement of thermal transport within a thin film material and or device while under an applied DC electric field, it was necessary to integrate an electrical probing station into the TDTR system. This electrical probing station (or probe station, for 
short) enables us to make electrical connections via small, thin film metal electrodes deposited on the samples via the micromanipulation of sharp probe tips. These tips are then wired to standard electrical connectors to facilitate connection of the devices to instrumentation for measurement of the electrical properties. Two separate types of probe stations were integrated into the TDTR system at UVA; a smaller one made by J microTechnology for room temperature measurements exclusively and a larger one made by Janis Research Company, LLC for measurements over temperatures ranging from 80 - $450 \mathrm{~K}$. Each of these probe stations were then connected to a variety of electrical measurement equipment to both bias and characterize the PZT materials and devices. Details concerning these probe stations and the electrical measurement equipment are as follows.

\subsubsection{Room Temperature Probe Station}

The room temperature thermal measurements of the bilayer PZT heterostructures while under applied electrical bias were made using the LMS-2709 Microprobing Station manufactured by $\mathrm{J}$ microTechnology, Inc. The LMS-2709 is a compact probing system that is ideally suited for making accurate measurements in laboratory/research environments where samples are much smaller than the 4-12" diameter wafers commonly processed in industry. The combination of small form-factor and intuitive deign were the driving forces behind the selection of the LMS-2709 over other products made by manufacturers such as Signatone, Micromanipulator and Rucker Kolls. The LMS-2709 has a 2"x2" gold plated chuck that is ported for vacuum hold-down of the samples, mounted on an X-Y travel stage to provide independent movement of the sample relative to the probes.

The system comes with two multi-axis micro-positioners which provide XYZ-control of the probe tips used to make contact to the sample. The positioners use a piece of magnetic material to provide secure attachment to the Ni-plated steel platen that surrounds the chuck. The positioners can be used with either microwave or DC probes, depending on the type of measurement being conducted. For the purposes of our measurement, only DC probes are used as they are able to provide adequate bandwidth for all electrical measure- 




Figure 3.2: Photograph of the LMS-2709 Microprobing Station manufactured by J microTechnology, Inc., prior to the modifications that were made to integrate the unit into the TDTR system.

ments made on the samples (DC-100 kHz). The probe tips used for the electrical measurements are made of Paliney $7^{\mathrm{TM}}$ alloy. This material is ideally suited for low energy, low noise electrical contacts and exhibits a high resistance to corrosion while providing the mechanical properties of beryllium copper. The material is a palladium/silver age-hardened alloy containing 10\% gold and platinum, respectively, that meets the ASTM Standard B540 for electrical contact alloys.

To facilitate thermal measurement via TDTR while under electrical bias, the probe station was integrated into the optical measurement system in a probe-down configuration. In this arrangement, the sample is mounted horizontally on the vacuum chuck to enable electrical contact via the micro-positioners, while the laser for the TDTR measurement is routed vertically to provide perpendicular-incidence to the sample surface. The laser is elevated by approximately 18" using a periscoping kit from Thorlabs, and then directed downward at the sample using an apparatus that is built into the LMS-2709 unit. This apparatus utilized a variety of $30 \mathrm{~mm}$ cage system components to provide accurate alignment 
of the beam along the path, as well as placement of additional components such as the cage-mounted translation stage for $z$-axis adjustment of a 10x Nikon objected to focus the TDTR beams onto the sample.
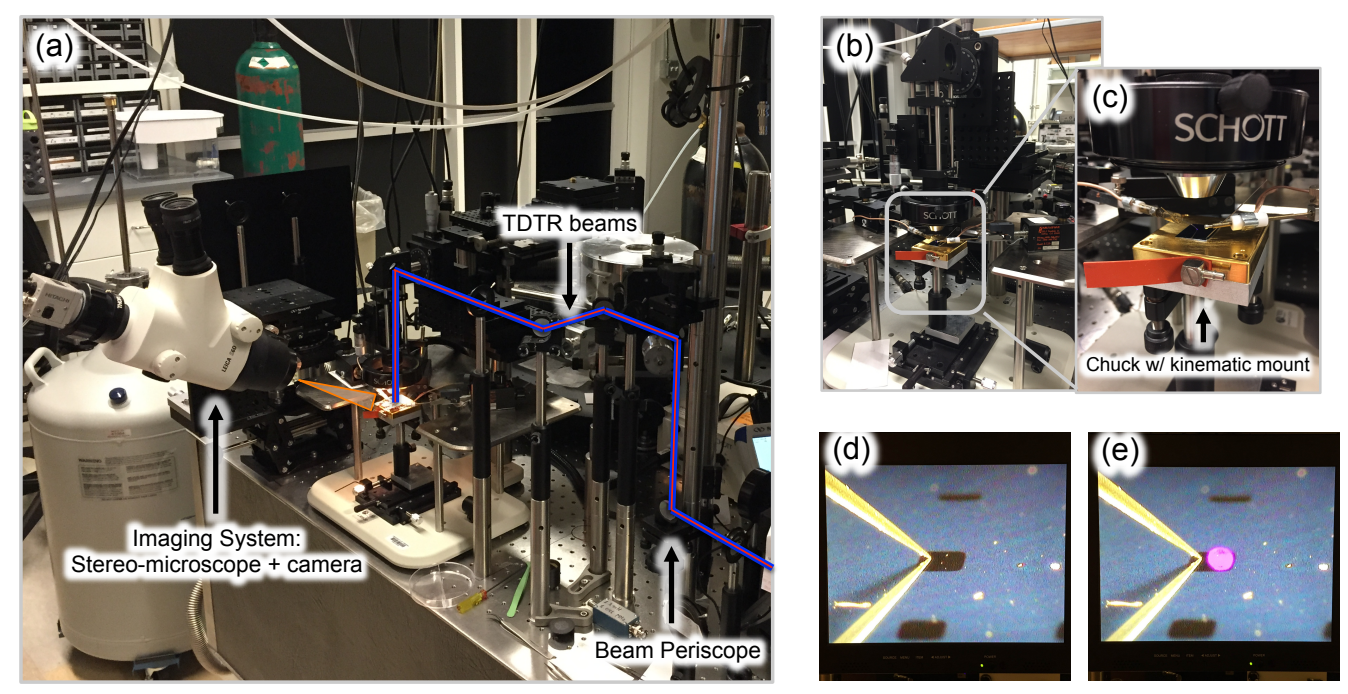

Figure 3.3: (a) Photograph of the modified LMS-2709 probe station integrated into the TDTR system. The path of the coaxial pump and probe beam to the sample sample is highlighted to illustrate the addition optics required to periscope the beam up, as well as direct the beam downward towards the sample mounted on the chuck. Also pictured is the imaging system comprised on a stereo microscope and camera, mounted at an angle to enable accurate placement of the electrical probes onto the top electrodes/thin films. Close-up images of the modified probe station (b) showing the optics included to route the laser beam at normal incidence to the sample surface, as well as the kinematic mount (c) added to the chuck to adjust the planarity of the sample and reflect the beam back to the photo-detector. Images of the sample surface showing an electrical probe tip in contact with a top electrode on a PZT sample, both without (d) and with (e) the laser focused on the electrode.

In order to ensure that the incident beam was indeed perpendicular to the sample and that the reflected TDTR signal was routed to the photo-detector, the LMS-2709 probe station was modified by mounting the vacuum chuck on an kinematic mounting plate. Using this plate, the back-reflection of the TDTR probe laser off the sample surface and back down the beam line could be adjusted to account for any issues in planarity to ensure perfectly normal incidence. Because of the required kinematic mount, the height of the Ni-plated steel platen needed to be raised to compensate for the elevated chuck height. To 
account for this, 1/4-20 taped-holes were added to the LMS-2709 based and 1/2" optical posts were used to bring the platen up to the appropriate level.

Due to probe-down TDTR optics, a custom imaging system was assembled and mounted at an off-normal angle relative to the sample surface so that the electrical probe tips could be placed onto the top electrodes accurately and carefully. This imaging system was comprised of a stereo-microscope with a CCD camera added to the top of the optical column that was then connected to a monitor. By varying the zoom and focus of the microscope, crisp color images of the sample surface could be obtained on the monitor to enable accurate placement of the electrical probe tips on the thin metal electrodes. Figure. 3.3 highlights the placement of this imaging system relative to the room temperature probe station and the images which result.

\section{Use of Probe Station to Measure Electrical Contact Resistance at Metal/Graphene Interfaces}

One of the first projects to use this room temperature probe station was the measurement of the electrical and thermal transport properties of Au-single layer graphene (SLG) contacts with various materials or molecules placed between the Au and SLG. [18] While the electrical and thermal properties were not measured simultaneously like with the thermal devices in this dissertation, this work represented the first demonstration of making high-accuracy measurements with the modified room temperature probe station. This work was performed in collaboration with Dr. Scott G. Walton and Dr. Sandra C. Hernandez in the Plasma Physics Division at the Naval Research Laboratory in Washington, DC. Their expertise in the chemical functionalization and characterization of SLG with various adsorbates via low-energy plasmas was critical to establishing the structure-property relationships proposed in this work.

In short, sheets of SLG grown by CVD and transferred onto $\mathrm{SiO}_{2}$ substrates were chemically functionalized using electron beam generated plasmas. [62-66] Following characterization via X-ray photoelectron spectroscopy (XPS) and micro-Raman spectroscopy, groups 

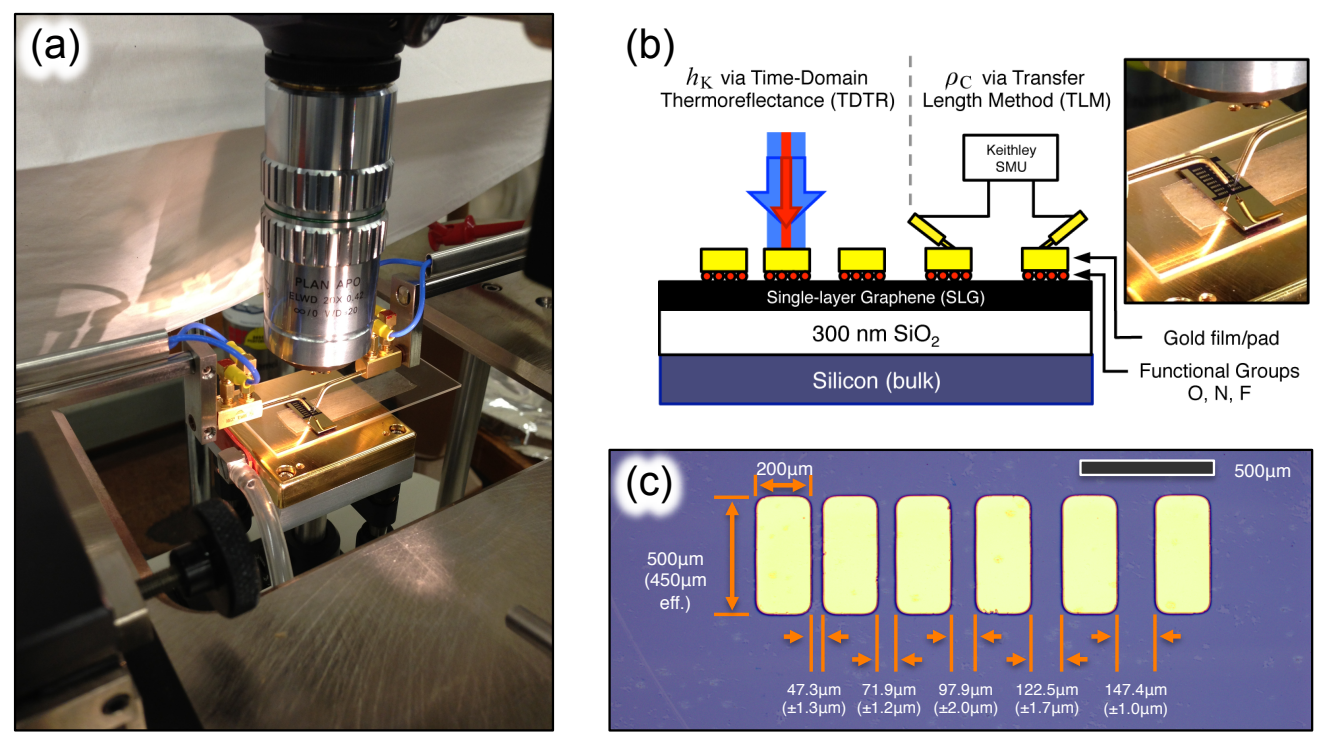

Figure 3.4: (a) Photo of the room temperature probe station making a measurement on one of the functionalized SLG samples from the study. (b) Plan-view diagram of the TLM contact geometry for the in-plane electrical measurements, showing the functional groups underneath the gold pads. (c) Optical plan-view image of the TLM geometry used in this study after fabrication, including the measured distances between pads. [18]

of contacts were patterned via physical masks onto the functionalized SLG to facilitate contact resistivity measurements via the transfer length method (TLM). [20, 67, 68] Using the room temperature probe station, we were able to make accurate in-plane measurements of the pad-to-pad resistances between each adjacent pair of contacts in the TLM structure. Figure 3.4 (a) shows a photo of the room temperature probe station making a measurement on one of the functionalized SLG samples from the study, while Figure 3.4(b) and (c) show a cross-section diagram and plan-view optical image of the TLM contact geometry, respectively.

The room temperature probe station in Figure 3.4(a) was used to measure ten TLM structures for each sample in the study (Au/SLG and Au/Ti/SLG control samples, as well as functionalized SLG samples with three different percent coverages of oxygen and fluorine and one coverage of nitrogen). From these measurements, the width-normalized electrical contact resistance $\left(\rho_{\mathrm{C}}\right)$ of each sample was determined and the results are plotted 


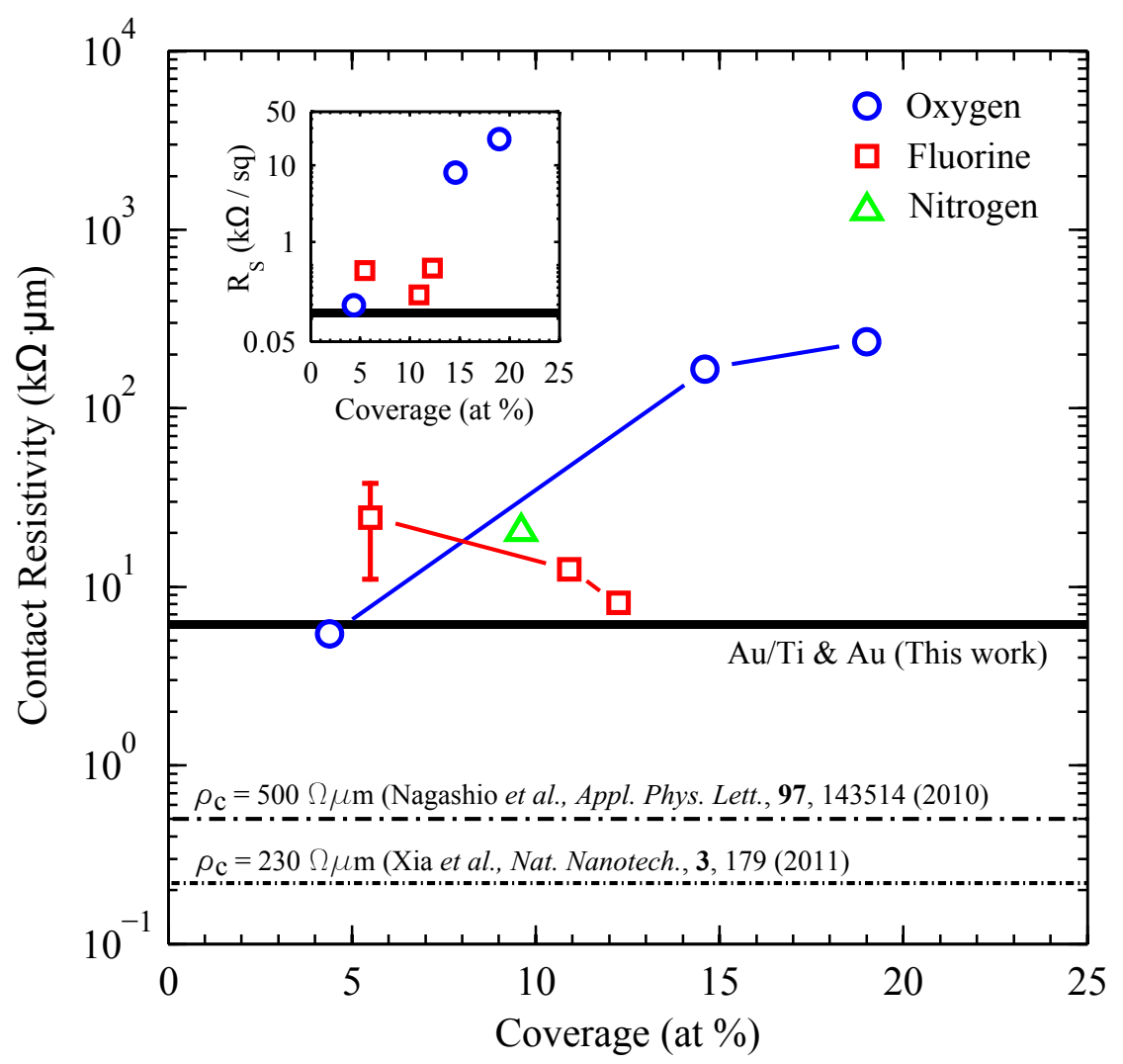

Figure 3.5: Width-normalized contact resistivity $\left(\rho_{\mathrm{C}}\right)$ versus atomic percent (at $\left.\%\right)$ coverage of various adsorbates on single layer graphene. [18] The introduction of oxygen or nitrogen at coverages greater than 5 at $\%$ causes $\rho_{\mathrm{C}}$ to increase dramatically. Conversely, while low coverages of $\approx 5$ at $\%$ fluorine causes an increase in $\rho_{\mathrm{C}}$, coverages greater than 5 at $\%$ cause $\rho_{\mathrm{C}}$ to decrease with increasing coverage. The dashed lines represent contact resistivity values reported for Au/Ti/SLG [19] and palladium/SLG [20], respectively. Inset: Sheet resistance $\left(R_{\mathrm{S}}\right)$ of the graphene between the TLM pads due to doping of the graphene adjacent to the metal contacts that occurred while functionalizing the graphene via physical masks.

in Figure 3.5. The value of $\rho_{\mathrm{C}}$ for both $\mathrm{Au} / \mathrm{SLG}$ and $\mathrm{Au} / \mathrm{Ti} / \mathrm{SLG}$ interfaces is essentially the same. This is consistent with other measurements [19, 69] of the contact resistance between graphene and these metals, and highlights an auxiliary conclusion from this work; the inclusion of a titanium wetting layer between Au and SLG does not improve the electrical (nor thermal) transport across the metal/SLG interface. In the case of oxygenated graphene, $\rho_{\mathrm{C}}$ was found to increase by more than an order of magnitude with increasing coverage. For fluorinated samples, following an initial increase over the non-functionalized case at the lowest coverage, $\rho_{\mathrm{C}}$ actually decreases with increasing coverage and approaches the 
values observed in the non-functionalized contact case (Au/SLG and Au/Ti/SLG). While the exact mechanism behind this observation is quite complex, these findings illustrate how sensitive the transport properties of 2D materials can be to their chemical environment and indicate exciting possibilities using plasma-based chemical functionalization to tailor the thermal and electrical transport properties of metal/2D material contacts.

\subsubsection{Cryogenic Probe Station}
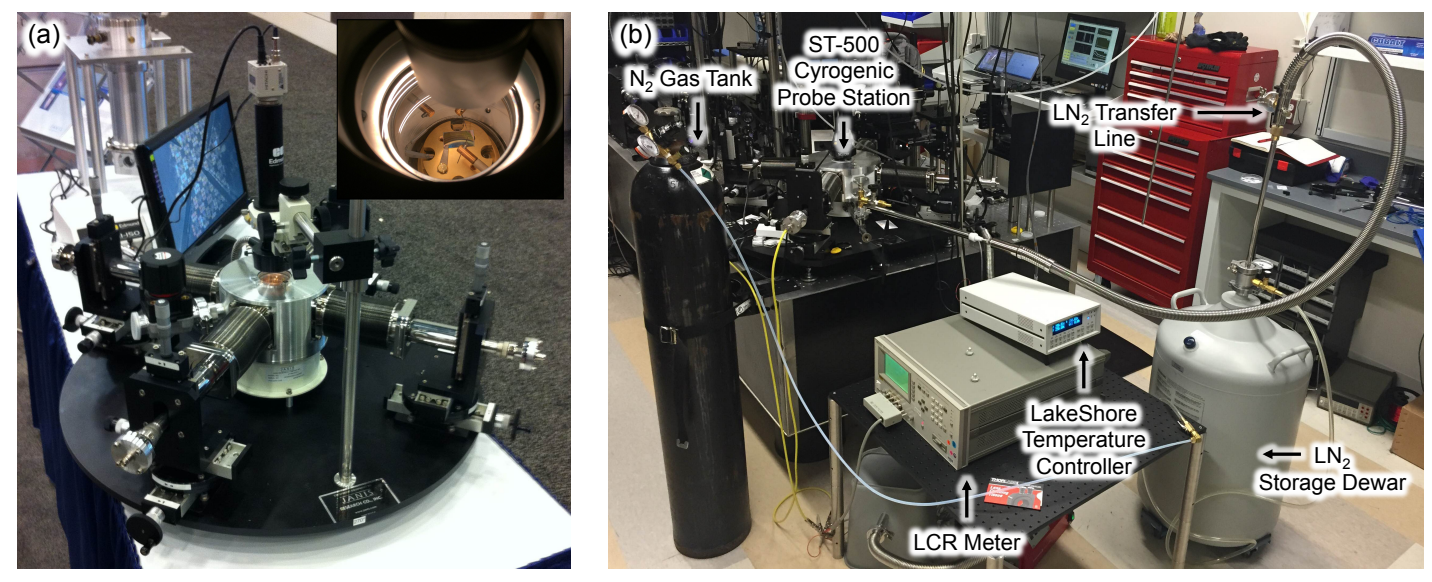

Figure 3.6: (a) Photograph of the ST-500 Cryogenic Probe System manufactured by Janis Research Company, LLC. Inset: View of the sample chuck and probe tips within the ST-500 through the top-side optical window. (b) Photograph of ST-500 integrated into the TDTR system, complete with all peripheral equipment required to perform temperature control and electrical bias for the measurements.

The thermal measurements of the bilayer PZT heterostructures made over a range of temperatures while under applied electrical bias were made using the ST-500 Cryogenic Probe System manufactured by Janis Research Company, LLC. This piece of equipment provides accurate, repeatable electrical probing of the PZT materials down to cryogenic temperatures while under vacuum. The unit contains four independently-manuverable probe arms with triaxial connectors that are appropriate for signals from DC - $20 \mathrm{MHz}$. The sample is mounted within the chamber onto a 2" dia. gold-plated chuck which can be independently biased via a triaxial connection. Once the chamber is evacuated, the tem- 
perature is controlled via a LakeShore temperature controller, striking a balance between the cooling provided by the flow of liquid nitrogen $\left(\mathrm{LN}_{2}\right)$ and heating via a resistive-heater mounted in the chuck. The $\mathrm{LN}_{2}$ in this flow-type system is pumped from a storage dewar through the cryostat by backfilling the dewar with nitrogen gas $\left(\mathrm{N}_{2}\right)$ and the flow rate is controlled via both the flow of $\mathrm{N}_{2}$ into the dewar, as well as a needle value on the transfer line between the dewar and cryostat. Additionally, a window in the top of the cryogenic chamber provides optical access to the sample, allowing us to conduct thermal measurements via TDTR while under applied electrical bias using a similar probe-down topology.
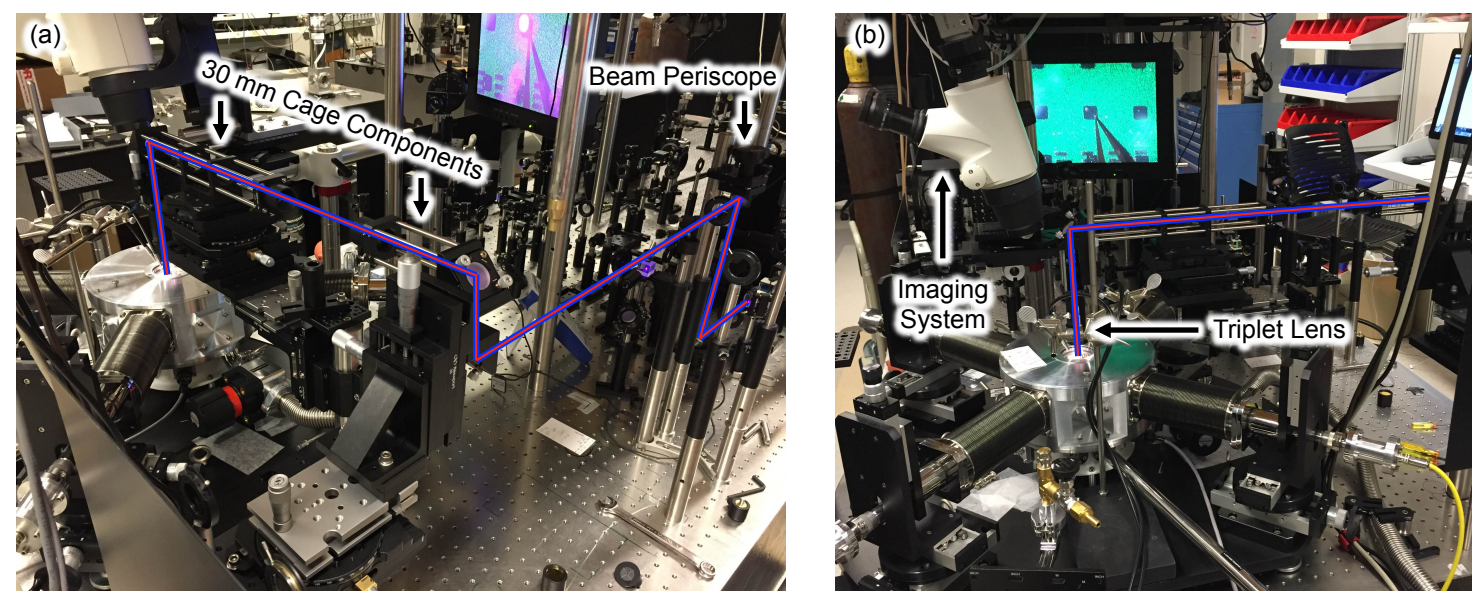

Figure 3.7: (a) Photograph of the ST-500 within the TDTR system, highlighting the optical components required to route the beam over to and down into the probe station. (b) Additional photograph showing the imaging system used to ensure accurate placement of the probe tips on the samples mounted in the cryogenic probe station, as well as the triplet lens required due to the large internal distance between the sample surface and the top optical window.

Due to the size of the cryogenic probe station (table footprint of approximately 3 feet in diameter), additional $30 \mathrm{~mm}$ cage system components were required to route the laser above the cryostat window for the probe-down measurements. The $30 \mathrm{~mm}$ cage components allow for the construction of one continuous structure, ultimately simplifying the alignment of the entire apparatus by minimizing the number of additional degrees of freedom that are introduced. Figure. 3.7 shows the position of these additional components required to route the laser above the cryogenic probe station. One challenge associated 
with the cryogenic probe station was the large distance internally between the sample surface and the optical window (approximately $75 \mathrm{~mm}$ ). As a result, the $11 \mathrm{~mm}$ focal length of the 10x Nikon objective used with the room temperature probe station was too short and could not work with the cryogenic probe station. The 10x Nikon objective was therefore replaced with a $90 \mathrm{~mm}$ effective focal length (EFL) UV-to-NIR corrected triplet lens from Edmund Optics. This lens was able to focus the laser onto the sample surface over such a large gap in space, providing comparable laser spot sizes to those using the 10x Nikon objective. As with the room temperature probe station, an off-vertical image acquisition system was integrated around the probe-down optical apparatus to provide images of the sample surface and enable accurate placement of the electrical probe tips within the cryogenic probe station.

\subsubsection{Instrumentation for Electrical Control/Measurement}

\section{HP 4284A and Keysight E4980A Precision LCR Meter}

To apply the DC electric fields to the samples during the thermal measurements, as well as monitor the properties of the material or device during the measurement, both the HP 4284A and Keysight E4980A LCR meters were used. An LCR meter is an instrument that can be used to characterize the impedance of a given device under test (DUT) and quantify the inductance $(\mathrm{L})$, capacitance $(\mathrm{C})$ and resistance $(\mathrm{R})$ of the DUT assuming a particular device model. For example, for these experiments with PZT devices, we specify a model comprised of a capacitor in parallel with a resistor that models the loss factor of the capacitive test structure. The unit makes the measurement by monitoring the magnitude and phase of the voltage across the terminals of the DUT, as well as the magnitude and phase of the current through the device. Using these phasor representations for the voltage and current across and through the device, respectively, the complex impedance of the device can be determined via Ohm's law. Once the resistive and reactive-components of the complex impedance are known, the appropriate loss term and capacitance (or inductance) 
of the DUT can be calculated, respectively.

These meters allow for the characterization of the impedance characteristics of a DUT as a function of drive frequency $(20 \mathrm{~Hz}-1 \mathrm{MHz}$ with the 4824A; $20 \mathrm{~Hz}-2 \mathrm{MHz}$ with the E4980A), AC signal amplitude (0 - $\left.20 \mathrm{~V}_{\mathrm{RMS}}\right)$ and DC bias ( $\left.\pm 40 \mathrm{~V}\right)$. In our thermal experiments, the $\mathrm{AC}$ frequency and signal amplitude are kept fixed at $1 \mathrm{kHz}$ and $100 \mathrm{mV}_{\mathrm{RMS}}$, respectively, while the DC bias level is varied and TDTR scans are taken at each applied field strength. The advantage of using an LCR meter instead of a DC voltage source to apply these electric fields is that the LCR meter provides an indication of both contact quality to the DUT with the electrical probe tips, and also allows us to monitor the health of the capacitive structure and identify the onset of dielectric breakdown through the value of the loss factor.

\section{Radiant Technologies, Inc. Precision LC II Ferroelectric Tester}

To characterize the ferroelectric properties of the thin film materials and devices, a Precision LC II Ferroelectric Tester system by Radiant Technologies was used. While the LC II can perform a wide variety of measurements on a given DUT, we were primarily interested in using the unit to measure the ferroelectric polarization $(\mathrm{P})$ as a function of electric field (E). These P-E properties are hysteretic in nature and the shape of the resulting loops can provide a great deal of information about the electric polarizability, and thus the domain behavior within a ferroelectric material or device. Figure 3.8 plots a representative ferroelectric hysteresis loop, identifying particular parameters of interest such as the coercive field strength $\left(\mathrm{E}_{\mathrm{c}}, \mathrm{kV} / \mathrm{cm}\right)$, saturation polarization $\left(\mathrm{P}_{\mathrm{sat}}, \mu \mathrm{C} \mathrm{cm}{ }^{-2}\right)$ and remanent polarization $\left(\mathrm{P}_{\mathrm{r}}, \mu \mathrm{C} \mathrm{cm} \mathrm{cm}^{-2}\right)$. The coercive field strength represents the ability of a material to withstand an applied electric field magnitude before becoming depolarized, denoted by the field strength were the polarization $(\mathrm{P})$ equals zero. The saturation polarization represents the point at which for increasing electric field magnitudes, the polarization remains unchanged and is at its maximum. The remanent polarization is the remaining polarization 
within the material that is still present after the applied electric fields have been removed, denoted by the intersection of the hysteresis loop with the vertical axis when the electric field (E) equals zero.

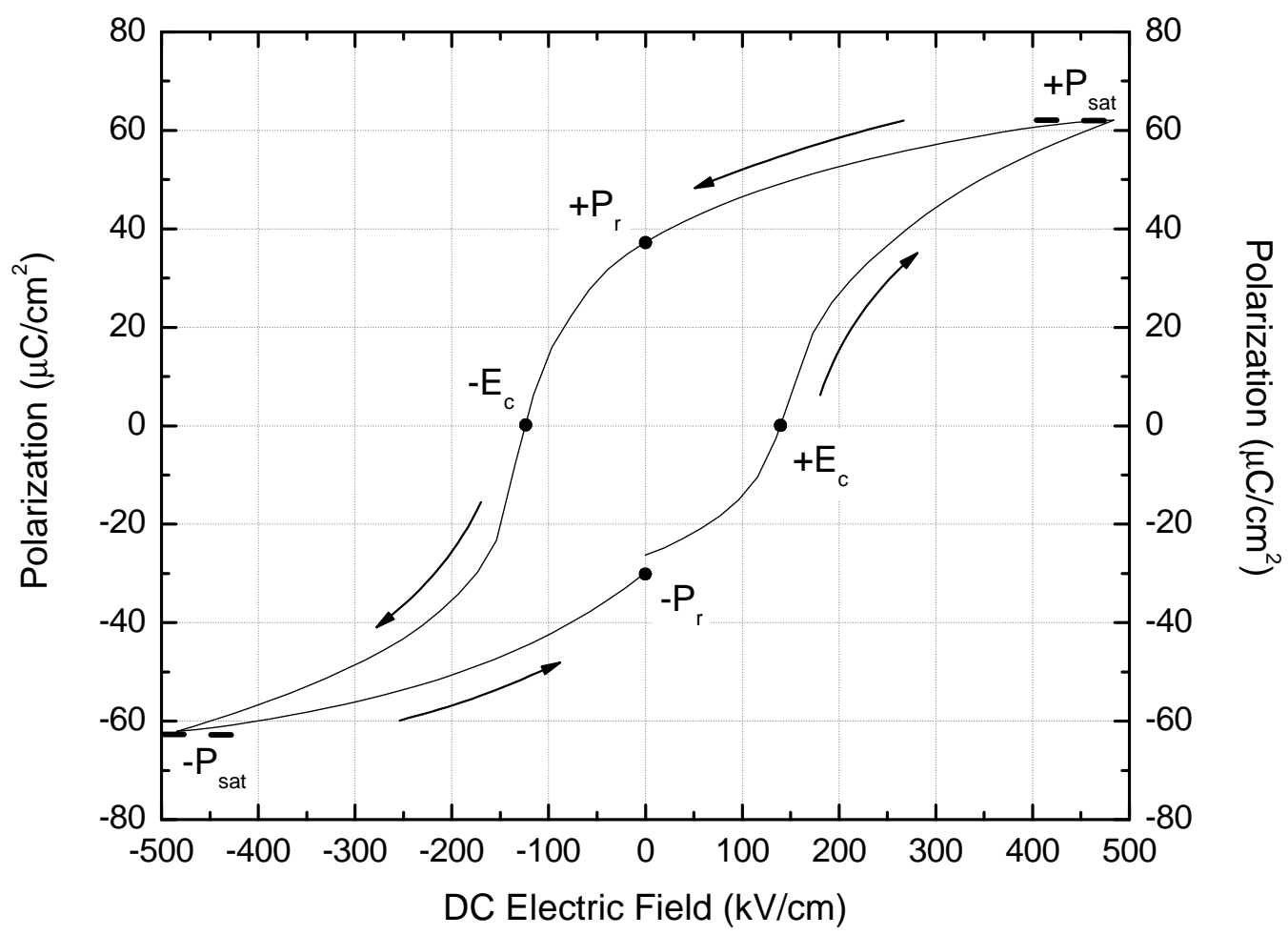

Figure 3.8: Example of Polarization vs. Electric Field (P-E) loop data collected from a PZT device to illustrate important features of the hysteresis curve, including the coercive field $\left( \pm \mathrm{E}_{\mathrm{c}}\right)$, saturation polarization $\left( \pm \mathrm{P}_{\text {sat }}\right)$ and remanent polarization $\left( \pm \mathrm{P}_{\mathrm{r}}\right)$. Arrows indicate the progression of the applied electric field, sweeping from zero field to maximum positive field, then maximum negative field, and then back to zero field.

In this work, the P-E loops were primarily used to confirm the saturation of the polarization, and thus the switching of the domains in the PZT bilayers measured over a range of temperatures. While the LCR meter was used during the thermal measurements, the ferroelectric hysteresis measurements were made separate from the thermal measurements, using the same cryogenic probe station. 


\section{Chapter 4}

\section{Thermal Transport in Lead Zirconate Titanate $\left(\mathbf{P b Z r}_{1-x} \mathbf{T i}_{x} \mathbf{O}_{3}\right)$}

\subsection{Motivation \& Background}

Lead zirconate titanate $\left(\mathrm{PbZr}_{1-x} \mathrm{Ti}_{x} \mathrm{O}_{3}, \mathrm{PZT}\right)$ is an important and versatile solid-solution material system. Since its discovery in the early 1950 s as an alternative to barium titanate for ceramic filters [70], PZT has been and continues to be a critical component in the development of many different technologies, including capacitors [41], electro-optic shutters [71], ultrasonic transducers [72], micro electro-mechanical systems (MEMS) [73], and ferroelectric random access memory (FeRAM) [74]. While the past decade has seen an increasing amount of research into lead-free piezoceramic alternatives, the reality is that PZT remains the material of choice within the $\$ 20$ billion piezoactuator market due to its stability, scalability and cost [75].

PZT is a binary solid-solution system comprised of a particular molar fraction $(x)$ of lead titanate $\left(\mathrm{PbTiO}_{3}, \mathrm{PTO}\right)$ relative to the fraction of lead zirconate $\left(\mathrm{PbZrO}_{3}, \mathrm{PZO}\right)$. The material has an $\mathrm{ABO}_{3}$ type perovskite structure with lead at the $A$-site (corner of the cube), zirconium or titanium occupying the $B$-site (body center) and oxygen at the face centers. Figure 4.1 depicts the phase diagram for the PZT material system as a function of $x$ and 


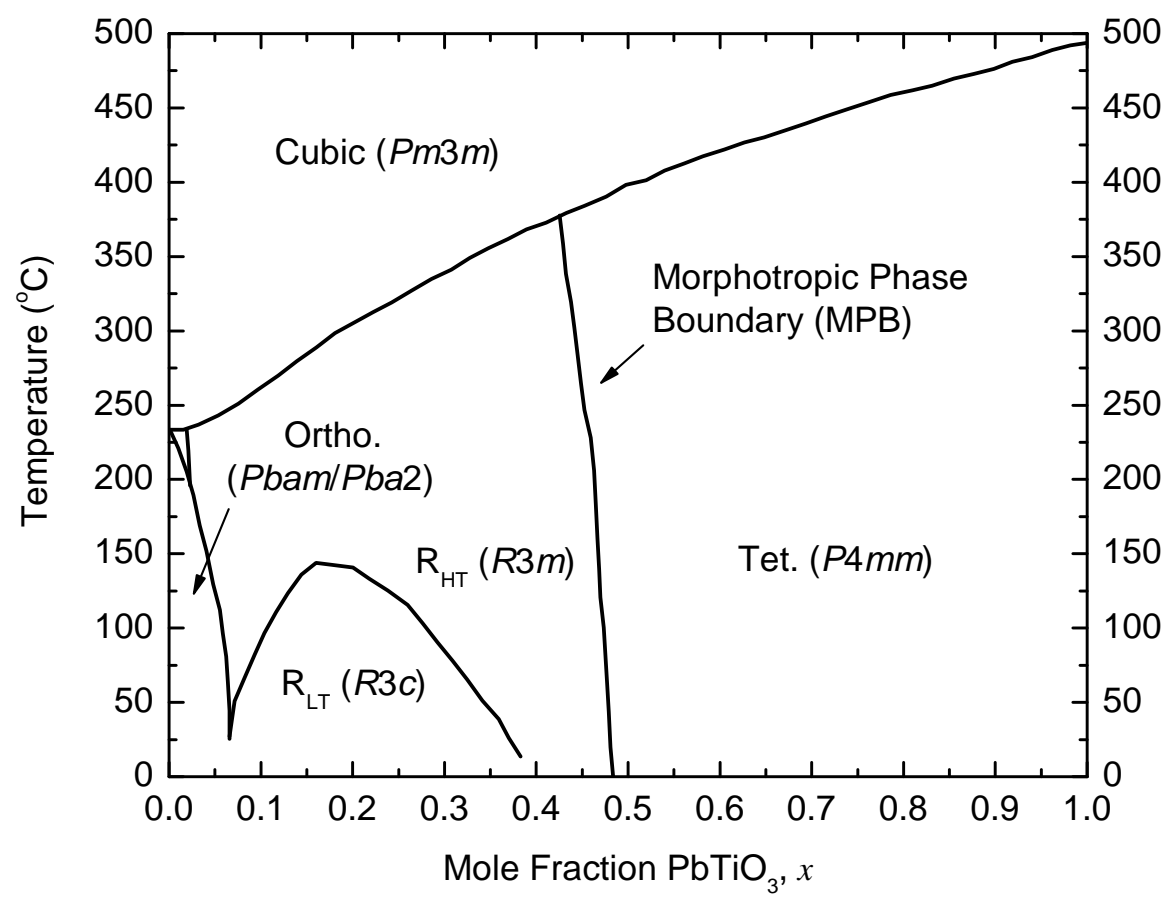

Figure 4.1: Phase diagram of lead zirconate titanate $\left(\mathrm{PbZr}_{1-x} \mathrm{Ti}_{x} \mathrm{O}_{3}, \mathrm{PZT}\right)$, adapted from [21].

temperature, illustrating the variety of crystal structures that are possible [21]. In the topmost region of the diagram above the line denoting the Curie temperature as a function of composition, PZT has a cubic structure $(\mathrm{Pm} 3 \mathrm{~m})$ and is paraelectric with no spontaneous electric polarization. However, as PZT is cooled below its Curie temperature, there is a distortion of the crystal into one of several structures depending on the composition. For titanium-rich compositions $(x>0.48)$, the crystal has a tetragonal structure $(P 4 m m)$, whereas for zirconium-rich compositions, the crystal can exhibit either orthorhombic $(x<$ $0.05, \mathrm{Pbam} / \mathrm{Pba} 2)$ or rhombohedral symmetry $(0.05<x<0.48, R 3 c$ or $R 3 m$ for low and high-temperature phases, respectively) [76]. The vertical line in Figure 4.1 in the vicinity of $x=0.48$ at $20^{\circ} \mathrm{C}$ denotes the morphotropic phase boundary (MPB) where the material exhibits an enhancement in the dielectric constant $\left(\varepsilon_{r}\right)$ and piezoelectric coefficient $\left(d_{33}\right)$ compared to compositions in the adjacent tetragonal and rhombohedral regions. The variety 
of crystal structures possible and commensurate tunability of several important properties make the PZT system an integral part of a broad array of technologies.

While the mixture of $\mathrm{PbZrO}_{3}$ and $\mathrm{PbTiO}_{3}$ may result in many functional properties, it is important to consider the impact on the thermal properties as well. The reduction of $\kappa$ via mass-disorder in binary semiconductor alloys such as $\mathrm{Si}_{1-x} \mathrm{Ge}_{x}\left([\overline{77]})\right.$ and $\mathrm{Al}_{x} \mathrm{Ga}_{1-x} \mathrm{~N}$ ([78]) has been shown experimentally to result in an order of magnitude reduction in $\kappa$ for compositions of roughly $0.20<x<0.80$ due to the strong scattering of the high frequency phonons present in the material. Furthermore, several works have also investigated the interplay of alloy scattering and boundary scattering in nanostructured forms of the aforementioned materials, including thin films [78, 79] and nanowires [80]. Through their experimental results and contextualization using a Debye-based Callaway model, these works demonstrate that $\kappa$ can be further reduced via boundary scattering by limiting the mean free path of low-frequency phonons that are not otherwise affected by the alloy atoms. Since PZT is often employed in thin film, polycrystalline form for use in a wide array of applications, it is important to also consider these contributions to the phonon scattering landscape in addition to scattering via mass-disorder in the solid-solution system.

Knowledge of the thermal conductivity dependence on composition and microstructure is limited for PZT [25, 26, 81, 82]. Thermal conductivity can have a significant effect on the performance of a device, both in the short term as it pertains to things like sensitivity, loss tangent and operational stability, but also in the long term concerning the reliability and degradation of the device over time. In this chapter, we measure the thermal conductivity of randomly-oriented polycrystalline PZT thin films with compositions ranging over the entire phase diagram $(0 \leq x \leq 1)$. The PZT thin films were deposited on platinized silicon substrates through chemical solution deposition (CSD), which is a commonly used means to prepare device quality PZT films [41, 83]. Using time-domain thermoreflectance (TDTR), we measure the thermal conductivities $(\kappa)$ of PZT thin films of varying solidsolution composition. We show that the thermal conductivity of the PZT system exhibits an alloy/mixture type trend where $\kappa$ for compositions $0.20 \leq x \leq 0.80$ is $37.5 \%$ lower than 


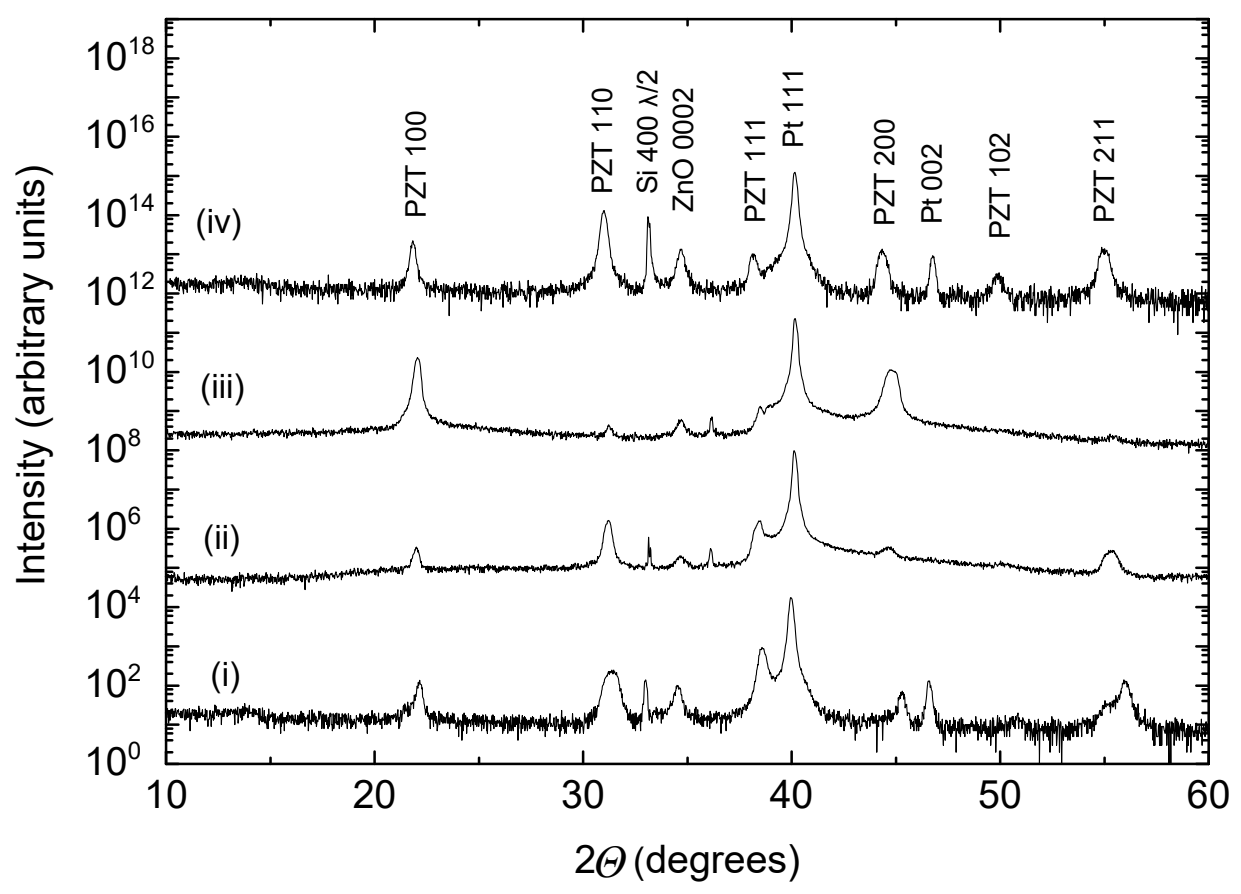

Figure 4.2: Representative $2 \theta-\theta$ XRD analysis of PZT thin films with compositions (i) $\mathrm{PbZr}_{0.30} \mathrm{Ti}_{0.70} \mathrm{O}_{3}, \mathrm{PbZr}_{0.52} \mathrm{Ti}_{0.48} \mathrm{O}_{3}$ at pyrolysis temperatures of (ii) $350^{\circ} \mathrm{C}$ and (iii) $400^{\circ} \mathrm{C}$ and (iv) $\mathrm{PbZr}_{0.70} \mathrm{Ti}_{0.30} \mathrm{O}_{3}$. Compositions near the MPB with a pyrolysis temperature of $350^{\circ} \mathrm{C}$ were characterized as randomly oriented, while compositions with a pyrolysis temperature of $400^{\circ} \mathrm{C}$ possessed a (100)-texture.

that measured for $\mathrm{PbTiO}_{3}(x=1)$ thin films of comparable thickness and grain size. We examine the trend in the data through a Debye-based alloy/mixture model for $\kappa$ to gather insight regarding the relative strength of several phonon scattering mechanisms for various compositions of PZT.

\subsection{Sample Fabrication \& Characterization}

PZT thin films with a target thickness of $300 \mathrm{~nm}$ were deposited on $\mathrm{Pt} / \mathrm{ZnO} / \mathrm{SiO}_{2} / \mathrm{Si}$ substrates using an inverse mixing order (IMO) chemical solution. PZT film compositions ranging from $\mathrm{PbZr}_{1-x} \mathrm{Ti}_{x} \mathrm{O}_{3}$ for $0 \leq x \leq 1$ with 10 mole percent steps (save for a single film 


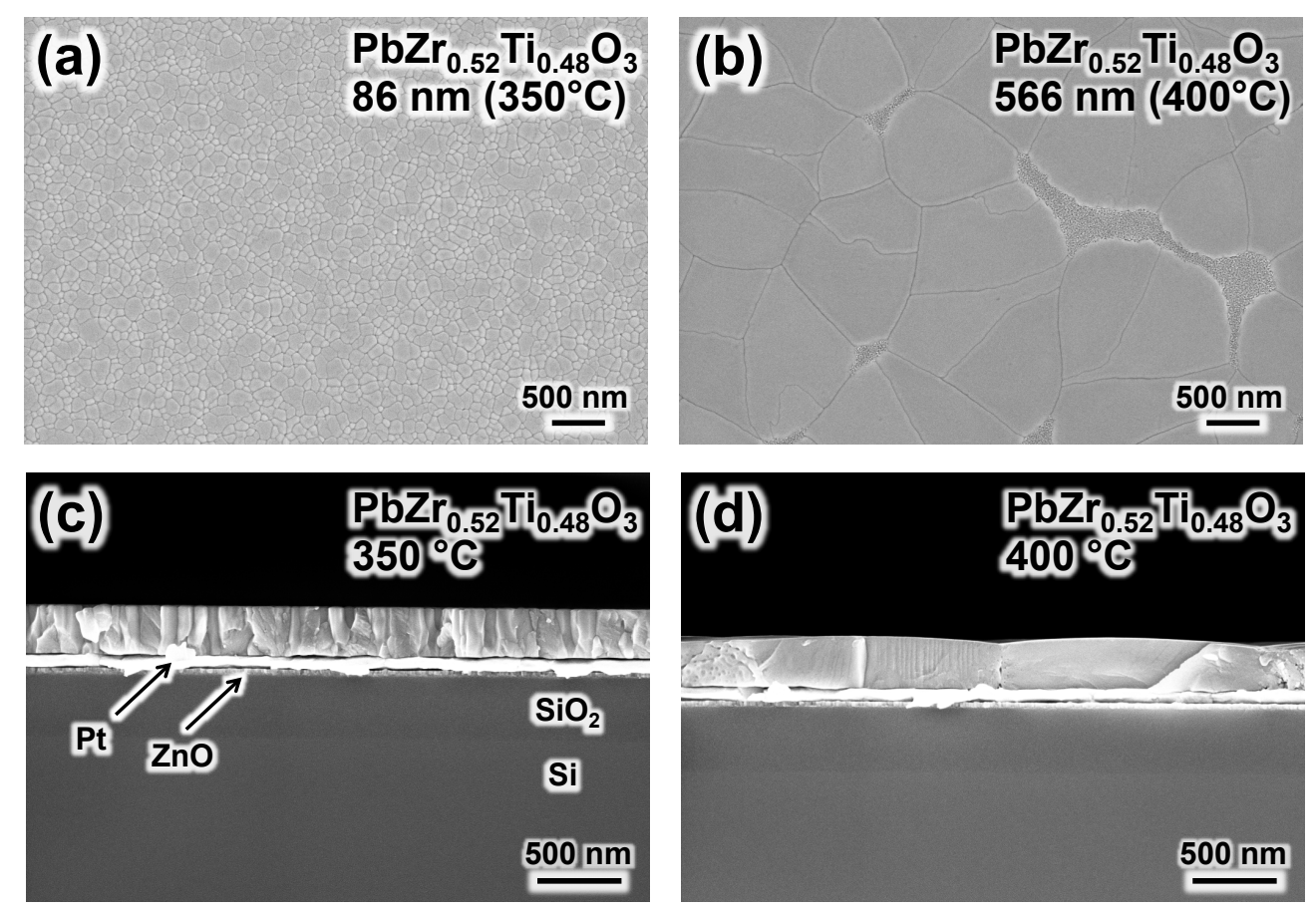

Figure 4.3: Plan-view (a,b) and cross-sectional (c,d) scanning electron microscopy (SEM) images of the MPB-composition thin films at the two pyrolysis temperatures, highlighting the significant difference in average grain size.

with $x=0.05$ ) were prepared with a pyrolysis temperature of $350^{\circ} \mathrm{C}$. Smaller compositional steps, 4 mole percent, were also investigated near the morphotropic phase boundary (MPB, $x=0.48)$ composition and two pyrolysis temperatures were investigated: $350^{\circ} \mathrm{C}$ and $400^{\circ} \mathrm{C}$ for 1 minute, which has been shown previously to result in significantly different grain morphologies. [83] Details of PZT film preparation can be found in [83]. Metal-insulator-metal stacks were fabricated with both $300 \times 300 \mu \mathrm{m}$ platinum and aluminum top electrodes deposited via RF magnetron sputtering and e-beam evaporation, respectively, for electrical and thermal characterization. Figure 4.2 shows representative $2 \theta-\theta$ XRD analysis for $\mathrm{PbZr}_{0.30} \mathrm{Ti}_{0.70} \mathrm{O}_{3}$ and $\mathrm{PbZr}_{0.70} \mathrm{Ti}_{0.30} \mathrm{O}_{3}$ compositions, and MPB composition for a pyrolysis temperature of $350^{\circ} \mathrm{C}$ as well as the MPB composition with a pyrolysis temperature of $400^{\circ} \mathrm{C}$.

Compositions near the MPB with a pyrolysis temperature of $350^{\circ} \mathrm{C}$ were characterized as randomly oriented, while compositions with a pyrolysis temperature of $400^{\circ} \mathrm{C}$ possessed 
a (100)-texture. Film thicknesses and grain sizes were measured using secondary electron scanning electron microscopy (SEM) and atomic force microscopy. Figure 4.3 (a,b) and (c,d) shows representative SEM images plan view and cross-sectional images, respectively, for $\mathrm{PbZr}_{0.52} \mathrm{Ti}_{0.48} \mathrm{O}_{3}$ made with a pyrolysis temperature of $350^{\circ} \mathrm{C}$ and $400^{\circ} \mathrm{C}$. Grain sizes were determined using a linear intercept method. [84] For the $350^{\circ} \mathrm{C}$ pyrolysis temperature, grain sizes ranged from $47 \pm 5 \mathrm{~nm}$ to $98 \pm 12 \mathrm{~nm}$ for $0 \leq x \leq 1$, where the largest grain size occurred near the MPB composition. Grain sizes for a pyrolysis temperature of $400^{\circ} \mathrm{C}$ ranged from $530 \pm 80 \mathrm{~nm}$ to $2200 \pm 700 \mathrm{~nm}$ for $0.46 \leq x \leq 0.58$. Grain sizes for representative compositions are listed in Figure $4.3(\mathrm{a}-\mathrm{d})$ and Table 4.1 .

Table 4.1: Pyrolysis temperature $\left(T_{\mathrm{P}}\right)$, film thickness $\left(d_{f}\right)$ and average grain sizes $\left(d_{g}\right)$, along with the associated measurement uncertainties $\left(\sigma_{d}, \sigma_{g}\right)$.

\begin{tabular}{cccccc}
$x$ & $T_{\mathrm{P}}\left({ }^{\circ} \mathrm{C}\right)$ & $d_{f}(\mathrm{~nm})$ & $\sigma_{d}(\mathrm{~nm})$ & $d_{g}(\mathrm{~nm})$ & $\sigma_{g}(\mathrm{~nm})$ \\
\hline 0.00 & 350 & 328 & 16 & 2470 & 335 \\
0.05 & 350 & 278 & 42 & - & - \\
0.10 & 350 & 335 & 11 & $2680 / 225$ & $760 / 37$ \\
0.20 & 350 & 344 & - & 229 & 23 \\
0.30 & 350 & 346 & - & 88 & 7 \\
0.40 & 350 & 318 & - & 84 & 6 \\
0.42 & 350 & - & - & - & - \\
- & 400 & 298 & 18 & 202 & 32 \\
0.45 & 350 & 296 & 8 & 98 & 11 \\
- & 400 & 310 & 21 & 824 & 82 \\
0.48 & 350 & 294 & 4 & 86 & 5 \\
- & 400 & 316 & 5 & 566 & 45 \\
0.51 & 350 & 289 & 2 & 83 & 4 \\
- & 400 & 283 & 18 & 328 & 30 \\
0.54 & 350 & 316 & 13 & 85 & 5 \\
- & 400 & 307 & 13 & 294 & 30 \\
0.60 & 350 & 307 & - & 99 & 10 \\
0.70 & 350 & 325 & - & 97 & 5 \\
0.80 & 350 & 293 & - & 144 & 26 \\
0.90 & 350 & 348 & 50 & 152 & 24 \\
1.00 & 350 & 254 & 51 & 119 & 31
\end{tabular}




\subsection{Thermal Measurements \& Analysis}

The thermal conductivities of the samples were determined using time domain thermoreflectance (TDTR). To facilitate the measurement, the samples were coated with approximately $80 \mathrm{~nm}$ of aluminum to serve as the transducer for the thermoreflectance measurement. In order to fit the model to the data for the thermal parameters of interest, the other thermal properties of the materials in the test structure must be well known. The thicknesses of the top aluminum layer was determined via pico-second acoustics [85, 86] over the first 20-30 ps of pump-probe delay in the TDTR measurement, while the thermal conductivity was inferred based on prior measurements of similar E-beam deposited films (approximately $150 \mathrm{~W} \mathrm{~m}^{-1} \mathrm{~K}^{-1}$ for $80 \mathrm{~nm}$ of aluminum). We assume literature values for the heat capacities of Al [87], as well as the heat capacity of PZT of different compositions $(x)$ which was calculated as a ratiometric mixture of the room temperature heat capacities of $\mathrm{PbZrO}_{3}$ and $\mathrm{PbTiO}_{3}$. [88]

For this study, we amplitude-modulate the pump beam with an $8 \mathrm{MHz}$ sinusoid and monitor the ratio of the in-phase to out-of-phase signal of the probe beam with a lock-in amplifier. We fit the TDTR model to the data by varying the thermal conductivity of the PZT films and the thermal boundary conductance between the top metal film and the PZT samples. While the $1 / e$ thermal penetration depth of our pump beam at $8 \mathrm{MHz}$ is less than the PZT film thickness for all the compositions in this study (approx. $135 \mathrm{~nm}$ for 0 $<x<0.7$, increasing to approx. $180 \mathrm{~nm}$ at $x=1$ ), our analysis suggests that the TDTR data could be sensitive to thermal transport across the platinized-silicon stack (100 nm Pt/ $40 \mathrm{~nm} \mathrm{ZnO} \mathrm{/} 300 \mathrm{~nm} \mathrm{SiO}{ }_{2}$ / bulk Si) upon which the PZT films are deposited. Therefore, the platinized-silicon samples were also characterized via TDTR to eliminate the number of unknowns in the thermal model for the PZT thin films.

To perform these calibrations, platinized-silicon samples both with and without the 300 $\mathrm{nm} \mathrm{SiO} 2$ were fabricated for TDTR measurements. For these samples, a two-layer model of a platinum thin film on either $\mathrm{SiO}_{2}$ or $\mathrm{Si}$ was employed, where the thermal boundary 
conductance between the layers $\left(G_{1}\right)$ is fit to the TDTR data (heat capacity, thermal conductivity and thickness of the platinum top layer are all known, as are the heat capacity and thermal conductivity of $\mathrm{SiO}_{2}$ and $\mathrm{Si}$ ). This two-layer approach was used in order to lump the thermal conductivity of the $40 \mathrm{~nm} \mathrm{ZnO}$ layer in with the conductances across the $\mathrm{Pt} / \mathrm{ZnO}$ and $\mathrm{ZnO} / \mathrm{SiO}_{2}$ or $\mathrm{ZnO} / \mathrm{Si}$ interfaces into a single total conductance. At $8 \mathrm{MHz}$, the buried $300 \mathrm{~nm} \mathrm{SiO}$ layer can be considered "thermally-thick" and it is therefore reasonable to consider it as a semi-infinite bottom layer in the model.

In practice, the measurements made on the sample with $300 \mathrm{~nm} \mathrm{SiO}_{2}$ were unable to provide a reliable value for $G_{1}$ due to the competing sensitivity of the thermal data to the properties of the $\mathrm{SiO}_{2}$. Alternatively, fitting the data for the sample without the $\mathrm{SiO}_{2}$ provided much more accurate results due to the fact that the sensitivity to $G_{1}$ is increased because of the two order of magnitude increase in the $\kappa$ of the semi-infinite layer $(1.4$ $\mathrm{W} \mathrm{m} \mathrm{m}^{-1} \mathrm{~K}^{-1}$ for $\mathrm{SiO}_{2}, 145 \mathrm{~W} \mathrm{~m} \mathrm{~K}^{-1}$ for $\mathrm{Si}$ ). Fitting this data resulted in a lumped conductance of the $40 \mathrm{~nm} \mathrm{ZnO}$ film, along with its adjacent boundary conductances of $G_{\mathrm{ZnO}}=96 \mathrm{MW} \mathrm{m}^{-2} \mathrm{~K}^{-1}$.

For fitting the PZT thin film data, a three-layer model is used; layer 1 is the aluminum thin film, layer 2 is the PZT thin film and layer 3 is $\mathrm{SiO}_{2}$ (semi-infinite). Due to the large conductances of the platinum and $\mathrm{ZnO}$ thin films $\left(G_{\mathrm{Pt}}=\kappa_{\mathrm{Pt}} / d_{\mathrm{Pt}}=41 \mathrm{~W} \mathrm{~m}^{-1} \mathrm{~K}^{-1} / 100 \mathrm{~nm}\right.$ $=410 \mathrm{MW} \mathrm{m}^{-2} \mathrm{~K}^{-1} ; G_{\mathrm{ZnO}}=96 \mathrm{MW} \mathrm{m}^{-2} \mathrm{~K}^{-1}$ ) compared to the effective conductance of the PZT thin films $\left(G_{\mathrm{PZT}}=\kappa_{\mathrm{PZT}} / d_{\mathrm{PZT}}=1.3 \mathrm{~W} \mathrm{~m}^{-1} \mathrm{~K}^{-1} / 300 \mathrm{~nm}=4.33 \mathrm{MW} \mathrm{m}^{-2}\right.$ $\mathrm{K}^{-1}$ ), it is reasonable to combine $G_{\mathrm{Pt}}$ and $G_{\mathrm{ZnO}}$ into a single conductance for use in the three-layer model between the PZT and $\mathrm{SiO}_{2}$ layers $\left(G_{2}=77.8 \mathrm{MW} \mathrm{m}^{-2} \mathrm{~K}^{-1}\right)$ via:

$$
G_{2}=\left(G_{\mathrm{Pt}}+G_{\mathrm{ZnO}}\right)^{-1}
$$

With $G_{2}$ throughly characterized, the thermal conductivity of the PZT thin films can be extracted from the TDTR data with the three-layer model to a high degree of confidence. 


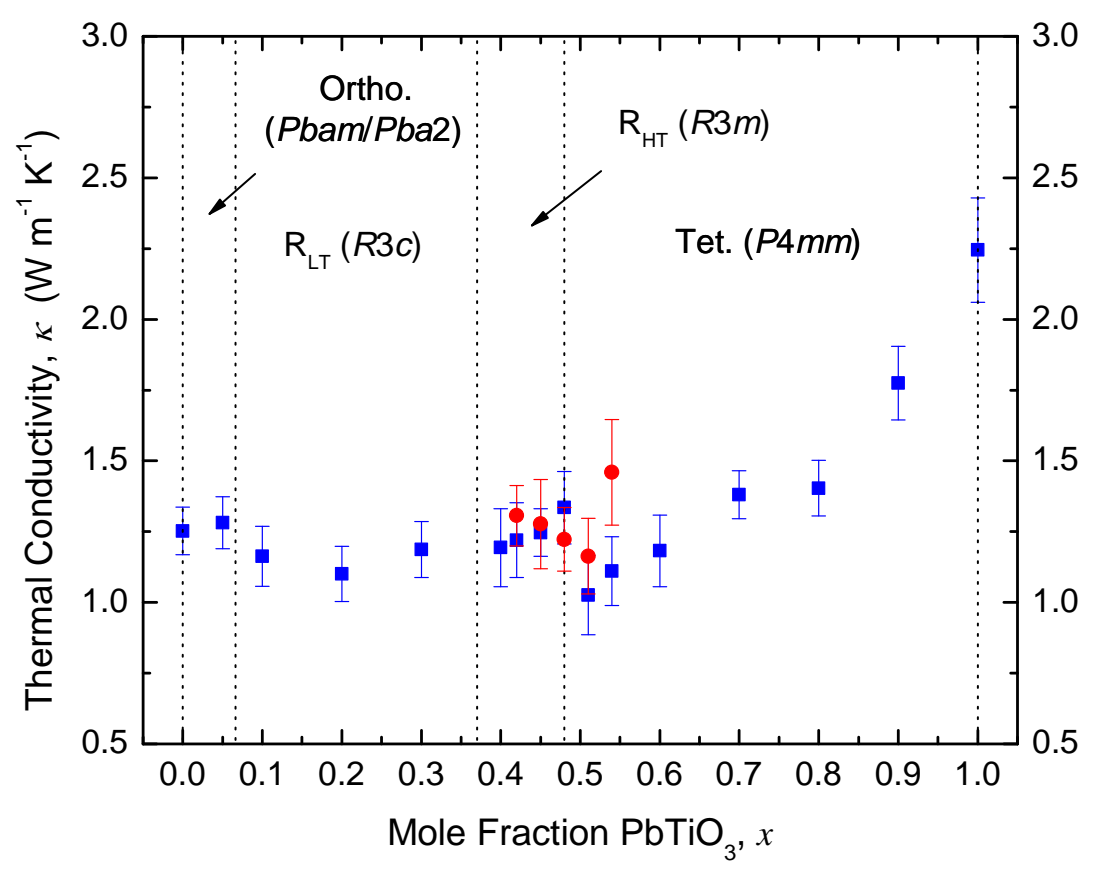

Figure 4.4: Thermal conductivity $(\kappa)$ of PZT thin films with various solid-solution compositions, $x$, fabricated with pyrolysis temperatures of $350^{\circ} \mathrm{C}$ (blue squares) and $400^{\circ} \mathrm{C}$ (red circles). The dashed vertical lines denote the locations of the phase boundaries from Fig. 4.1 at $20^{\circ} \mathrm{C}$.

\subsection{Results \& Discussion}

Figure 4.4 shows the measured thermal conductivities of the PZT thin films as a function of their solid solution composition fabricated with pyrolysis temperatures of $350^{\circ} \mathrm{C}$ (blue squares) and $400^{\circ} \mathrm{C}$ (red circles). Starting with the $\mathrm{PbTiO}_{3}$ thin film $(x=1.00)$, we measure a thermal conductivity of $2.24 \mathrm{~W} \mathrm{~m}^{-1} \mathrm{~K}^{-1}$. This value is $55.7 \%$ lower than the bulk value of $5.06 \mathrm{~W} \mathrm{~m}^{-1} \mathrm{~K}^{-1}$ reported in [22] which we attribute to phonon scattering at the film and grain boundaries in our samples. As the molar fraction of $\mathrm{PbZrO}_{3}$ increases ( $x$ decreases), we observe a decrease in the thermal conductivity compared to $\mathrm{PbTiO}_{3}(x$ $=1.00$ ). Over the range $0.80<x<1.00$, the thermal conductivity drops from $2.24 \mathrm{~W}$ $\mathrm{m}^{-1} \mathrm{~K}^{-1}$ to $1.40 \mathrm{~W} \mathrm{~m}^{-1} \mathrm{~K}^{-1}$; a reduction of $37.5 \%$. Such a steep reduction in the thermal conductivity of an initially stoichiometrically pure material system with the addition 
of an additional species has been shown to occur in several systems [77, 78] and is often explained in the context of alloy/impurity phonon scattering. In this case, the fraction of $B$-sites in the $A B \mathrm{O}_{3}$ perovskite system populated with $\mathrm{Zr}$ instead of Ti atoms increases as $x$ decreases and these $\mathrm{Zr}$ atoms serve as mass-impurity scattering sites for phonons. We do not believe that this reduction between the $x=1.0$ and $x=0.80$ samples is due to an increase in phonon scattering caused by physical boundaries because of the fact that the $x$ $=0.80$ sample is thicker and has larger grains than the $x=1.0$ sample.

Across the range for $x<0.80$, the trend in thermal conductivity remains largely flat (approximately $1.2 \pm 0.2 \mathrm{~W} \mathrm{~m}^{-1} \mathrm{~K}^{-1}$ ) with the exception of an apparent discontinuity in the vicinity of the MPB $(0.45<x<0.54)$. Interestingly, the thermal conductivity does not increase as $x$ decreases over the range $0<x<0.20$, approaching pure $\mathrm{PbZrO}_{3}$ at $x=0$. In most alloy systems [77, 78], the trend in thermal conductivity as a function of composition will create a U-shape where the thermal conductivities of the constitutive components $(x=$ $0,1)$ are both much greater than the thermal conductivity over $0.20<x<0.80$. However, the PZT system does not exhibit this U-shaped symmetry as a function of composition with the thermal conductivity of thin film $\mathrm{PbZrO}_{3}$ measuring $44 \%$ lower than $\mathrm{PbTiO}_{3}(1.25$ and $2.25 \mathrm{~W} \mathrm{~m}^{-1} \mathrm{~K}^{-1}$, respectively).

While there are published measurements of the thermal conductivity of bulk $\mathrm{PbZrO}_{3}$ from 2 - $40 \mathrm{~K}([23])$ and $345-530 \mathrm{~K}([24])$, there remains a gap in measured thermal conductivities from 40 - $345 \mathrm{~K}$. Nonetheless, the overall trend with temperature considering these two published data sets suggests a high degree of phonon scattering within the material, leading to an amorphous/glass-like trend in thermal conductivity. Figure 4.5 plots the aforementioned bulk thermal conductivity data for $\mathrm{PbZrO}_{3}$ [23, 24] and $\mathrm{PbTiO}_{3}$ [22]. This glass-like trend is markedly different from that of bulk $\mathrm{PbTiO}_{3}$ which exhibits a clear maximum in thermal conductivity around $40 \mathrm{~K}$ and decreases with increasing temperature. As a result, it is reasonable to postulate that the considerable difference in the thermal conductivities of thin film $\mathrm{PbZrO}_{3}$ and $\mathrm{PbTiO}_{3}$ is driven by the intrinsic properties of the materials and not an extrinsic size effect. 


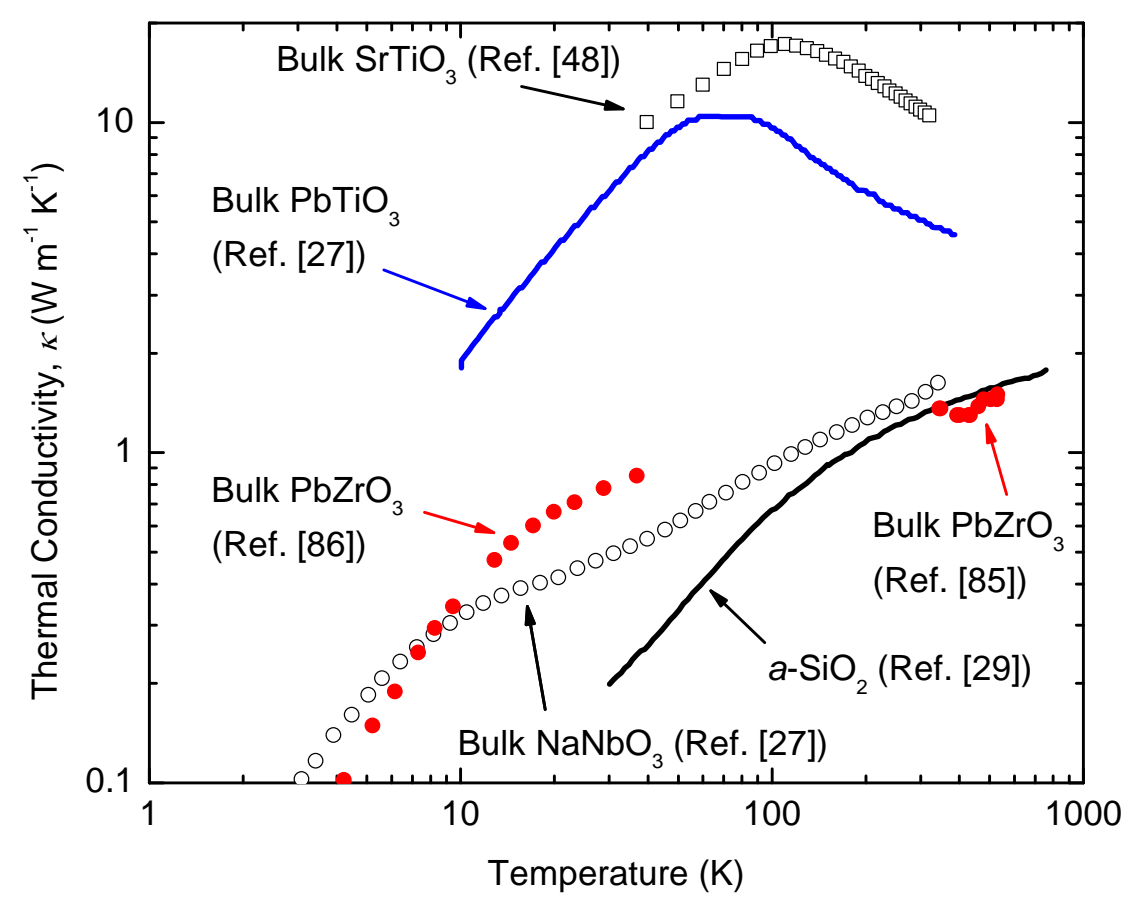

Figure 4.5: Bulk thermal conductivity data over temperature for $\mathrm{PbTiO}_{3}$ ([22], blue line) and $\mathrm{PbZrO}_{3}$ ([23, 24], red circles) highlighting the large difference in the temperature trends between stoichiometrically-similar materials $\left(\mathrm{ABO}_{3}\right.$ perovskite oxides). Also plotted for comparison are $\mathrm{SrTiO}_{3}$ ([15], open squares), $\mathrm{NaNbO}_{3}$ ([22], open circles) and $a-\mathrm{SiO}_{2}$ ([6], black line).

To confirm this, bulk samples of $\mathrm{PbZrO}_{3}(x=0), 70 / 30 \mathrm{PZT}(x=0.30)$ and 30/70 PZT $(x=0.70)$ were fabricated to confirm that the observed trends in the thin film samples extended to the bulk as well. The thermal conductivities of these samples are plotted in Fig. 4.6 (black diamond), along with the bulk data for $\mathrm{PbTiO}_{3}$ from [22] (white diamond) and bulk data for samples around ([25], upward triangles) and at ([26], downward triangle) the MPB. Similar to the thin film data, the thermal conductivity decreases dramatically for $x<1.0$, ultimately asymptoting to a thermal conductivity of $1.57 \pm 0.13 \mathrm{~W} \mathrm{~m}^{-1} \mathrm{~K}^{-1}$ for bulk $\mathrm{PbZrO}_{3}$. This value for $\mathrm{PbZrO}_{3}$ compares well with the porosity-corrected value reported in [24] of $1.79 \mathrm{~W} \mathrm{~m}^{-1} \mathrm{~K}^{-1}$ measured at a temperature of $345 \mathrm{~K}$.

The large difference in bulk thermal conductivities between stoichiometrically-similar 


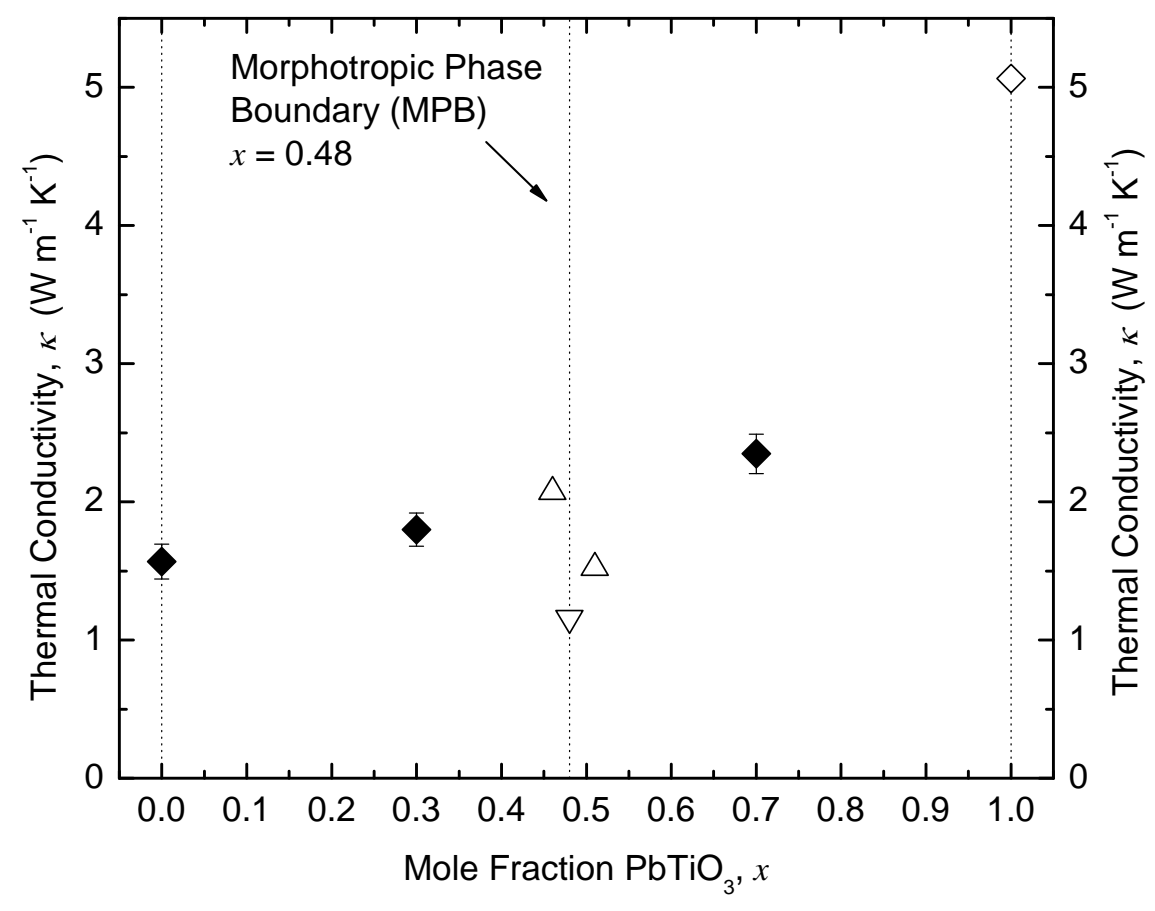

Figure 4.6: Thermal conductivity of bulk PZT of various compositions. Samples measured in this work (black diamonds) include $\mathrm{PbZrO}_{3}(x=0)$, 70/30 PZT $(x=0.30)$ and 30/70 PZT $(x=0.70)$. Values measured in the literature include $\mathrm{PbTiO}_{3}$ [22] $(x=1$, white diamond) and bulk data for samples around ([25], upward triangles) and at ([26], downward triangle) the MPB.

materials such as $\mathrm{PbTiO}_{3}$ and $\mathrm{PbZrO}_{3}$ can begin to be explained by the nature of their respective ferro-distortions that make them technologically relevant. In the case of $\mathrm{PbTiO}_{3}$, as the crystal goes from cubic to tetragonal below the Curie temperature (approx. $500^{\circ} \mathrm{C}$ ), there is a shift of the $\mathrm{TiO}_{6}$ octahedra along the $c$-axis which ultimately results in a spontaneous polarization vector along the same crystal axis. Though the crystal is distorted, the number of atoms in the unit cell (5) remains unchanged, translational symmetry is maintained and the unit cell volume remains approximately the same (cubic: $0.0625 \mathrm{~nm}^{3}$; tetragonal: $0.0633 \mathrm{~nm}^{3}$; calculated using lattice parameters from [89]). Even with this distortion, the trend in thermal conductivity over temperature remains crystalline and is comparable to perfectly cubic perovskites with no distortion at room temperature, such as 
strontium titanate $\left(\mathrm{SrTiO}_{3},[15]\right)$.

By comparison, $\mathrm{PbZrO}_{3}$ goes from cubic to orthorhombic below its Curie temperature (approx. $200^{\circ} \mathrm{C}$ ) where the $\mathrm{ZrO}_{6}$ octahedra are tilted relative to the pseudo-cubic axes in the $a^{-} a^{-} c^{o}$ scheme of Glazer $([90,91])$. The tilting of the octahedra break the translational symmetry, resulting in an orthorhombic structure with more atoms in the unit cell (40) and a much larger unit cell volume (cubic: $0.0720 \mathrm{~nm}^{3}$; orthorhombic: $0.5651 \mathrm{~nm}^{3}$ ). The enlarged unit cell volume in $\mathrm{PbZrO}_{3}$ presumably results in a contraction of the Brillouin zone compared to $\mathrm{PbTiO}_{3}$, potentially impacting the phonon energy spectra and Umklapp scattering rates. Additionally, the reduction of symmetry due to the tilted octahedra could serve as an additional source of disorder in the material that is capable of scattering phonons. The glass-like trend in thermal conductivity for an orthorhombic crystal is not unique to $\mathrm{PbZrO}_{3}$ as other perovskites such as sodium niobate $\left(\mathrm{NaNbO}_{3}\right)$ exhibit similar characteristics. [22] The potential causes for the discrepancy between $\mathrm{PbTiO}_{3}$ and $\mathrm{PbZrO}_{3}$ will be discussed in greater detail later in this chapter.

It is interesting to note that a similar discontinuity in the thermal conductivity across the MPB also appears in the bulk data reported in Refs. [25, 26] where $\kappa$ decreases by roughly $26.5 \%$ within the vicinity of the MPB (the specific compositions for the PKR7M and PKR-8 materials in Ref. [25] are not reported; only that they correspond to the rhombohedral and tetragonal sides of the MPB, respectively). The discontinuity in the thin film data is present in both of the finer composition sets $(0.42<x<0.54)$ fired at pyrolysis temperatures of $350^{\circ} \mathrm{C}$ and $400^{\circ} \mathrm{C}$, respectively, albeit with slightly different degrees of abruptness. For the $350^{\circ} \mathrm{C}$ samples, the measured value of $\kappa$ decreases by $23 \%$ between $0.48<x<0.51$, while for the $400^{\circ} \mathrm{C}$ samples, $\kappa$ decreases by $20.3 \%$ between $0.51<x<$ 0.54 .

Since the discontinuity appears in all three of the aforementioned data sets spanning fine-grained thin films to bulk samples, this suggests that it is caused by an intrinsic property of the PZT solid-solution in the vicinity of the MPB and speaks to the complex crystallographic landscape near the MPB composition in PZT. The crystal structure of PZT 
compositions both at and adjacent to the MPB has been studied extensively for decades to understand the nature of the transition to/from rhombohedral and tetragonal symmetry, as well as its impact on the piezoelectric, elastic and dielectric properties [21, 92]. In particular, the elastic stiffness has been shown to be extremely sensitive to composition around the MPB, decreasing by $15-35 \%$ at the MPB compared to adjacent tetragonal compositions [92]. Given the direct correlation between elastic moduli and the speed of sound in a material, it is possible that the observed discontinuity in $\kappa$ is due to a decrease in the phonon velocities at and around the MPB compositions. Furthermore, the existence of an intermediate monoclinic phase had been presented [93] and explored relatively recently via a variety of techniques including synchrotron x-ray diffraction [94, 95], raman spectroscopy [96, 97] and neutron powder diffraction [98]. Specifically, [94] reported a significant change in the volume of the unit cell $\left(\approx 135.15 \AA^{3}\right)$ for the monoclinic phase at $x=0.46$ and 0.47 compared to the adjacent rhombohedral $\left(\approx 67.97 \AA^{3}\right)$ and tetragonal $\left(\approx 67.35 \AA^{3}\right)$ regions. Similar to the case with bulk $\mathrm{PbZrO}_{3}$, this increase in unit cell volume may result in a contraction of the Brillouin zone, presumably affecting the phonon energy spectra and Umklapp scattering rates. Additional experiments are required to establish the root cause of the interesting thermal properties around the MPB composition and will be the focus of future work.

\subsection{Semi-Classical Model for Thermal Conductivity of PZT}

To gather insight regarding the overall impact of various phonon scattering mechanisms on the thermal conductivity of PZT solid-solutions, we employ a semi-classical kinetic theory model for phonon thermal conductivity. Based on the observations and conclusions drawn from an assesment of the temperature trends for bulk $\mathrm{PbTiO}_{3}$ and $\mathrm{PbZrO}_{3}$, an alloytype model for the thermal conductivity of PZT as a function of solid-solution composition is developed and presented. 


\subsubsection{Examining trends in bulk $\mathrm{PbTiO}_{3}$ and $\mathrm{PbZrO}_{3}$}

Our first application of this model is to examine the hypothesis that the glass-like thermal conductivity of $\mathrm{PbZrO}_{3}$ is caused by anharmonic scattering effects due to the large unit cell volume compared to $\mathrm{PbTiO}_{3}$. The approach taken here is to use the model to extract the anharmonic (phonon-phonon) scattering coefficients for bulk $\mathrm{PbTiO}_{3}$ from the data from [22] and then scale these coefficients based on the differences in the structural and elastic properties between $\mathrm{PbTiO}_{3}$ and $\mathrm{PbZrO}_{3}$. If the resultant model for $\mathrm{PbZrO}_{3}$ using these scaled coefficients compares well with the data from [23, 24], this will be a strong indication that anharmonic effects are indeed a dominant source of phonon scattering in crystals with glass-like trends over temperature.

Assuming a Debye-type dispersion, the expression for the thermal conductivity from kinetic theory is:

$$
\kappa=\frac{\hbar^{2}}{6 \pi^{2} k_{B} T^{2}} \sum_{j} \int_{0}^{\omega_{c, j}} \tau_{\mathrm{tot}, j} \frac{\exp \left[\frac{\hbar \omega_{j}}{k_{B} T}\right]}{\left(\exp \left[\frac{\hbar \omega_{j}}{k_{B} T}\right]-1\right)^{2}} \frac{\omega_{j}^{4}}{v_{j}} d \omega
$$

where the summation is over the three acoustic phonon modes (one longitudinal, two transverse) and $j$ denotes the particular mode, $\hbar$ is the reduced Planck's constant, $k_{B}$ is the Boltzmann constant, $\omega$ is the phonon angular frequency, $\omega_{c, j}$ is the cutoff frequency, $T$ is the temperature, $v_{j}$ is the phonon group velocity and $\tau_{\text {tot }, j}$ is the phonon scattering time. The cutoff frequency for integration over $\omega$ is determined according to $\omega_{c, j}=\left(2 \pi v_{j}\right) / a$, where $a$ is the lattice parameter for the crystal.

The phonon scattering time, $\tau_{\mathrm{tot}, j}$ in Eq. 4.2 represents the combined contribution of various phonon scattering mechanisms towards the calculated value of $\kappa$. Contributions due to phonon-phonon (Umklapp) scattering $\left(\tau_{\mathrm{u}, j}\right)$, Rayleigh-like point scattering via mass impurities and strain $\left(\tau_{\mathrm{a}, j}\right)$, and scattering at various boundaries $\left(\tau_{\mathrm{b}, j}\right)$ are combined into a single term via Matthiessen's rule [36, 37, 99]: 


$$
\begin{gathered}
\tau_{\mathrm{tot}, j}=\left(\tau_{\mathrm{u}, j}+\tau_{\mathrm{a}, j}+\tau_{\mathrm{b}, j}\right)^{-1} \\
\tau_{\mathrm{u}, j}=\left(B_{j} T \omega_{j}^{2} \exp \left[-\frac{C_{j}}{T}\right]\right)^{-1} \\
\tau_{\mathrm{a}, j}=\left(A_{\mathrm{j}} \omega_{j}^{4}\right)^{-1} \\
\tau_{\mathrm{b}, j}=\left(\frac{v_{j}}{d}\right)^{-1}
\end{gathered}
$$

Equation 4.4 describes the rate of anharmonic phonon-phonon scattering within the material. In general, assuming elastic scattering, a phonon-phonon interaction can result in two types of collisions; one where energy is conserved but momentum is not and another in which both energy and momentum are conserved. The former is termed an Umklapptype event in which the lack of momentum conservation results in a "resistive" phononphonon process that directly impedes heat flow. The latter is termed a Normal-type event where, though the interaction of two phonons may affect the local distribution of phonon energies in the crystal, their collision is not "resistive" like the Umklapp-type interactions. Equation 4.5 captures the influence of Rayleigh-type point scattering of phonons due to differences in the mass of impurity atoms or vacancies, as well as local strain caused by differences in atomic radii. These effects are particularly strong for the high frequency, short wavelength phonons due to the $\omega^{4}$ dependence. Equation 4.6 describes the scattering time associated with a planar boundary present over a certain length scale, $d$. Examples of this type of scattering include effects due to grain and film boundaries.

The constants $A_{j}, B_{j}$ and $C_{j}$ in Eq. 4.4 and 4.5 are material-specific properties that are determined via fitting the model to measured temperature-dependent data, ideally from bulk single-crystals; in this case, bulk data for $\mathrm{PbTiO}_{3}$ from [22]. When fitting the bulk data, the impact of boundary scattering $\left(\tau_{\mathrm{b}, j}\right)$ is assumed negligible and omitted from Eq. 4.3 . 


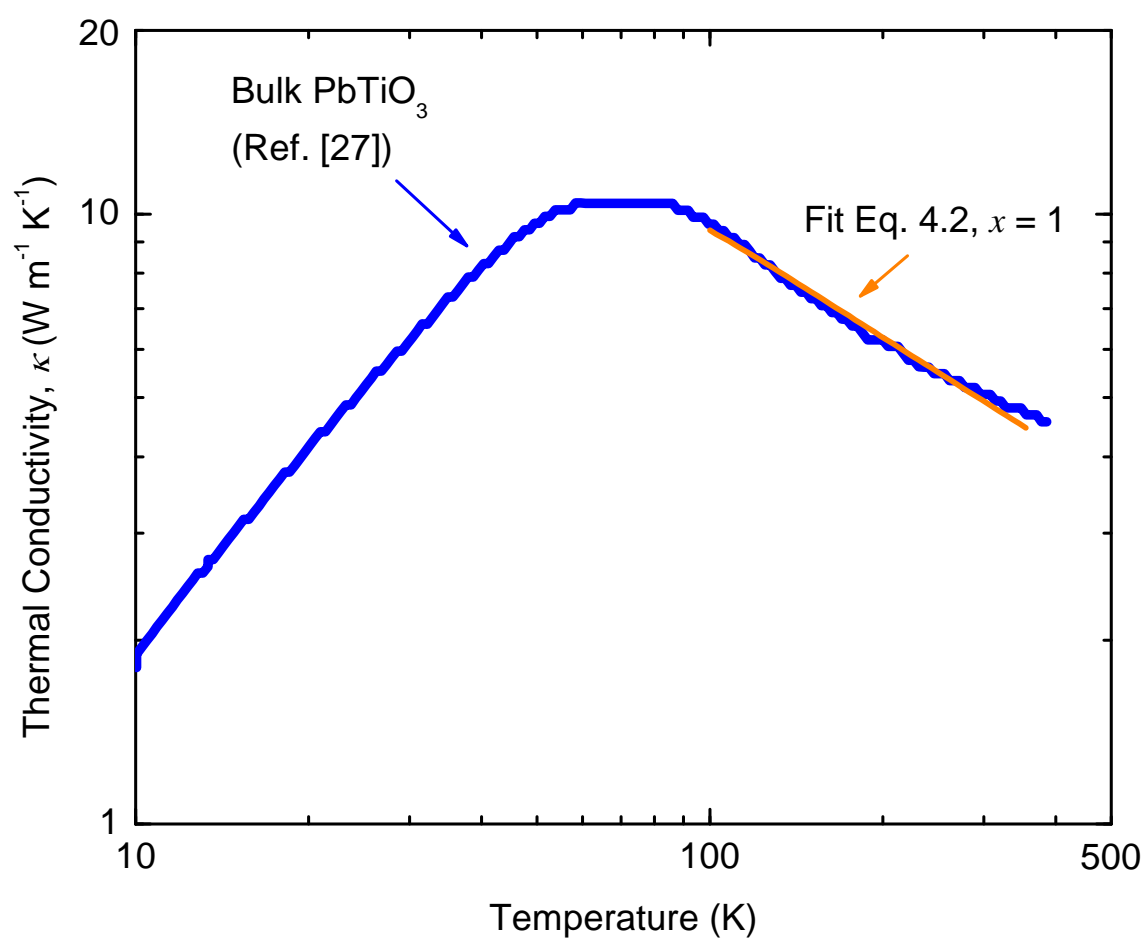

Figure 4.7: Bulk thermal conductivity data for $\mathrm{PbTiO}_{3}$ from [22] used to fit Eq. 4.2] in determining the value of the intrinsic scattering constants $\left(A_{\mathrm{LA}, \mathrm{PTO}}, A_{\mathrm{TA}, \mathrm{PTO}}, B_{\mathrm{LA}, \mathrm{PTO}}, B_{\mathrm{TA}, \mathrm{PTO}}, C_{\mathrm{LA}, \mathrm{PTO}}, C_{\mathrm{TA}, \mathrm{PTO}}\right)$ for the material.

This is reasonable because of the fact that we will be fitting this model to the data over temperatures above the maximum thermal conductivity around $80 \mathrm{~K}$, beyond which $\tau_{\mathrm{tot}, j}$ is likely dominated by $\tau_{\mathfrak{u}, j}$ and $\tau_{\mathrm{a}, j}$ as more high-frequency, short wavelength phonons constitute the heat flux.

The parameters for $\mathrm{PbTiO}_{3}$ listed in Eq. 4.2 through 4.6 that are used in the model are listed in Table 4.2. The model was fit to the data via a least-squares fitting routing for six individual variables; three related to the longitudinal acoustic (LA) phonons and three for the transverse acoustic (TA) phonons. The bulk data for $\mathrm{PbTiO}_{3}$ is plotted in Fig. 4.7. along with the fit of Eq. 4.2 to the data over $100-355 \mathrm{~K}$. Upon fitting the model to the data, the following values were obtained; $A_{\mathrm{LA}, \mathrm{PTO}}=6.4 \times 10^{-42} \mathrm{~s}^{3}, A_{\mathrm{TA}, \mathrm{PTO}}=14 \times 10^{-42}$ 
$\mathrm{s}^{3}, B_{\mathrm{LA}, \mathrm{PTO}}=2.308 \times 10^{-18} \mathrm{~s} \mathrm{~K}^{-1}, B_{\mathrm{TA}, \mathrm{PTO}}=0.880 \times 10^{-18} \mathrm{~s} \mathrm{~K}^{-1}, C_{\mathrm{LA}, \mathrm{PTO}}=156.6 \mathrm{~K}$, $C_{\mathrm{TA}, \mathrm{PTO}}=109.2 \mathrm{~K}$.

Having determined the scattering coefficients for $\mathrm{PbTiO}_{3}$, we next look to apply and adapt these parameters towards a model for $\mathrm{PbZrO}_{3}$ to examine the anharmonicity hypothesis. We make the following assumptions regarding the values of these constants for $\mathrm{PbZrO}_{3}$; we assume that $A_{\mathrm{LA}, \mathrm{PZO}}, A_{\mathrm{TA}, \mathrm{PZO}}, C_{\mathrm{LA}, \mathrm{PZO}}$ and $C_{\mathrm{TA}, \mathrm{PZO}}$ are all equivalent to the values fit for $\mathrm{PbTiO}_{3}$ on the basis that (a) the type and density of point defects are likely to be relatively similar between the two materials, meaning that the values for $A_{j}$ are likely similar and (b) the Debye temperatures of the materials are also quite similar (Table 4.2), meaning that $C_{j}$ is also likely to be similar. We also assume that the values for $B_{j, \mathrm{PZO}}$ can be estimated by scaling the fitted values for $\mathrm{PbTiO}_{3}$ in an effort to approximate the increase in anharmonicity, and thus phonon-phonon scattering in $\mathrm{PbZrO}_{3}$ relative to $\mathrm{PbTiO}_{3}$.

We start by considering the expression for resistive (Umklapp) phonon-phonon scattering processes $\left(B_{j}\right)$ presented in Ref. [100]

$$
B_{j}=\frac{\hbar^{2} \gamma_{j}^{2}}{M v_{j}^{2} \theta}
$$

where $\hbar$ is the reduced Planck's constant, $\gamma_{j}$ is the mode-specific Grüneisen parameter, $M$ is the average atomic mass of the material, $v_{j}$ is the mode-specific sound velocity and $\theta_{\mathrm{D}}$ is the Debye temperature. Using Eq.4.7, we can determine a scaling factor based on the ratio of the calculated values of $B_{j, \mathrm{PZO}}$ and $B_{j, \mathrm{PTO}}$ :

$$
\frac{B_{j, \mathrm{PZO}}}{B_{j, \mathrm{PTO}}}=\left(\frac{\gamma_{j, \mathrm{PZO}}}{\gamma_{j, \mathrm{PTO}}}\right)^{2}\left(\frac{M_{\mathrm{PTO}}}{M_{\mathrm{PZO}}}\right)\left(\frac{v_{j, \mathrm{PTO}}}{v_{j, \mathrm{PZO}}}\right)^{2}\left(\frac{\theta_{\mathrm{D}, \mathrm{PTO}}}{\theta_{\mathrm{D}, \mathrm{PZO}}}\right)
$$

The values for $\gamma_{j, \mathrm{PZO}}$ and $\gamma_{j, \mathrm{PZO}}$ are estimated according to the related solid properties [99]:

$$
\gamma_{\mathrm{LA}}=\frac{3|\beta| M^{2}}{C_{v}} ; \gamma_{\mathrm{TA}}=\frac{3|\beta| G^{2}}{C_{v}}
$$


where $|\beta|$ is the absolute value of the linear expansion coefficient, $M$ and $G$ are the longitudinal and shear elastic moduli, respectively, and $C_{v}$ is the volumetric heat capacity. Using the values listed in Table 4.2, we calculate the scaling factor for both the LA and TA modes to be:

$$
\frac{B_{\mathrm{LA}, \mathrm{PZO}}}{B_{\mathrm{LA}, \mathrm{PTO}}}=7.361 ; \frac{B_{\mathrm{TA}, \mathrm{PZO}}}{B_{\mathrm{TA}, \mathrm{PTO}}}=5.381
$$

The resulting values for the $\mathrm{PbZrO}_{3}$-related constants in Eq. 4.4 are therefore $B_{\mathrm{LA}, \mathrm{PZO}}=(7.361) B_{\mathrm{LA}, \mathrm{PTO}}=16.99 \times 10^{-18} \mathrm{~s} \mathrm{~K}^{-1}$ and $B_{\mathrm{TA}, \mathrm{PZO}}=(5.381) B_{\mathrm{TA}, \mathrm{PTO}}=4.735$ $\mathrm{x} 10^{-18} \mathrm{~s} \mathrm{~K}^{-1}$.

Of the many parameters that go into the scaling of these coefficients, the two that contribute significantly to the large increases are the values for $\beta$ and $C_{v}$ for the two materials. Through Eq. 4.9, the values of $\beta$ and $C_{v}$ for $\mathrm{PbZrO}_{3}$ result in a larger value of $\gamma_{j}$ compared to $\mathrm{PbTiO}_{3}$, ultimately yielding the large scaling factors. These larger values of $B_{\mathrm{LA}, \mathrm{PZO}}$ and $B_{\mathrm{TA}, \mathrm{PZO}}$ compared to the values for $\mathrm{PbTiO}_{3}$ represent an expected increase in the rate of anharmonic phonon-phonon scattering that may have a significant impact on the thermal conductivity of $\mathrm{PbZrO}_{3}$.

Figure 4.8 plots the bulk data for $\mathrm{PbTiO}_{3}$ and $\mathrm{PbZrO}_{3}$ from Figure 4.5 along with the models calculated via Eq. 4.2 using the aforementioned scattering coefficients either fit or derived for each material. Despite the large increase in the $B_{j}$ coefficients, the model for $\mathrm{PbZrO}_{3}$ (magenta dashed line) fails to capture the trend of the $\mathrm{PbZrO}_{3}$ data. While the increased rate of anharmonic scattering does in fact decrease the magnitude of the thermal conductivity, yielding values that are comparable to the data around $350 \mathrm{~K}$, the negative slope over the range $80-400 \mathrm{~K}$ is all wrong as the data shows $\kappa$ slowly increasing over this range. Given the temperature and $\omega^{2}$ dependence contained in Eq. 4.4, the thought had been that a sufficiently-large increase in the $B_{j, \mathrm{PZO}}$ scattering coefficient could result in a dramatic reduction in $\kappa$ even at lower temperatures, ultimately leading to glass-like 


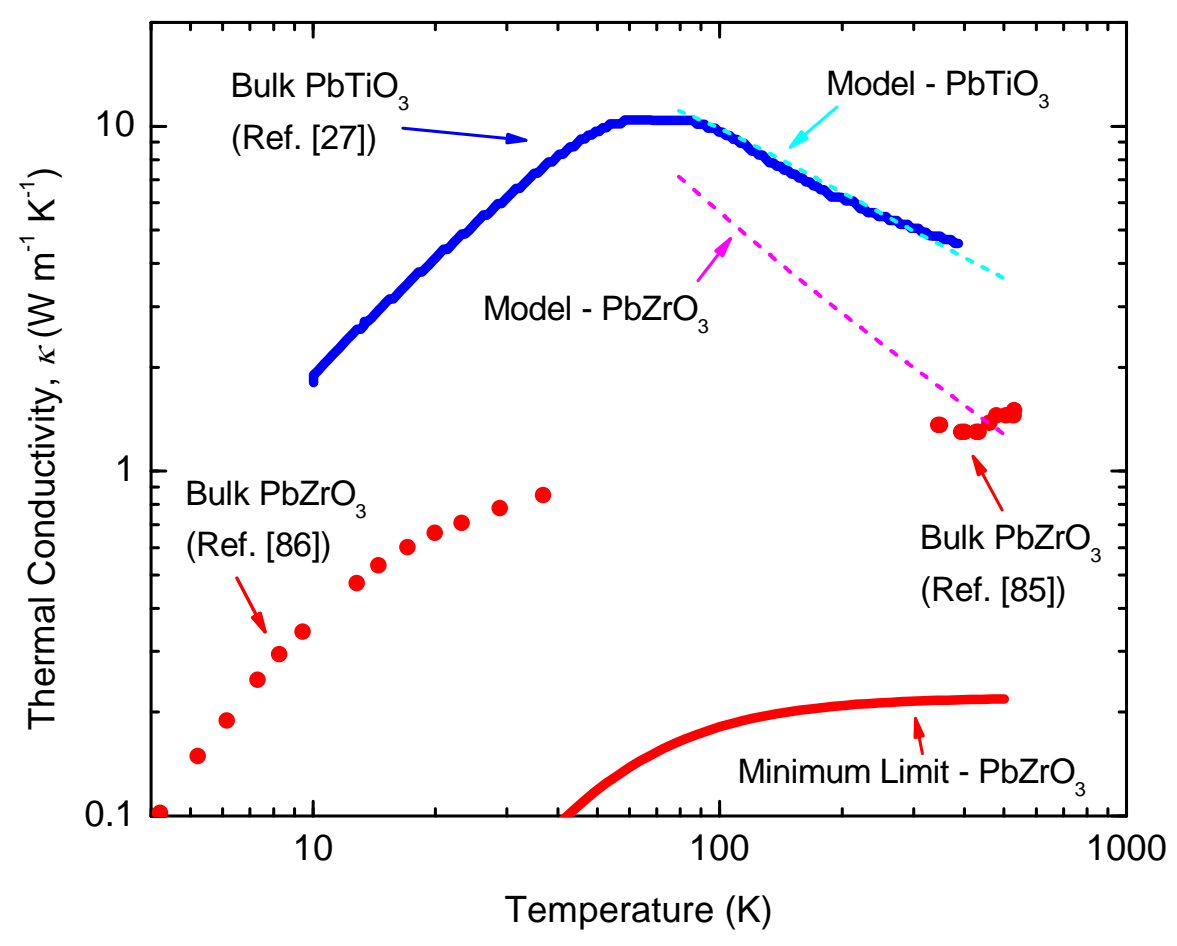

Figure 4.8: Bulk thermal conductivity data for $\mathrm{PbTiO}_{3}$ from [22] and $\mathrm{PbZrO}_{3}$ from [23, 24], along with calculated models for each material using Eq. 4.2. Also plotted is the calculated minimum limit for $\mathrm{PbZrO}_{3}$ using Eq. 4.11]in Eq.4.2.

temperature trends. Based on these results, even the aggressive scaling of the anharmonic scattering term explored here cannot explain the $\mathrm{PbZrO}_{3}$ data on its own.

To provide some context regarding the $\mathrm{PbZrO}_{3}$ data before moving forward, the calculated minimum limit for thermal conductivity [10] for $\mathrm{PbZrO}_{3}$ is also plotted in Figure 4.8 (red line). This minimum limit model is also calculated using Eq. 4.2, though $\tau_{\text {tot }, j}$ is replaced by

$$
\tau_{\min , j}=\left(\frac{\omega_{j}}{\pi}\right)^{-1}
$$

which defines the minimum scattering time for the phonons in the system to be one-half 
the period of oscillation between adjacent atoms (in short, phonon scattering at every atom in the material). This model has been used to examine thermal transport in a variety of heavily-disordered material systems where the concept of phonons being scattered at every atom may be valid [10].

As expected, the minimum limit lies well below the measured $\kappa$ for $\mathrm{PbZrO}_{3}$, but it is important to note the similarity in temperature trends between the $\mathrm{PbZrO}_{3}$ data and minimum limit model. Ultimately, this comparison highlights the fact that the scattering times (and therefore, the mean free paths) must be extremely short to yield the glass-like temper-

ature trends observed in these materials. We will utilize these observations in the following section concerning the model for the thermal conductivity of PZT as a function of composition, as well as the results in Chapter 5 for bilayer PZT heterostructures.

\subsubsection{Thermal conductivity of $\operatorname{PbZr}_{1-x} \mathrm{Ti}_{x} \mathrm{O}_{3}$ as a function of $x$}

This section details the development of a model for the thermal conductivity of lead zirconate titanate $\left(\mathrm{PbZr}_{1-x} \mathrm{Ti}_{x} \mathrm{O}_{3}, \mathrm{PZT}\right)$ thin films as a function of composition, $x$. Assuming a Debye-type dispersion, the expression for the thermal conductivity as a function of $x$ is:

$$
\kappa(x)=\frac{\hbar^{2}}{6 \pi^{2} k_{B} T^{2}} \sum_{j} \int_{0}^{\omega_{c, j}(x)} \tau_{\mathrm{tot}, j}(x) \frac{\exp \left[\frac{\hbar \omega_{j}}{k_{B} T}\right]}{\left(\exp \left[\frac{\hbar \omega_{j}}{k_{B} T}\right]-1\right)^{2}} \frac{\omega_{j}^{4}}{v_{j}(x)} d \omega
$$

which is identical to Eq. 4.2 except that several parameters are now a function of $x$. This model is based on the consideration of the solid-solution as a virtual crystal where the inputs to the model are composite values of the appropriate fraction of each constitutive material. Therefore, inputs such as $v_{j}(x)$ and $a(x)$ are calculated via:

$$
\begin{gathered}
v_{j}(x)=\left[(1-x) v_{j, \mathrm{PZO}}^{-2}+x v_{j, \mathrm{PTO}}^{-2}\right]^{-1 / 2} \\
a(x)=(1-x) a_{\mathrm{PZO}}+x a_{\mathrm{PTO}}
\end{gathered}
$$


where the lattice parameters $a_{\mathrm{PZO}}$ and $a_{\mathrm{PTO}}$ are the cube root of their respective unit cell volumes. Additionally, the cutoff frequency for integration over $\omega$ is determined according to $\omega_{c, j}(x)=\left(2 \pi v_{j}\right) / a(x)$, where $a(x)$ is the lattice parameter. It should be noted here that this model attempts to include the significant difference in unit cell volume between $\mathrm{PbZrO}_{3}$ and $\mathrm{PbTiO}_{3}$ primarily though the calculated value of $a(x)$ and its impact on the cutoff frequency, $\omega_{c, j}(x)$.

The phonon scattering time, $\tau_{\text {tot }, j}(x)$ in Eq. 4.2 is also a function of composition, $x$, and represents the combined contribution of various phonon scattering mechanisms towards the calculated value of $\kappa(x)$. Contributions due to Rayleigh-like point scattering via mass impurities and strain $\left(\tau_{\mathrm{a}}(x)\right)$, and scattering at various boundaries $\left(\tau_{\mathrm{b}, j}(x)\right)$ are combined into a single term via Matthiessen's rule [36] with similar virtual-crystal considerations applied to each term [78-80, 99]. Scattering due to anharmonic phonon-phonon scattering is omitted from this model due to the earlier observation that these effects are not a dominant source of phonon scattering in $\mathrm{PbZrO}_{3}$. Alternatively, this model is built on the assumption that $\tau_{\mathrm{a}}(x)$ and $\tau_{\mathrm{b}, j}(x)$ are best suited to describe the phonon scattering processes in this system. In this model for $\kappa(x)$ in PZT, these expressions take the following form:

$$
\begin{gathered}
\tau_{\mathrm{tot}, j}(x)=\left(\tau_{\mathrm{a}}(x)+\tau_{\mathrm{b}, j}(x)\right)^{-1} \\
\tau_{\mathrm{a}}(x)=\left(x(1-x) A \omega_{j}^{4}\right)^{-1} \\
\tau_{\mathrm{b}, j}(x)=\left(\frac{v_{j}(x)}{d}\right)^{-1}
\end{gathered}
$$

Equation 4.16 describes the influence of various types of point-scattering mechanisms, including scattering due to differences in the mass of the substitutional atoms $(\mathrm{Zr}, \mathrm{Ti})$ and scattering due to local strain caused by differences in the atomic radii of the substitutional 
atoms. This expression differs from Eq. 4.5 in the inclusion of a $x(1-x)$ prefactor due to the virtual crystal/mixture considerations in heavily alloyed systems [78, 79, 101-103]. Equation 4.17 describes the scattering time associated with a planar boundary present over a certain length scale, $d$, including features such as grain and film boundaries.

Similar to the work on $\mathrm{Si}_{x} \mathrm{Ge}_{1-x}$ in [79, 103], a value for the scattering constant $A$ in Eq. 4.16 was required. Ideally, the value of $A$ is determined by fitting the model to temperature dependent data for one of the heavily-alloyed compositions (i.e., $0.2<x<0.8$ ). Given the lack of temperature dependent data at most compositions for PZT, measurements were made from $78-400 \mathrm{~K}$ on the $30 / 70 \mathrm{PZT}$ thin film $(x=0.7)$, pyrolyzed at $350^{\circ} \mathrm{C}$, from this study.

The results of these measurements are plotted in Figure 4.9 along with bulk data and the minimum limit for bulk $\mathrm{PbZrO}_{3}$. The data for the the 30/70 PZT thin film exhibits a glass-like trend in thermal conductivity as a function of temperature, similar to the $\mathrm{PbZrO}_{3}$ data from [23, 24]. It appears that the thermal conductivity for the thin film data begins to roll-off/decrease at comparatively higher temperatures than the $\mathrm{PbZrO}_{3}$ data, possibly indicating a significant difference in the scattering processes between the two samples.

The model defined by Eqs. 4.12 through 4.17 was fit to both the 30/70 PZT thin film ( $x$ $=0.7)$ and the bulk $\mathrm{PbZrO}_{3}(x=0)$ data using a least-squares fitting routing, treating $A$ and $d$ as adjustable parameters. Fitting the $\mathrm{PbZrO}_{3}$ data results in $A_{\mathrm{PZO}}=20.5 \times 10^{-42} \mathrm{~s}^{3}$ and $d_{\mathrm{PZO}}=91 \mathrm{~nm}$, while fitting the 30/70 PZT thin film data results in $A_{30 / 70}=3.922 \times 10^{-42}$ $\mathrm{s}^{3}$ and $d_{30 / 70}=4.61 \mathrm{~nm}$. It is interesting to note that there are considerable differences in the values of both parameters between the two data sets. First, the value of $d_{30 / 70}$ is over an order of magnitude smaller than $d_{\mathrm{PZO}}$, presumably related to the large differences in thermal conductivity at low temperatures. Second, the value of $A_{\mathrm{PZO}}$ is approximately five times larger than $A_{30 / 70}$ due to the fact that the $\mathrm{PbZrO}_{3}$ data begins to flatten out at lower temperatures where there are not as many higher-frequency phonons comprising the phonon flux.

All together, a comparison of the data and fitted values seem to indicate that the phonon 


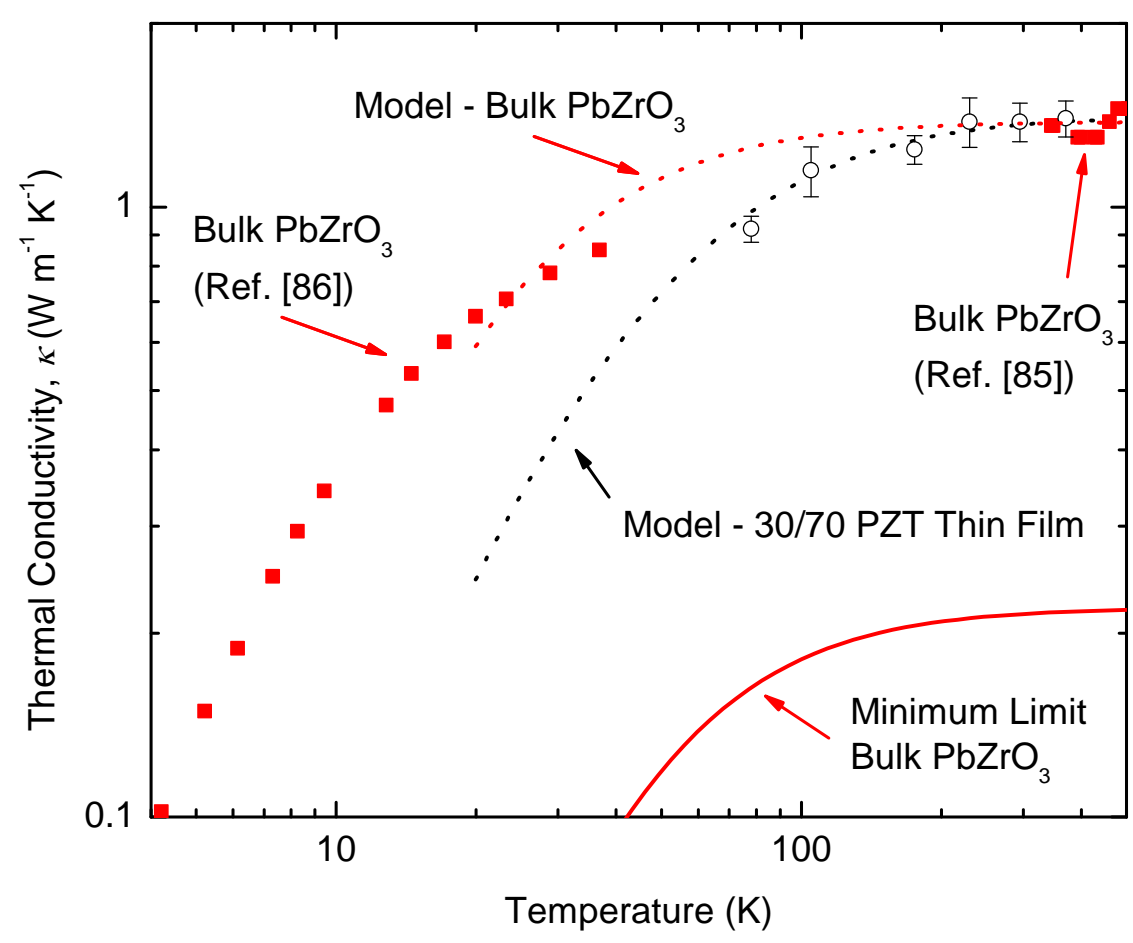

Figure 4.9: Bulk thermal conductivity data for $\mathrm{PbZrO}_{3}$ from [23, 24] and measured values for 30/70 PZT thin films ( $x=0.7,325 \mathrm{~nm}$ thick) over temperature. Also plotted is the calculated minimum limit for $\mathrm{PbZrO}_{3}$ using Eq. 4.11 in Eq. 4.12 with $x=0$. The models represent fits to the data defined by Eqs. 4.12 through 4.17, treating $A$ and $d$ as adjustable parameters to gain insight regarding the phonon scattering processes in these samples.

scattering rates in the 30/70 PZT thin film samples are significantly higher than the bulk $\mathrm{PbZrO}_{3}$ samples at low temperatures, whereas the scattering rates at higher temperatures are quite similar, resulting in similar thermal conductivities. One potential cause for this difference at low temperatures is the fact that the 30/70 PZT sample is a thin film, and therefore may have additional phonon scattering at the film boundary described by Eq. 4.17. However, given the extremely small values of $d_{30 / 70}(=4.61 \mathrm{~nm})$ fit with this model compared to the various boundary-like length scales associated with this sample (film thickness of $325 \mathrm{~nm}$; average grain size of $95 \mathrm{~nm}$ ), it appears that the impact of these length scales is likely negligible. To confirm this, the 30/70 PZT thin film data was fit once again for $A$ and 
$d_{30 / 70}$ with the inclusion of two additional boundary scattering terms (Eq. 4.17 ) in the total scattering time expression of Eq. 4.15 with $d_{\text {film }}=325 \mathrm{~nm}$ and $d_{\text {grain }}=95 \mathrm{~nm}$. The result was that the fitted values for $A$ and $d_{30 / 70}$ changed by less than $1 \%$, indicating that neither the film nor grain boundaries are the cause of the large difference between the samples.

An alternative explanation could come from the domain structure of the 30/70 PZT thin film. In their work on $\mathrm{BaTiO}_{3}$ single crystals [13], Mante and Volger observed a similar difference in the thermal conductivities of their unpoled and actively-poled samples at low temperatures. They attribute this difference to phonon scattering at ferroelastic domain walls with the following argument; in the unpoled state, there are many domain walls that scatter phonons and result in a lower thermal conductivity and when the number of these domain walls decreases significantly while actively poled, the thermal conductivity jumps up at these lower temperatures due to the decrease in phonon scattering rates.

While both the $\mathrm{PbZrO}_{3}$ and the 30/70 PZT thin film likely contain a large number of ferroelastic domain walls, the domain-periodicity (spacing from one wall to another) may be significantly different. Several works have examined the ferroelastic domain structures in similar single-layer 30/70 PZT thin films [104-108] via plan-view piezoresponse force microscopy (PFM), estimating a domain-periodicity of approximately 20-30 nm [104]. Given that this potential length scale for ferroelastic domain walls is shorter than the physical dimensions of the film and its grains, it very well could be the source of the decrease in thermal conductivity at low temperatures in the 30/70 PZT thin film, particulary compared to bulk $\mathrm{PbZrO}_{3}$ depending on its domain-periodicity.

Using the fit values for $A$ and $d$ from the 30/70 PZT thin film data in the expression for $\tau_{\mathrm{tot}, j}(x)$ (Eq. 4.3), we can then calculate the model for $\kappa(x)$ in Eq. 4.12 and compare it to the thin film PZT data. The model is calculated for all compositions ranging from 0 $\leq x \leq 1$, and the relevant inputs for $\mathrm{PbZrO}_{3}$ and $\mathrm{PbTiO}_{3}$ for the virtual crystal are listed in Table 4.2. The intent of the model is to capture the general trend for the PZT solidsolution system over the compositional range. The extension of this model to include the discontinuity around the MPB will be the focus of future work. 


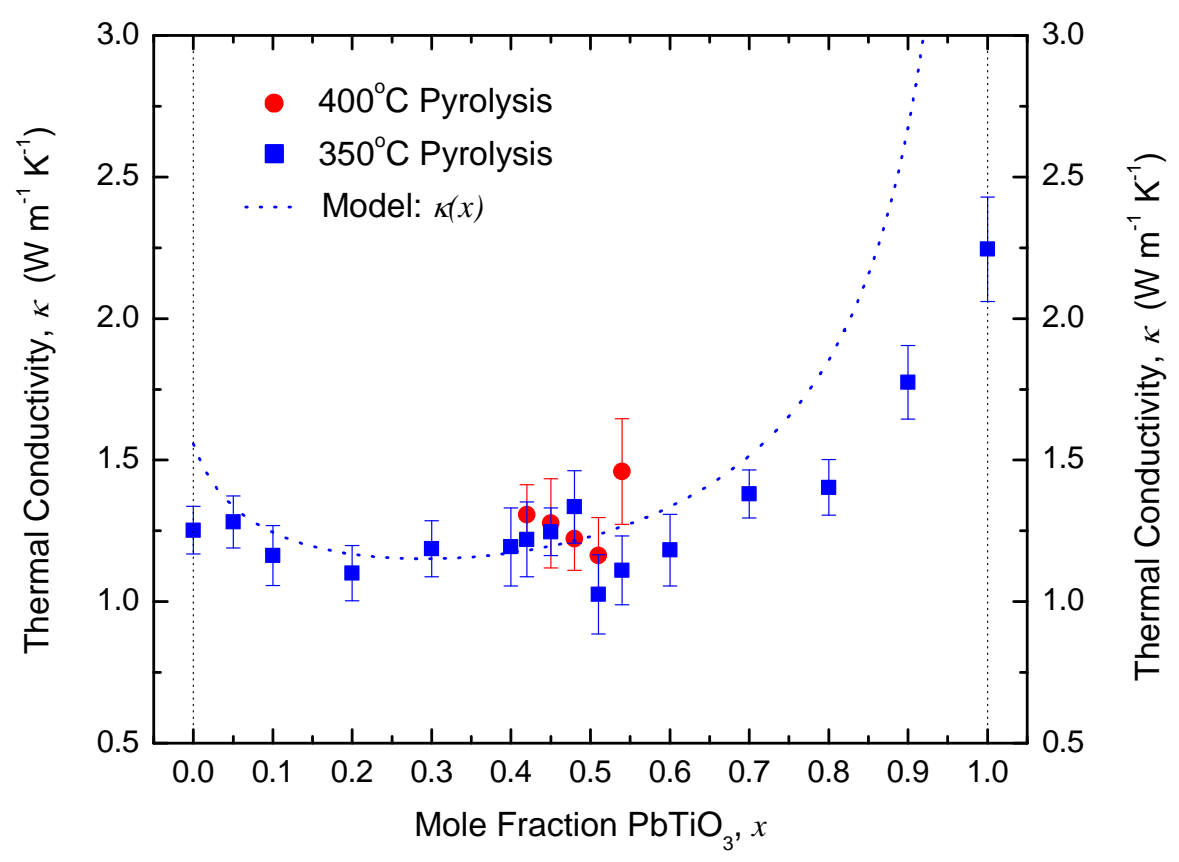

Figure 4.10: Thermal conductivity of PZT thin films plotted with the model from Eq. 4.12. The data and model related to samples pyrolyzed at $350^{\circ} \mathrm{C}$ are denoted by blue squares and dashed blue line, respectively, while the data for samples pyrolyzed at $400^{\circ} \mathrm{C}$ are denoted by red circles.

Figure 4.10 plots the model along with the thin film PZT data. The use of $A$ and $d$ from the temperature-dependent 30/70 PZT thin film data results in good agreement to the data over much of the compositional range. It should be noted that while the model does not include any anharmonic phonon-phonon scattering effects, the model agrees quite well with the data as $x \rightarrow 0$. This fact further supports the observations from the temperature trends for $\mathrm{PbZrO}_{3}$ that anharmonic phonon-phonon scattering effects are weak compared to other scattering rates in the material system for these compositions. Conversely, the model and data deviate from one another as $x \rightarrow 1$ with the model over predicting the thermal conductivity, highlighting that importance of anharmonic effects as the material composition approaches phase-pure $\mathrm{PbTiO}_{3}$. In general, the model compares quite well to the experimental data and appears to capture the trend as a function of $x$, apart from the MPB region. 


\subsection{Summary}

This chapter introduced the lead zirconate titanate $\left(\mathrm{PbZr}_{1-x} \mathrm{Ti}_{x} \mathrm{O}_{3}, \mathrm{PZT}\right)$ solid solution material system, highlighting its technological importance to several large industries. The versatility of the material comes from the ability to vary the composition, $x$, and tailor its properties to suit a given application (ferroelectric, piezoelectric, etc). Surprisingly, relatively little is known about the nature of thermal transport in such an important material. Therefore, we have completed a comprehensive study of the thermal conductivity of PZT thin films across the compositional phase diagram from $0<x<1$. As part of the study, we also investigated the large difference in bulk thermal conductivity reported in the literature between $\mathrm{PbZrO}_{3}$ and $\mathrm{PbTiO}_{3}$, leading to the fabrication and measurement of our own bulk $\mathrm{PbZrO}_{3}$ sample as well as bulk PZT samples at two compositions. Based on our analysis using a semi-classical model for the thermal conductivity, we postulate that the glass-like trend in thermal conductivity observed for $\mathrm{PbZrO}_{3}$ is not due to an increase in anharmonic phonon-phonon scattering associated with the large unit cell volume of $\mathrm{PbZrO}_{3}$ compared to $\mathrm{PbTiO}_{3}$. Instead, the glass-like trends are likely caused by a hitherto unidentified source of disorder in crystalline $\mathrm{PbZrO}_{3}$ that results in high phonon scattering rates, even at low temperatures. Additionally, a model for the thermal conductivity of PZT as a function of composition $(\kappa(x))$ is developed and presented. Using temperature dependent measurements of the 30/70 PZT thin film $(x=0.7)$ to extract the necessary scattering constants, the resulting model agrees quite well with the experimental data for compositions $0<x<$ 0.8. For compositions $0.8<x<1$, the model begins to deviate from the experimental data, over predicting the value of the thermal conductivity. This discrepancy for compositions approaching $x=1$ is due to the fact that the model does not include anharmonic phonon scattering effects, highlighting the importance of these scattering processes as the material composition approaches phase-pure $\mathrm{PbTiO}_{3}$. 
Table 4.2: Material properties for $\mathrm{PbZrO}_{3}$ and $\mathrm{PbTiO}_{3}$ used in the model for $\kappa(x)(\mathrm{Eq} .4 .2$ )

\begin{tabular}{|c|c|c|}
\hline \multicolumn{3}{|l|}{$\mathrm{PbZrO}_{3}$} \\
\hline Crystal Structure & Orthorhombic & Ref. [109] \\
\hline Unit Cell Dimensions ( $a \times b \times c, \AA)$ & $5.87 \times 11.74 \times 8.20$ & Ref. [109] \\
\hline Formula Units per Unit Cell & 8 & Ref. [109] \\
\hline Density $\left(\rho_{\text {PZO }}, \mathrm{g} \mathrm{cm}^{-3}\right)$ & 7.98 & Ref. [110] \\
\hline Longitudinal Elastic Modulus $\left(M_{\mathrm{PZO}}, \mathrm{GPa}\right)$ & 190 & Ref. [111] \\
\hline Shear Elastic Modulus $\left(G_{\mathrm{PZO}}, \mathrm{GPa}\right)$ & 59 & Ref. [111] \\
\hline Bulk Modulus $\left(E_{\mathrm{PZO}}, \mathrm{GPa}\right)$ & 111.33 & Calc. \\
\hline Longitudinal Acoustic Velocity $\left(v_{\mathrm{LA}}, \mathrm{m} \mathrm{s}^{-1}\right)$ & 4928 & Ref. [111] \\
\hline Transverse Acoustic Velocity $\left(v_{\mathrm{TA}}, \mathrm{m} \mathrm{s}^{-1}\right)$ & 2746 & Ref. [111] \\
\hline Unit Cell Volume $\left(V_{\mathrm{PZO}}, \mathrm{nm}^{3}\right)$ & 0.5651 & Calc. \\
\hline Atomic Density $\left(n_{\mathrm{PZO}}, \mathrm{nm}^{-3}\right)$ & 69.36 & Calc. \\
\hline Volumetric Heat Capacity $\left(C_{v, \mathrm{PZO}}, \mathrm{J} \mathrm{cm} \mathrm{cm}^{-3} \mathrm{~K}^{-3}\right)$ & 2.56 & Ref. [88] \\
\hline Linear Thermal Expansion $\left(\beta_{\mathrm{PZO}}, \mathrm{K}^{-1}\right)$ & $9.2 \times 10^{-6}$ & Ref. [112] \\
\hline Debye Temperature $\left(\theta_{\mathrm{D}}, \mathrm{K}\right)$ & 391 & Ref. [23] \\
\hline Average Atomic Mass $\left(M_{\mathrm{A}}, \mathrm{amu}\right)$ & 69.285 & Calc. \\
\hline \multicolumn{3}{|l|}{$\mathrm{PbTiO}_{3}$} \\
\hline Crystal Structure & Tetragonal & Ref. [113] \\
\hline Unit Cell Dimensions ( $a \times b \times c, \AA)$ & $3.91 \times 3.91 \times 4.14$ & Ref. [113, 114] \\
\hline Formula Units per Unit Cell & 1 & Ref. [113] \\
\hline Density $\left(\rho_{\mathrm{PTO}}, \mathrm{g} \mathrm{cm}^{-3}\right)$ & $7.40-7.80$ & Ref. [115] \\
\hline Longitudinal Elastic Modulus ( $\left.M_{\mathrm{PTO}}, \mathrm{GPa}\right)$ & 240 & Ref. [116] \\
\hline Shear Elastic Modulus $\left(G_{\mathrm{PTO}}, \mathrm{GPa}\right)$ & 92.0 & Ref. [116] \\
\hline Bulk Modulus $\left(E_{\mathrm{PTO}}, \mathrm{GPa}\right)$ & 86.9 & Calc. \\
\hline Longitudinal Acoustic Velocity $\left(v_{\mathrm{LA}}, \mathrm{m} \mathrm{s}^{-1}\right)$ & 5066 & Ref. [117] \\
\hline Transverse Acoustic Velocity $\left(v_{\mathrm{TA}}, \mathrm{m} \mathrm{s}^{-1}\right)$ & 3300 & Ref. [117] \\
\hline Unit Cell Volume $\left(V_{\mathrm{PTO}}, \mathrm{nm}^{3}\right)$ & 0.0633 & Calc. \\
\hline Atomic Density $\left(n_{\mathrm{PTO}}, \mathrm{nm}^{-3}\right)$ & 78.48 & Calc. \\
\hline Volumetric Heat Capacity $\left(C_{v, \mathrm{PTO}}, \mathrm{J} \mathrm{cm} \mathrm{cm}^{-3} \mathrm{~K}^{-3}\right)$ & 2.72 & Ref. [88] \\
\hline Linear Thermal Expansion $\left(\beta_{\mathrm{PTO}}, \mathrm{K}^{-1}\right)$ & $-3.3 \times 10^{-6}$ & Ref. [118] \\
\hline Debye Temperature $\left(\theta_{\mathrm{PTO}}, \mathrm{K}\right)$ & 394 & Ref. [119] \\
\hline Average Atomic Mass ( $\left.M_{\mathrm{PTO}}, \mathrm{amu}\right)$ & 60.61 & Calc. \\
\hline
\end{tabular}




\section{Chapter 5}

\section{Phononic Thermal Switch via PZT}

\section{Bilayer Structure}

As in the $\mathrm{BiFeO}_{3}$ thin films studied in [16], PZT thin films of various compositions exhibit ferroelastic domain structures which are clamped, and therefore immobile, due to strain interactions with the bulk substrate that they are deposited on. In order to create mobile domain walls that would be able to respond to external stimuli, bilayer PZT heterostructures were employed as these configurations have been shown to result in domain structures that are easily susceptible to applied electric fields [27, 28]. In previous works, Anbusathaiah et al. $([27,[28])$ had shown that in bilayer structures comprised of a tetragonal $\mathrm{PbZr}_{0.3} \mathrm{Ti}_{0.7} \mathrm{O}_{3}$ layer $(x=0.7)$ on top of a rhombohedral $\mathrm{PbZr}_{0.7} \mathrm{Ti}_{0.3} \mathrm{O}_{3}$ layer $(x=0.3)$, there is a complex ferroelastic domain structure that exists only in the top tetragonal layer and is able to reversibly and repeatably respond to an applied electric field. The explanation given for the observed mobility of the domain structure in the top thin film layer is that the rhombohedral layer screens the strain interaction between the top film and the bulk substrate, resulting in untethered domain walls that are able to move much more freely and the energy landscape changes due to applied electric fields. Interestingly, the response of the domain structure within the top layer of the heterostructure to an applied electric field is to nucleate more domain walls, which is contrary to domain wall behavior in bulk ferroelec- 
tric materials where domain wall density decreases with applied fields to promote uniform polarization of the ferroelectric material. This increase in domain wall density with applied electric fields was confirmed in the works by Anbusathaiah et al. through piezoresponse force microscopy (PFM) as well as X-ray diffraction (XRD) patterns as separate means to monitor the changes in domain structure.

This chapter chronicles our work to create a phonon thermal switch via the modulation of the ferroelastic domain wall density in bilayer PZT thin films using applied electric fields. Based on the earlier work by Anbusathaiah et al. [27, 28], we have fabricated several bilayer PZT heterostructures of our own and have characterized the change in domain wall density while under applied electric fields via two different plan-view imaging methods [12]. Using two different types of electrical probing stations integrated into our TDTR system, we measure the room temperature and temperature dependent thermal conductivity of these bilayer PZT heterostructures as a function of applied electric field with the goal of understanding the nature of phonon thermal transport in these phononic thermal devices.

\subsection{Bilayer PZT Thermal Switch: Room Temperature}

The first step was to make room temperature TDTR measurements of the bilayer PZT heterostructures as a function of applied electric field strength. This section provides details regarding sample fabrication and characterization, as well as details for the analysis of the thermal data.

\subsubsection{Sample Fabrication \& Characterization}

Bilayer PZT heterostructures similar to those in Refs. [27, 28] were fabricated via chemical solution deposition (CSD) on platinized-silicon substrates at Sandia National Laboratories to investigate whether these mobile ferroelastic domains could be utilized to demonstrate thermal switching. Figures 5.1 (a) illustrates the bilayer PZT device geometry created to facilitate the successful measurement via TDTR of tunable thermal trans- 


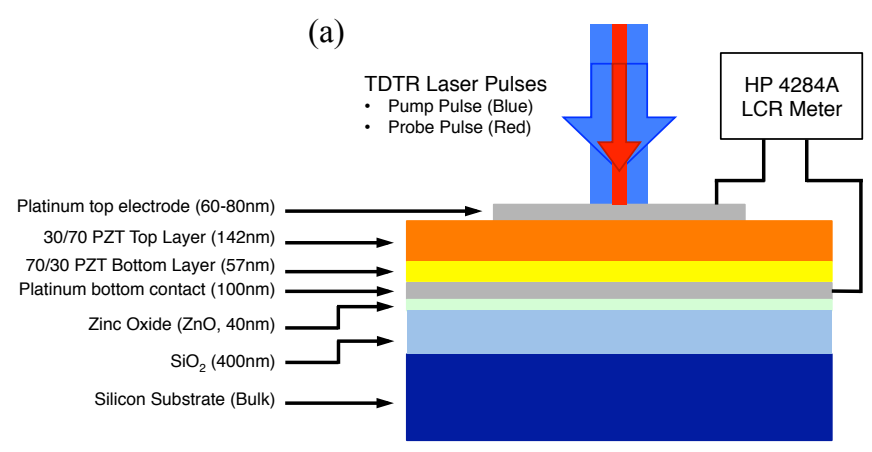

(b)

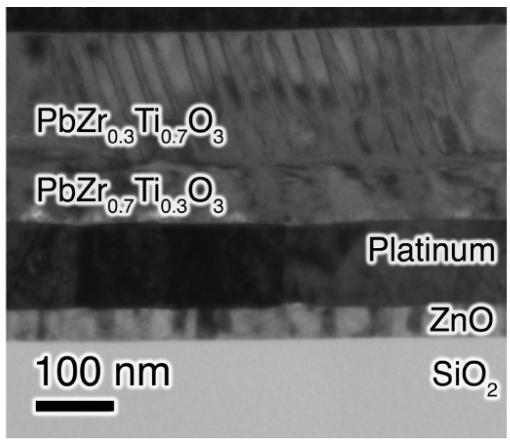

Figure 5.1: (a) Experimental geometry showing cross section of PZT bilayer sample. (b) Crosssection bright-field transmission electron micrograph (TEM) of a PZT bilayer film showing a stripe pattern of $90^{\circ}$ ferroelastic domains in the upper $\mathrm{PbZr}_{0.3} \mathrm{Ti}_{0.7} \mathrm{O}_{3}$ layer.

port within the structure. Polycrystalline lead zirconate titanate (PZT) bilayer thin films composed of a tetragonal symmetry PZT layer $\left(\mathrm{PbZr}_{0.3} \mathrm{Ti}_{0.7} \mathrm{O}_{3}\right)$ on top of a rhombohedral symmetry PZT layer $\left(\mathrm{PbZr}_{0.7} \mathrm{Ti}_{0.3} \mathrm{O}_{3}\right)$ were used in this study as these have previously been shown to possess highly mobile ferroelastic domain walls with sub-100 $\mathrm{nm}$ spacing. [27] Films were deposited onto platinized silicon wafers (100 nm Pt/40 nm ZnO/400 nm SiO2/Si (001)) with $\mathrm{PbZr}_{0.3} \mathrm{Ti}_{0.7} \mathrm{O}_{3}$ thicknesses of $142 \pm 3 \mathrm{~nm}$ and $\mathrm{PbZr}_{0.7} \mathrm{Ti}_{0.3} \mathrm{O}_{3}$ thicknesses of $57 \pm 4 \mathrm{~nm}$, as determined using transmission electron microscopy (TEM). Figure 5.1 (b) is a cross-section bright-field transmission electron microscopy image (TEM) of the device structure in which the ferroelastic domain walls (stripes) are clearly visible in the tetragonal $\mathrm{PbZr}_{0.3} \mathrm{Ti}_{0.7} \mathrm{O}_{3}$ top layer. The films were randomly oriented as assessed by X-ray diffraction and clearly showed field-dependent polarization hysteresis at room temperature. Following deposition of the rhombohedral and tetragonal PZT layers, platinum electrodes were deposited on the top film to create metal-insulator-metal structures to facilitate application of the electric field across the PZT bilayer.

\subsubsection{Thermal Measurements \& Analysis}

The data from each TDTR scan is fit to a multilayer thermal model [120-122] via a least-squares fitting routine to extract values for the parameters of interest, including the 
thermal conductivity of different layers and the thermal boundary conductance between layers. To do so accurately, the heat capacities and thicknesses of each layer must be known as inputs for the model. The heat capacities of platinum and silicon at room temperature were taken from the literature [87, 123] as are their thermal conductivities (accounting for the reduction in thermal conductivity of the platinum thin film) [124-126]. For the 30/70 and 70/30 PZT layers, the heat capacities were calculated as a stoichiometric average of the heat capacities of $\mathrm{PbZrO}_{3}$ and $\mathrm{PbTiO}_{3}$ taken from the literature [88]. To validate the calculated heat capacity for PZT compositions using this method, our calculated heat capacity for $30 / 70$ PZT is within $2 \%$ of the value reported in [127]. The thickness of the platinum electrodes was measured via profilometry (Veeco Dektak 8, calibrated on a $48 \mathrm{~nm}$ standard to $\pm 1 \mathrm{~nm}$ ) and the thicknesses of the 30/70 and 70/30 PZT layers were determined from energy dispersive spectroscopy (EDS) maps collected during STEM imaging.

Figure 5.2 shows the sensitivity to various parameters for the four-layer thermal model used in this experiment, as described in [128]. The sensitivities shown correspond to a modulation frequency of $2.57 \mathrm{MHz}$, which was chosen to minimize the sensitivity to the thermal boundary conductance between the top platinum electrode and the 30/70 PZT top layer $\left(h_{\mathrm{K}, 1}\right)$ while providing increased sensitivity to the thermal conductivity of the $30 / 70$ PZT top layer $\left(\kappa_{2}\right)$ between 100-2000 ps of time delay (additional details concerning the selection of thermal modulation frequencies for measurements on bilayer PZT heterostructure can be found in Appendix A). However, this modulation frequency results in a thermal penetration depth of approximately $260 \mathrm{~nm}$, thereby causing the model to also be somewhat sensitive to the thermal conductivity of the 70/30 PZT bottom layer (trace $\kappa_{3}$ ), as well as the effective thermal conductivity of the substrate layers beneath the bottom Pt electrode $\left(\operatorname{trace} \kappa_{4}\right)$.

To address this, a separate 'calibration' sample of 70/30 PZT was deposited via CSD on a similar platinized-silicon substrate to enable determination of the thermal conductivity of the 70/30 PZT and the backside conductance between this layer and the platinized stack below. The thickness of this separate 70/30 PZT layer was determined via cross-sectional 


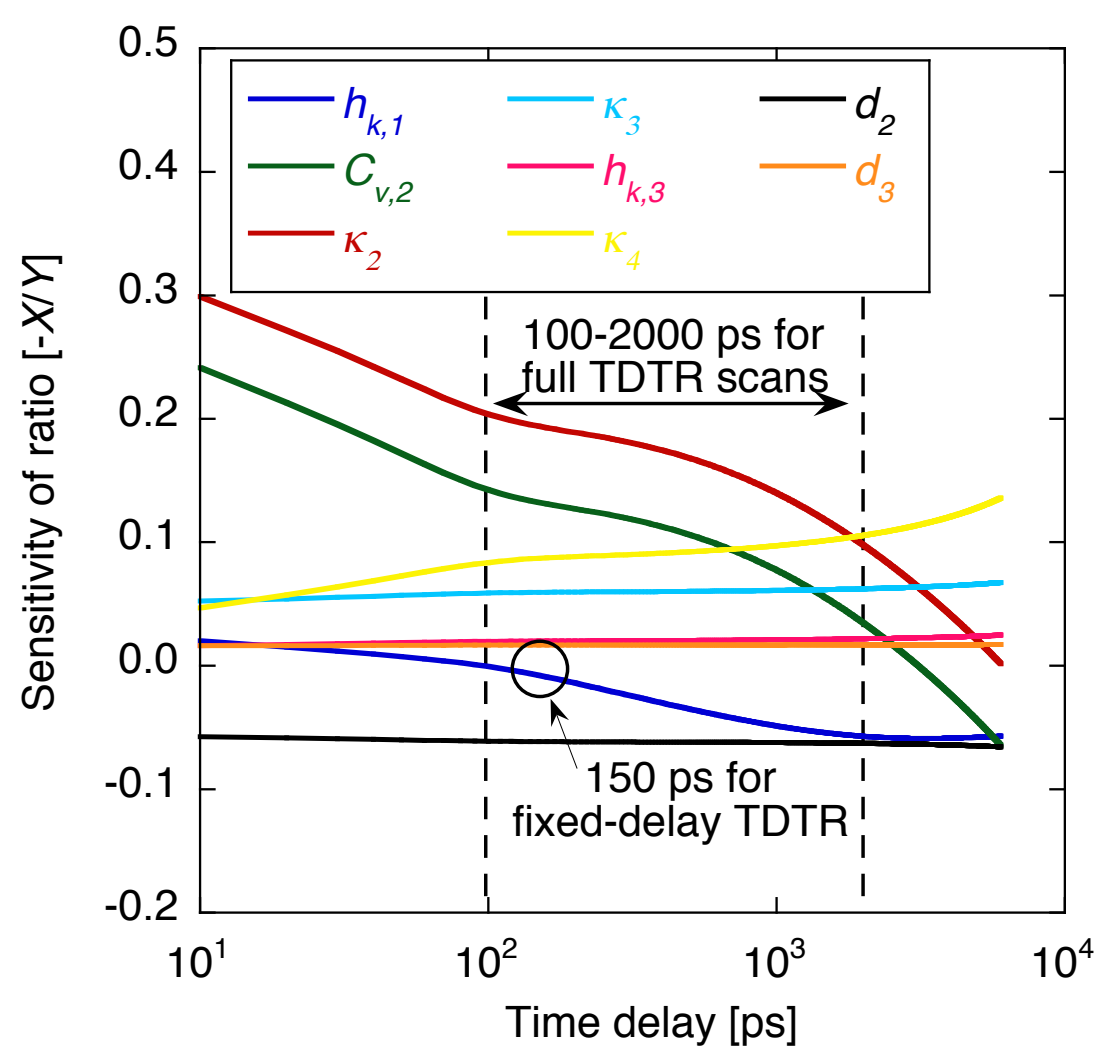

Figure 5.2: Sensitivity of the four-layer thermal model to various parameters at a modulation frequency of $2.57 \mathrm{MHz} . h_{\mathrm{k}, 1}$ is the Kapitza conductance between layers 1 and 2; $C_{\mathrm{v} 2}$ is the heat capacity of layer $2(30 / 70 \mathrm{PZT}) ; \kappa_{2}$ and $\kappa_{3}$ are the thermal conductivities of layer 2 and layer 3 (70/30 PZT), respectively; $h_{\mathrm{k}, 3}$ is the Kapitza conductance between layer 3 and the remaining layers beneath.

SEM to be $80 \pm 2 \mathrm{~nm}$, and the thickness of the platinum electrodes was measured to be 81 $\pm 2 \mathrm{~nm}$ via profilometry. Three TDTR scans were performed on each of three different electrodes at a modulation frequency of $8.8 \mathrm{MHz}$, resulting in a thermal penetration depth of $160 \mathrm{~nm}$. The data was fit to a four-layer model, where layer one was the platinum top electrode $\left(C_{\mathrm{V}}=2.84 \mathrm{~J} \mathrm{~m}^{-3} \mathrm{~K}^{-1}, \kappa=33.8 \mathrm{~W} \mathrm{~m}^{-1} \mathrm{~K}^{-1}, \mathrm{~d}=81 \mathrm{~nm}\right)$, layer two was the 70/30 $\operatorname{PZT}\left(C_{\mathrm{v}}=2.616 \mathrm{~J} \mathrm{~m}^{-3} \mathrm{~K}^{-1}, \mathrm{~d}=80 \mathrm{~nm}\right)$ and layer four was $\mathrm{SiO}_{2}\left(C_{\mathrm{v}}=1.92 \mathrm{~J} \mathrm{~m}^{-3} \mathrm{~K}^{-1}, \kappa=\right.$ $1.4 \mathrm{~W} \mathrm{~m}^{-1} \mathrm{~K}^{-1}, \mathrm{~d}=1 \mathrm{~m}$ (bulk)). Layer three was set as an effective conductivity calculated by adding the thermal resistances of the platinum bottom electrode $\left(\kappa=33.8 \mathrm{~W} \mathrm{~m}^{-1} \mathrm{~K}^{-1}\right.$, $\mathrm{d}=100 \mathrm{~nm}$ ), the interface resistance between the platinum and $\mathrm{ZnO}$ (estimated to be 100 
$\left.\mathrm{MW} \mathrm{m}^{-2} \mathrm{~K}^{-1}\right)$ and the resistance of the $\mathrm{ZnO}$ layer $\left(\kappa=4.5 \mathrm{~W} \mathrm{~m}^{-1} \mathrm{~K}^{-1}\right.$ from [129], $\mathrm{d}=$ $40 \mathrm{~nm}$ ) via a series resistor model. This calculation results in an effective conductivity for layer three of $6.3 \mathrm{~W} \mathrm{~m}^{-1} \mathrm{~K}^{-1}$, and $C_{\mathrm{v}}$ was set to $2.85 \mathrm{~J} \mathrm{~m}^{-3} \mathrm{~K}^{-1}$ as the heat capacities of platinum and $\mathrm{ZnO}$ are nearly equivalent at room temperature. The interface conductance between the 70/30 PZT film and the bottom platinum electrode $\left(h_{\mathrm{K}, 2}\right)$ was set equal to that between the top platinum electrode and the 70/30 PZT film $\left(h_{\mathrm{K}, 1}\right)$ as these interfaces are chemically equivalent. The fitted data determined the thermal conductivity of the 70/30 PZT layer to be $2.01 \pm 0.057 \mathrm{~W} \mathrm{~m}^{-1} \mathrm{~K}^{-1}$ and the interface conductance between the 70/30 PZT and either electrode to be approx. $90 \mathrm{MW} \mathrm{m}^{-2} \mathrm{~K}^{-1}$.

With the thermal conductivity of the 70/30 PZT bottom layer and thermal boundary conductance between this layer and the platinum electrode now known, TDTR measurements were conducted on the bilayer samples to determine the change in thermal conductivity of the 30/70 PZT top layer due to an applied electric field. Figure 5.1 (a) is a diagram depicting the sample geometry and measurement techniques applied to each device, and Table 5.1 summarizes the fixed values used in the four-layer model when fitting the TDTR data taken on the bilayer samples while under applied electric fields.

Table 5.1: Fixed values employed or the 4-layer model when fitting the TDTR data acquired from the PZT bilayer samples while under applied electric fields.

\begin{tabular}{cccccc}
\hline \hline Layer & Description & $d(\mathrm{~nm})$ & $C_{\mathrm{v}}\left(\mathrm{J} \mathrm{m}^{-3} \mathrm{~K}^{-1}\right)$ & $\kappa\left(\mathrm{W} \mathrm{m}^{-1} \mathrm{~K}^{-1}\right)$ & $h_{\mathrm{k}}\left(\mathrm{MW} \mathrm{m}^{-2} \mathrm{~K}^{-1}\right)$ \\
\hline$(1)$ & Pt electrode & $62 \pm 2$ & 2.840 & 33.8 & - \\
- & Pt:30/70 & - & - & - & 90 \\
$(2)$ & $30 / 70 \mathrm{PZT}$ & $142 \pm 3$ & 2.707 & - & - \\
- & $30 / 70: 70 / 30$ & - & - & - & 1000 \\
$(3)$ & $70 / 30 \mathrm{PZT}$ & $57 \pm 4$ & 2.616 & 2.01 & - \\
- & $70 / 30: \mathrm{Pt}$ & - & - & - & 90 \\
$(4)$ & Substrate & Bulk & 1.920 & 2.3 & - \\
\hline \hline
\end{tabular}

Measurements were made on several different devices both before any bias fields were applied (the unpoled, or virgin domain state), as well as over a range of applied biases 
( $\max \pm 10 \mathrm{~V})$. Five TDTR scans were performed at each applied field condition to provide an assessment of uncertainty in the measurement. For each device, the laser spot was focused onto the top surface of the platinum top electrode and was left in the same spot for all of the applied field conditions. This reduced the uncertainty due to variations in the thickness of the platinum top electrode that would need to be included had the measurement location changed on every scan. The $1 / e^{2}$ radii of the pump and probe beams when focused on the sample surface were measured via a beam profiler (Thorlabs, BP104-UV) and are approximately $14 \mu \mathrm{m}$ and $6 \mu \mathrm{m}$, respectively. Note that this is significantly larger than the average grain size of the sample measured and therefore represents that many grains are measured at once. As mentioned previously, the pump beam was sinusoidally modulated at $2.57 \mathrm{MHz}$ using an electro-optic modulator to minimize our sensitivity to $h_{\mathrm{K}, 1}$ relative to the thermal conductivity of the 30/70 PZT top layer (See Fig. 5.2).

The optical power delivered to the sample by both the pump and probe beams was measured using a thermopile-based power meter (Newport 919P series), and varied between 7-14 $\mathrm{mW}$ for either beam on any given measurement period. There are two separate temperature rises within the area of the laser spot due to the optical power delivered to the sample; one is a DC (or steady-state) temperature rise due to both the pump and probe, while the second is an AC temperature rise associated with the modulated pump beam. Details concerning the calculations of these temperature rises can be found in [120] and [1]. As a worst-case assessment, we calculate these temperatures for pump and probe powers of $14 \mathrm{~mW}$ at the $1 / e^{2}$ spot sizes mentioned earlier, assuming an absorptivity of $15 \%$ in platinum at $800 \mathrm{~nm}$ and $55 \%$ at $400 \mathrm{~nm}$. We calculate a maximum steady-state temperature rise of 1.75 - $2 \mathrm{~K}$ due to the absorption of DC pump and probe power. Additionally, we calculate a maximum $\mathrm{AC}$ temperature rise due to the $2.57 \mathrm{MHz}$ sinusoidally modulated pump beam of between $1.7-2.4 \mathrm{~K}$, assuming that the thermal conductivity of the $30 / 70$ PZT top layer is somewhere between $1-2 \mathrm{~W} \mathrm{~m}^{-1} \mathrm{~K}^{-1}$. Therefore, the maximum overall temperature rise in the sampled area during the measurement is between $3.45-4.4 \mathrm{~K}$.

DC electric fields were applied to the bilayer devices using an LCR meter and the room 
temperature probe station integrated into the TDTR system. Before making electrical contact, base-line measurements were made of each device in the unpoled, virgin domain state. Following this, contact was made and confirmed via the measurement of the capacitance of the device using the LCR meter at a measurement frequency of $1 \mathrm{kHz}$ and an AC smallsignal measurement amplitude of $100 \mathrm{mV}$ RMS. This AC field applied across the device ( $4.6 \mathrm{kV} / \mathrm{cm}$ RMS) is not expected to result in any significant electrocaloric effects [130] in the PZT at a drive frequency of $1 \mathrm{kHz}$ and at room temperature operation (far from any phase transitions in either part of the bilayer). To confirm this, different small signal values up to $1 \mathrm{~V}$ RMS ( $46 \mathrm{kV} / \mathrm{cm}$ RMS) were also applied during measurement and no differences in the TDTR signals or calculated thermal conductivity could be observed. Bias voltages of up to $\pm 10 \mathrm{~V}$ in magnitude were applied in $2.5 \mathrm{~V}$ increments, resulting in maximum electric fields of approx. $460 \mathrm{kV} / \mathrm{cm}$. The loss tangent was monitored during the application of the bias to the film and did not exceed 0.03 during the test, indicating that the sample was stable during the measurement and no breakdown events occurred. Additionally, measurements were made with the DC bias set to $0 \mathrm{~V}$ after applying maximum field with both polarities to compare the thermal conductivities of the 30/70 PZT top layer in both the unpoled, virgin states and the poled, remanant states.

The TDTR data collected under the applied electric fields were then fit to the aforementioned multilayer thermal model to determine the thermal conductivity of the 30/70 PZT top layer $\left(\kappa_{2}\right)$. Based on the measurements made on the 70/30 PZT calibration sample, the thermal conductivity of layer three is set to $2.01 \mathrm{~W} \mathrm{~m}^{-1} \mathrm{~K}^{-1}$ for $70 / 30 \mathrm{PZT}$ and the interface conductances between the platinum electrodes and adjacent PZT layers $\left(h_{\mathrm{K}, 1}, h_{\mathrm{K}, 2}\right)$ are both set to $90 \mathrm{MW} \mathrm{m}^{-2} \mathrm{~K}^{-1}$. Additionally, we assume that the thermal boundary conductance between the 30/70 top and 70/30 bottom PZT layers $\left(h_{\mathrm{K}, 2}\right)$ is essentially infinite $\left(>1 \mathrm{GW} \mathrm{m}^{-2} \mathrm{~K}^{-1}\right)$. While we expect that this is not the case in actuality, this assumption is made because we are unable to definitively characterize domain structure across the interface between the PZT layers via the techniques available. As a result, any possible effects localized near the interface specifically are lumped into the overall conductivity of 
the 30/70 PZT top layer $\left(\kappa_{2}\right)$. The thermal conductivity of the 30/70 PZT top layer $\left(\kappa_{2}\right)$ and the thermal conductivity of the substrate $\left(\kappa_{4}\right)$ are treated as free parameters when fitting the data with the least-squares algorithm. The thermal conductivity of the substrate must be fit in order to gain agreement between the data and the model and consistently yielded a value of $2.3 \pm 0.2 \mathrm{~W} \mathrm{~m}^{-1} \mathrm{~K}^{-1}$ for all fits of the TDTR data. At our modulation frequency, the TDTR measurements do not probe below the $\mathrm{SiO}_{2}$ layer, so in effect, this $\mathrm{SiO}_{2}$ can be treated as a semi-infinite substrate. Therefore, the effective value for $\kappa_{4}$ determined during the fit represents the total thermal resistance measured from the substrate stack comprised of $100 \mathrm{~nm}$ platinum on $40 \mathrm{~nm} \mathrm{ZnO}$ on an effectively semi-infinite substrate of $\mathrm{SiO}_{2}$, and the $\mathrm{Pt} / \mathrm{ZnO}$ and $\mathrm{ZnO} / \mathrm{SiO}_{2}$ interface resistances. We assume the heat capacity of this final layer to be equal to that of the $\mathrm{SiO}_{2}$ substrate since this effectively semi-infinite layer provides the most thermal mass that is probed in our substrate stack, and our out-of-phase signal is more affected by this layer compared to the other layers in the substrate.

\subsubsection{Results \& Discussion}

Figure 5.3 (a) shows the results for $\kappa_{2}$ versus applied electric field for a bilayer PZT device. The error bars represent the uncertainty in the measurement over five TDTR scans performed at each applied field. For each device, the laser spot was focused onto the top surface of the platinum top electrode and was left in the same spot for all of the applied field conditions. This was done to reduce uncertainty due to variations in the thickness of the platinum top electrode that would need to be included had the measurement location changed on every scan; this resulted in much tighter error bars than a typical TDTR experiment as we alleviate the main source of experimental variation and uncertainty by fixing the measurement location on the sample when applying the electric field.

The thermal conductivity decreases by $11 \%$ from the unpoled, virgin state under an applied field of $475 \mathrm{kV} / \mathrm{cm}$. Increasing the magnitude of the electric field across the device decreases the thermal conductivity of the 30/70 PZT. PFM and SEM analyses of the 30/70 PZT top layer showed that the domain wall density increases when this film is subjected 
(a)

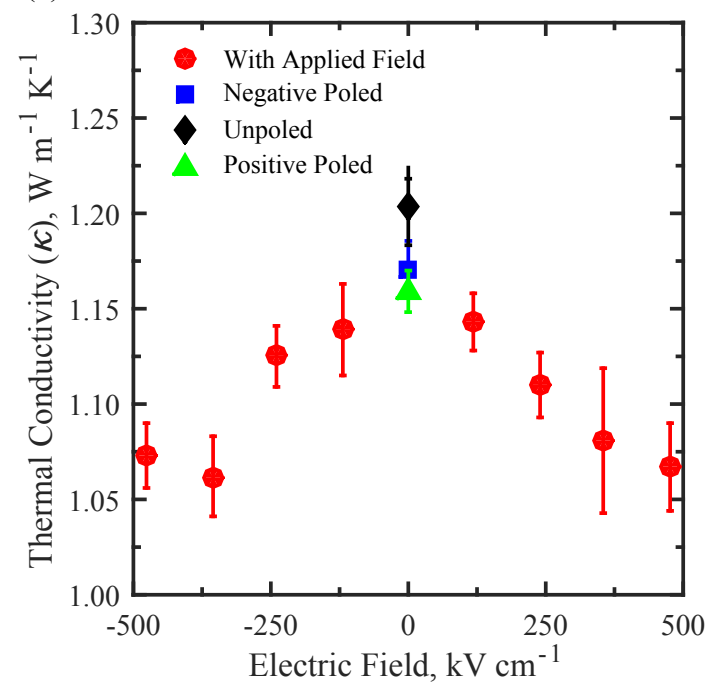

(b)

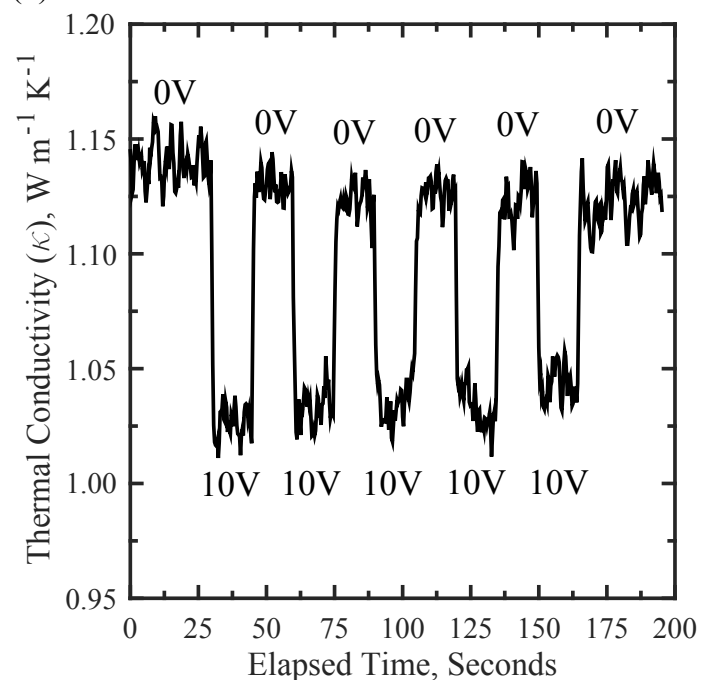

Figure 5.3: (a) Thermal conductivity versus applied electric field for a PZT bilayer device. The thermal conductivity decreases with increasing electric field magnitude due to the increase in domain wall density that impedes phonon transport within the film. [12] (b) Real-time measurement of the thermal conductivity of the PZT bilayer at a fixed TDTR delay time of $150 \mathrm{ps}$ as the applied bias is cycled five times between the no-field and high-field $(10 \mathrm{~V}, 475 \mathrm{kV} / \mathrm{cm})$ conditions. [12]

to an applied electric field. Additionally, it has been shown in single crystalline $\mathrm{BiFeO}_{3}$ films [16] that increased domain wall density reduces the thermal conductivity due to the different numbers of domain-variants. Therefore, the decrease in the thermal conductivity is attributed to an increase in the rate of phonon scattering at domain boundaries when the density of domain walls increases in the presence of an electric field. Furthermore, we see that there is a clear relationship between the thermal conductivity of the film and the applied electric field, highlighting the tunable nature of these devices. It should be noted that the black data point corresponds to the thermal conductivity of the 30/70 PZT top layer in the unpoled, virgin domain state, while the blue and green data points at zero field represent the thermal conductivity when the DC fields were removed following either positive or negative poling at maximum field (approx. $\pm 460 \mathrm{kV} / \mathrm{cm}$ ), which represent the remanent ferroelectric polarization states. The difference between the thermal conductivities in the unpoled versus poled states is believed to be due to the domain configuration that persists when the electric field is removed. 
To further demonstrate the functionality of the thermal device, we monitored the thermoreflectance of the platinum top electrode in real-time as bias was applied and removed (see Figure 5.3 (b)). In this measurement, the probe delay relative to the pump pulse was fixed at 150 ps to minimize the sensitivity of the model to changes in the thermal boundary conductance between the top platinum electrode and the 30/70 PZT top layer relative to the sensitivity of the 30/70 PZT top layer while allowing nearly full diffusion of deposited layer energy through the platinum electrode (i.e., reducing the sensitivity to the thermal conductivity of the platinum). The thermoreflectance of the platinum electrode is directly related to the temperature of the metal. Therefore, for a fixed power deposited on the sample by the laser and resulting heat flux through the sample structure, any changes in the temperature of the top electrode are indicative of changes in the thermal resistance of the multilayer structure beneath it when bias is applied or removed. This allowed for real time observation of the dynamic switching of the thermal conductivity of the 30/70 PZT top layer.

As a final check, we performed the same measurements on a metal-insulator-metal structure with amorphous alumina $\left(a-\mathrm{Al}_{2} \mathrm{O}_{3}, 135 \mathrm{~nm}\right.$ thick $)$ as the insulating layer; a structure in which we do not expect to see any affect on the thermal conductivity under applied electric fields due to the absence of any microstructure in such a disordered film. Figure 5.4 plots the thermoreflectance response of the platinum top electrode in real time at a fixed delay of 150 ps. As in the case of the bilayer sample (Figure 5.3p), the DC field across the sample was switched between $0 \mathrm{kV} / \mathrm{cm}$ and $\pm 370 \mathrm{kV} / \mathrm{cm}$ and held across the structure for approximately $2 \mathrm{~min}$. Unlike the bilayer sample, no change in the thermoreflectance was observed in response to an applied electric field, indicating that the thermal properties of the $a-\mathrm{Al}_{2} \mathrm{O}_{3}$ remain constant under an applied field. This result lends further evidence that the tunability of the domain structure within the PZT bilayer under applied electric fields is the cause of the observed change in the thermal conductivity in our reported results. 


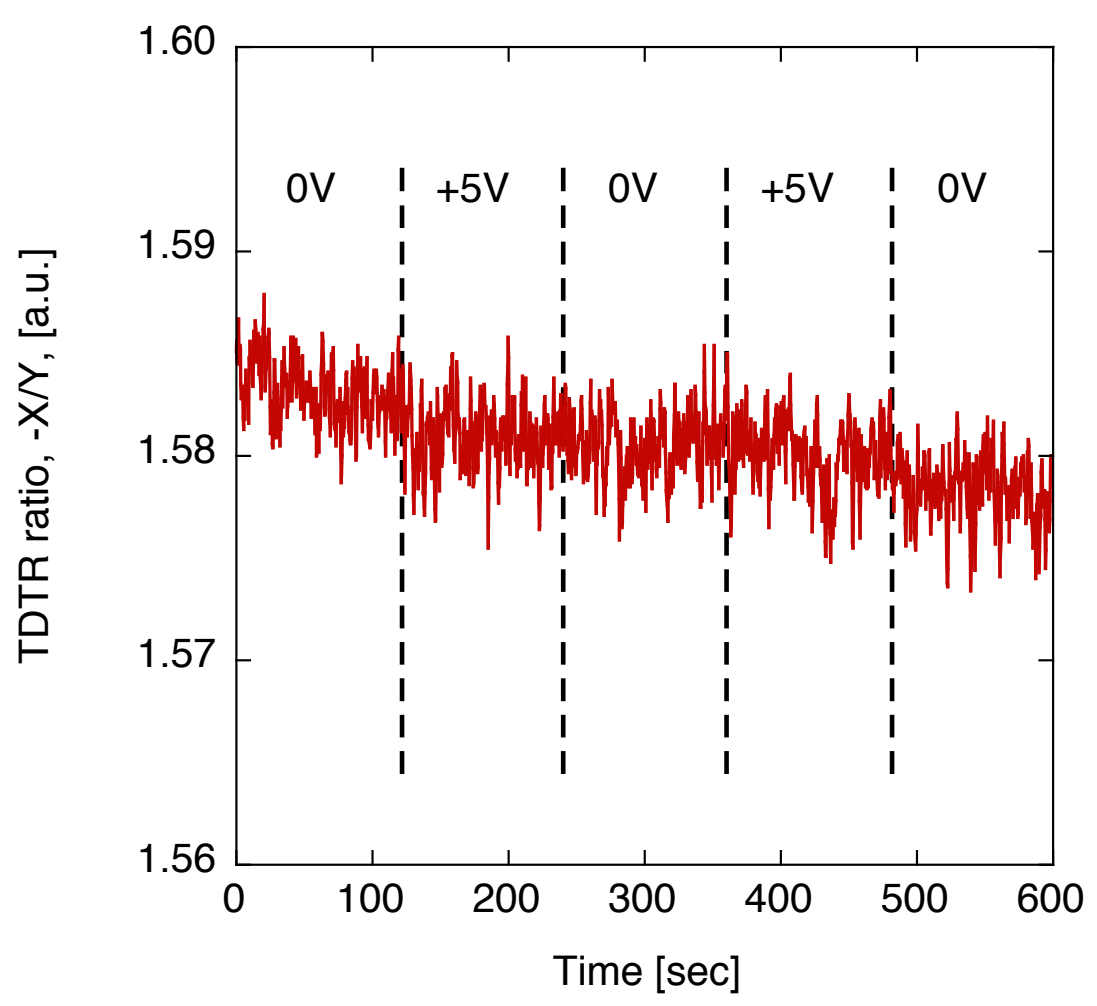

Figure 5.4: Real-time thermoreflectance signal measured via TDTR at a fixed delay of $150 \mathrm{ps}$ for a platinum $/ a-\mathrm{Al}_{2} \mathrm{O}_{3} /$ platinum metal-insulator-metal structure as the applied electric field is cycled between $0 \mathrm{~V}(0 \mathrm{kV} / \mathrm{cm})$ and $\pm 5 \mathrm{~V}( \pm 370 \mathrm{kV} / \mathrm{cm})$. No changes are observed in the thermoreflectance signal, indicating that the response exhibited by the PZT bilayer films is unique to that structure.

\subsection{Bilayer PZT Thermal Switch: Temp. Dependence}

Following the work on room-temperature operation, our next step was to investigate the operation of the phonon thermal switch as a function of temperature. The temperature dependence of the thermal conductivity of a material provides a wealth of insight into the nature of phonon scattering. As the temperature increases, the phonon spectrum is increasingly comprised of high-frequency/short wavelength phonons which are readily scattered by point defects/impurities and fine (10's of $\mathrm{nm}$ ) structure within the material. By inspecting the temperature dependence of the PZT bilayer structure both with and without applied electric fields, we can characterize the impact of the domain structures in the unpoled and poled states on various parts of the phonon spectrum. 


\subsubsection{Sample Fabrication \& Characterization}

(a)

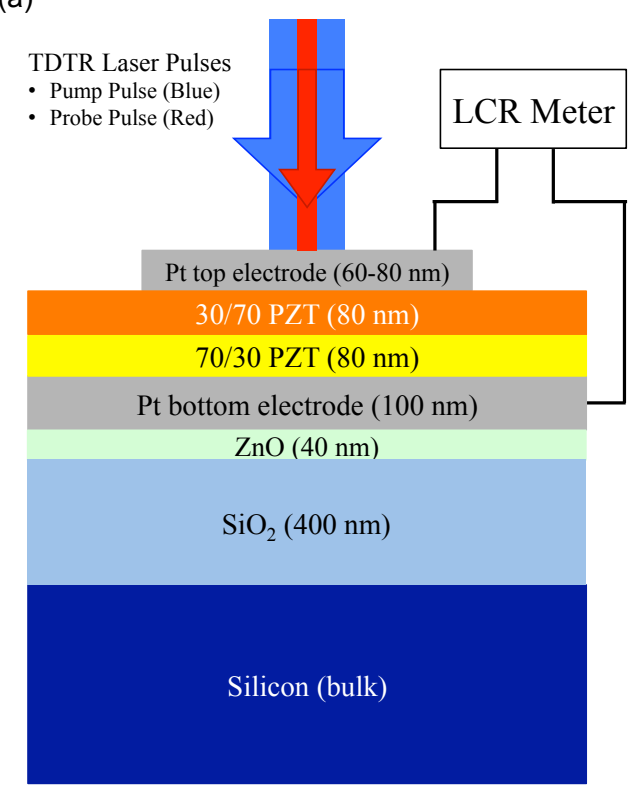

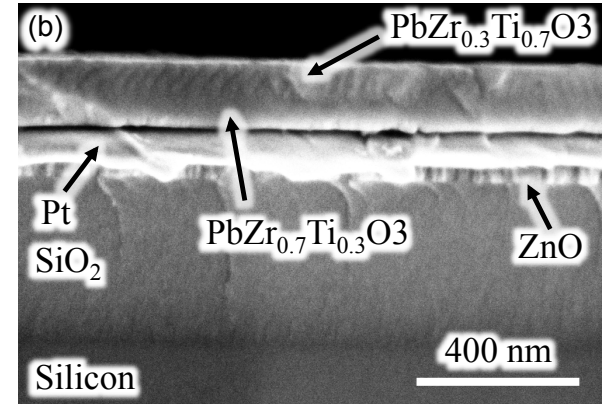



Figure 5.5: (a) Diagram of the 1x1 bilayer PZT heterostructure measured as a function of temperature at zero and $-450 \mathrm{kV} \mathrm{cm}^{-1}$ applied electric fields. (b) Cross-sectional SEM image of the $1 \mathrm{x} 1$ bilayer heterostructure. Note the striped-contrast of the ferroelastic domains in the top tetragonal $\mathrm{PbZr}_{0.3} \mathrm{Ti}_{0.7} \mathrm{O}_{3}$ layer that are not present in the bottom rhombohedral $\mathrm{PbZr}_{0.7} \mathrm{Ti}_{0.3} \mathrm{O}_{3}$ layer. (c) Planview SEM image os the 1x1 bilayer heterostructure, illustrating the anomalously large grain sizes and complex, ultra-fine ferroelastic domain structure of these bilayers. [12, 27, 28]

The PZT bilayer samples that were used for the temperature dependent measurements were similar to those in the room temperature study, comprised of a tetragonal $\mathrm{PbZr}_{0.3} \mathrm{Ti}_{0.7} \mathrm{O}_{3}$ layer (30/70 PZT, $x=0.7$ ) approximately $80 \mathrm{~nm}$ thick on top of a rhombohedral $\mathrm{PbZr}_{0.7} \mathrm{Ti}_{0.3} \mathrm{O}_{3}$ layer (70/30 PZT, $x=0.3$ ) that was also $80 \mathrm{~nm}$ thick, all deposited via CSD on platinizedsilicon substrates. Figure 5.5 (a) shows a diagram of the sample used for these measurements, while Figures 5.5(b) and (c) are cross-section and plan-view SEM images, respectively, of these samples. Due to the fact a single CSD coating step was carried out to deposit the top and bottom layers of this heterostructure, we refer to this sample as a '1x1' PZT bilayer, where the sample from the room temperature study was a '2x1' heterostructure. The $1 \mathrm{x} 1$ geometry was chosen for this study due to the amount of existing 
literature [27, 28, 131, 132] related to similar PZT bilayer configurations.

\subsubsection{Thermal Measurements \& Analysis}



Figure 5.6: Thermal conductivity versus applied electric field measured at room temperature for the 1x1 bilayer PZT device. As observed in the 2x1 PZT heterostructure from [12], the thermal conductivity decreases with increasing electric field magnitude, likely due to the increase in domain wall density that increases the phonon scattering rate within the film.

The TDTR measurements were made with the $1 \mathrm{x} 1$ bilayer sample mounted in the Janis cryogenic probe station, providing the ability to probe the thermal properties of the devices while under an applied electric field over a temperature range of 78 - $400 \mathrm{~K}$. Prior to performing the temperature dependent measurements, the $1 \mathrm{x} 1$ bilayer device was tested at room temperature under applied electric fields of $\pm 500 \mathrm{kV} \mathrm{cm}^{-1}$. Figure 5.6 plots these results for the thermal conductivity of the tetragonal 30/70 PZT top layer as a function of applied electric field. Similar to the $2 \times 1$ bilayer sample measured in [12], the thermal conductivity is found to decrease as the applied electric field of either polarity increases in 
magnitude. When the electric field is removed, the thermal conductivity returns to a value close to that of the unpoled sample. The percent change in the thermal conductivity between the poled and zero field states is only $6 \%$, which is smaller than what was measured in the $2 \times 1$ bilayer device. All in all, the tunability of the $1 \mathrm{x} 1$ device is found to be reversible and repeatable, just like the $2 \times 1$ heterostructure studied previously.

The temperature of the $1 \mathrm{x} 1$ device was then brought down to $78 \mathrm{~K}$ and incrementally raised in approximately $25 \mathrm{~K}$ steps up to a maximum of $400 \mathrm{~K}$. At each temperature, three TDTR scans were first collected at zero field (no applied bias). Immediately afterwards, a $-450 \mathrm{kV} \mathrm{cm}^{-1}$ electric field was then applied and held across the 1x1 bilayer device while three more TDTR scans were then collected. All scans were collected at the same spot on the sample due to the spot-to-spot variation observed previously on the $2 \mathrm{x} 1$ bilayers, attributed to the different domain configurations in different areas of the sample. The data was then analyzed using a similar multilayer thermal model to that employed for the room temperature study detailed in [12], substituting the appropriate temperature dependent values for the known parameters in the model $\left(C_{v}, \kappa_{\mathrm{Pt}}\right.$, etc. $)$.

\subsubsection{Results \& Discussion}

Figure 5.7 plots the thermal conductivity of the top tetragonal 30/70 PZT layer within the 1x1 bilayer PZT heterostructure both with and without an applied electric field. It is apparent from the data that the change in thermal conductivity due to the applied field increases as temperature increases, suggesting that the modulation of the fine ferroelastic domain structure has a larger impact on the high-frequency, short-wavelength phonons moving through the bilayer compared to the lower-frequency, longer wavelength ones. As the temperature of the sample decreases, the phonon flux is primarily comprised of phonons with longer wavelengths that are unaffected by the nano domains, and thus show little response to the applied fields.

One of the concerns regarding the data was whether the $-450 \mathrm{kV} \mathrm{cm}^{-1}$ electric fields were actually switching the domain structure at the lowest sample temperatures. Since the 


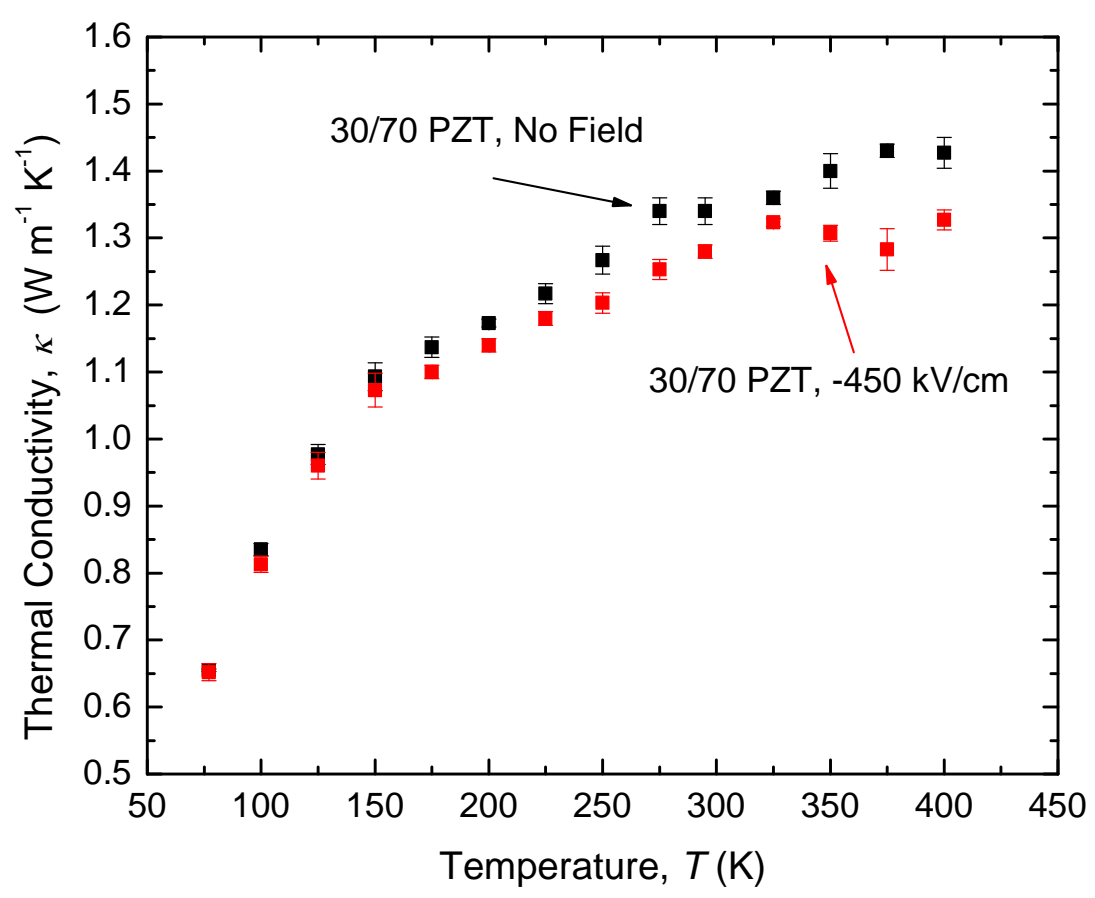

Figure 5.7: Thermal conductivity of the top tetragonal 30/70 PZT layer in the 1x1 bilayer PZT heterostructure over a temperature range of $78-400 \mathrm{~K}$. Measurements were made at the same spot on the sample at every temperature, both with $\left(-450 \mathrm{kV} \mathrm{cm}^{-1}\right.$, red squares $)$ and without $(0 \mathrm{kV}$ $\mathrm{cm}^{-1}$, black squares) an applied electric field. As the temperature increases, the percent change in the thermal conductivity when under bias also increases.

coercive fields $\left( \pm \mathrm{E}_{\mathrm{c}}\right)$ of several PZT materials have been shown to increase with decreasing temperature [133], it was possible that larger electric fields were needed to switch the ferroelastic domain structure within the PZT bilayers at low temperatures. To address this, the ferroelectric hysteresis loops of the same $1 \mathrm{x} 1$ device were measured over the $78-400$ $\mathrm{K}$ temperature range to determine whether or not the applied electrics fields were large enough to switch the domain structure of the bilayer devices.

Figure 5.8 plots these hysteresis loops at several temperatures across the range of interest. In the negative poling regime, the value of $-\mathrm{E}_{\mathrm{c}}$ does indeed increase with decreasing temperature as shown for bulk materials in [133], reaching a maximum of $-178 \mathrm{kV} \mathrm{cm}{ }^{-1}$ at $78 \mathrm{~K}$. However, this $-\mathrm{E}_{\mathrm{c}}$ is considerably lower than the $-450 \mathrm{kV} \mathrm{cm}^{-1}$ field applied to the 


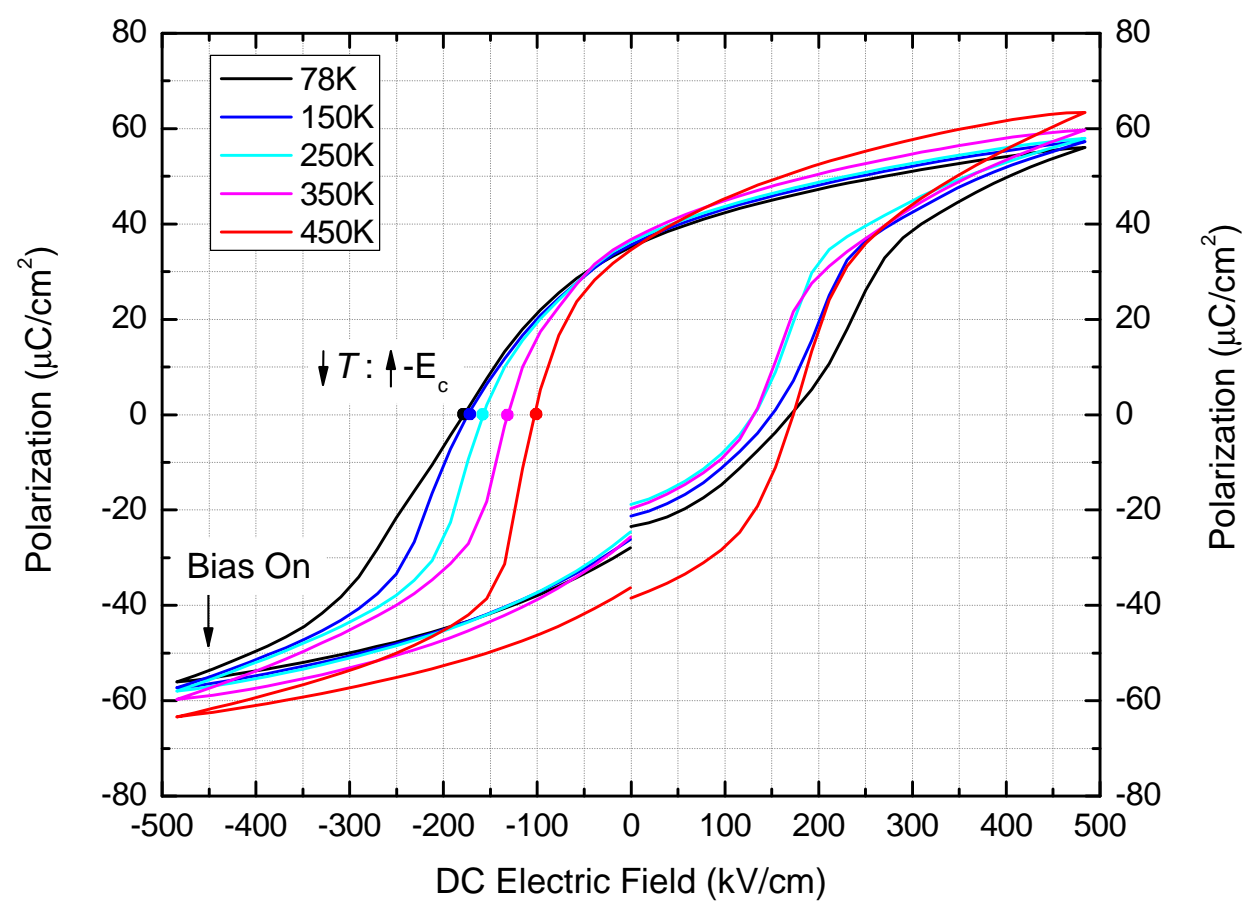

Figure 5.8: Polarization versus electric field (P-E) hysteresis loops measured for the 1x1 bilayer PZT heterostructure at several different temperatures from 78 - $450 \mathrm{~K}$. The data confirms that the $-450 \mathrm{kV} \mathrm{cm}{ }^{-1}$ applied electric fields were indeed large enough to switch the domain structure at all the temperatures tested with TDTR. Furthermore, the value for the coercive field in the negative poling direction $\left(-\mathrm{E}_{\mathrm{c}}\right)$ decreases with increasing temperature, indicating that lower fields are required to switch the domain structure at elevated temperatures.

device, indicating that our applied electric field is large enough to switch the domains at all temperatures. In fact, the hysteresis loops indicate that the $-450 \mathrm{kV} \mathrm{cm}^{-1}$ applied electric field leads to similar saturation polarization $\left( \pm P_{\text {sat }}\right)$ levels, suggesting that the domain structure is fairly similar at all temperatures when a saturating electric field is applied.

Having confirmed that the domain structures within the $1 \mathrm{x} 1$ bilayers were in fact switching at all temperatures, we now shift focus back to the thermal transport properties of the films. One attribute of the data in Figure 5.7 is the fact that the trend resembles that of a heavily disordered solid/system where the thermal conductivity continues to increase with increasing temperature, similar to what was observed for bulk $\mathrm{PbZrO}_{3}$ [23, 24] and thin 
film 30/70 PZT in Chapter 4. Figure 5.9 illustrates this point by plotting the 30/70 PZT data for the $1 \mathrm{x} 1$ bilayer device at no bias field with the single-layer 30/70 PZT data from Chapter 4, as well as the calculated minimum limit for 30/70 PZT $(x=0.7)$ as described in Chapter 4 via Eq. 4.2 and Eq. 4.11.

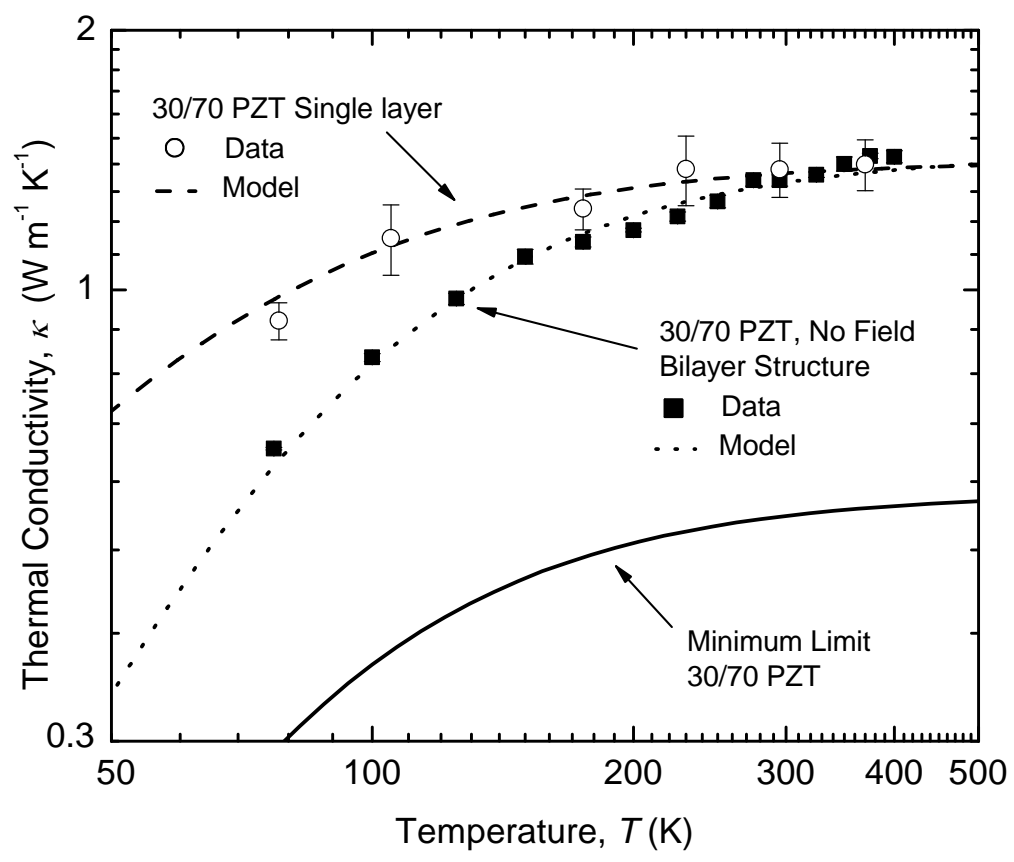

Figure 5.9: Measured thermal conductivity versus temperature for the single layer 30/70 PZT thin film from Chapter 4 along with the data for the top tetragonal 30/70 PZT layer in the bilayer heterostructure. Also plotted is the calculated minimum limit for 30/70 PZT using Eq. 4.11 in Eq. 4.12 with $x=0.7$. The models represent fits to the data defined by Eqs. 4.12 through 4.17 , treating $A$ and $d$ as adjustable parameters to gain insight regarding the phonon scattering processes in these samples.

At temperatures $\geq 300 \mathrm{~K}$, the thermal conductivities of the single layer and bilayer 30/70 PZT are comparable in magnitude, appearing to saturate around a value of 1.4-1.5 W $\mathrm{m}^{-1} \mathrm{~K}^{-1}$ with increasing temperature. This fact suggests that the dominant phonon scattering mechanisms at these temperatures are similar in the two cases. As the temperature decreases from 300 to $80 \mathrm{~K}$, the magnitudes for the thermal conductivity deviate from one another with values for the bilayer 30/70 PZT film decreasing much more rapidly than the 
single layer film.

Interestingly enough, this is similar to the situation examined in Chapter 4 between bulk $\mathrm{PbZrO}_{3}$ and this same single layer 30/70 PZT data. In that case, it was discussed that there must be a strong phonon scattering mechanism present in the single layer 30/70 PZT film to lead to the significant reduction in the thermal conductivity at low temperatures. While the thin film versus bulk argument was a clear possibility, this was addressed through the fits to the data that suggested a much shorter mean free path associated with the boundary scattering term $(d=4.61 \mathrm{~nm})$ compared to either the film thickness or grain size $(325 \mathrm{~nm}$ and $95 \mathrm{~nm}$, respectively).

In the present case with the data in Figure 5.9, we are now comparing two thin film samples of the same PZT composition $(30 / 70, x=0.7)$ and yet we once again see a large difference in the thermal conductivities at lower temperatures. This suggests that the scattering rates of low-frequency, long-wavelength phonons are quite different between the two films. While the physical dimensions and microstructure of the two films are indeed different, this is probably not the root cause because of the fact that the shortest nanostructured length-scales in the two films are quite comparable $(97 \mathrm{~nm}$ average grain size in the single layer 30/70 PZT film; $80 \mathrm{~nm}$ film thickness in the top 30/70 PZT layer of the bilayer). Therefore, we believe that the reduction in thermal conductivity from the scattering of low frequency phonons must be due to a separate effect.

One potential explanation could come from the differences in the ferroelastic domain structures of the two films. Similar to the aforementioned works characterizing the ferroelastic domain structures in similar single-layer 30/70 PZT thin films [104-108], similar techniques have been applied to bilayer PZT samples like those studied here [12, 27, 28, 134-137]. Plan-view piezoresponse force microscopy (PFM) measurements on bilayer PZT heterostructures have also resulted in estimated domain-periodicity of approximately 20-30 nm [27, 104], however it has been further theorized and explored via phase field modeling [135] that there could be an even finer domain structure at the bilayer interface. A finer ferroelastic domain structure near the bilayer PZT heterointerface could explain the 
further reduction in the thermal conductivity of the bilayer sample compared to the single layer film at low temperatures.

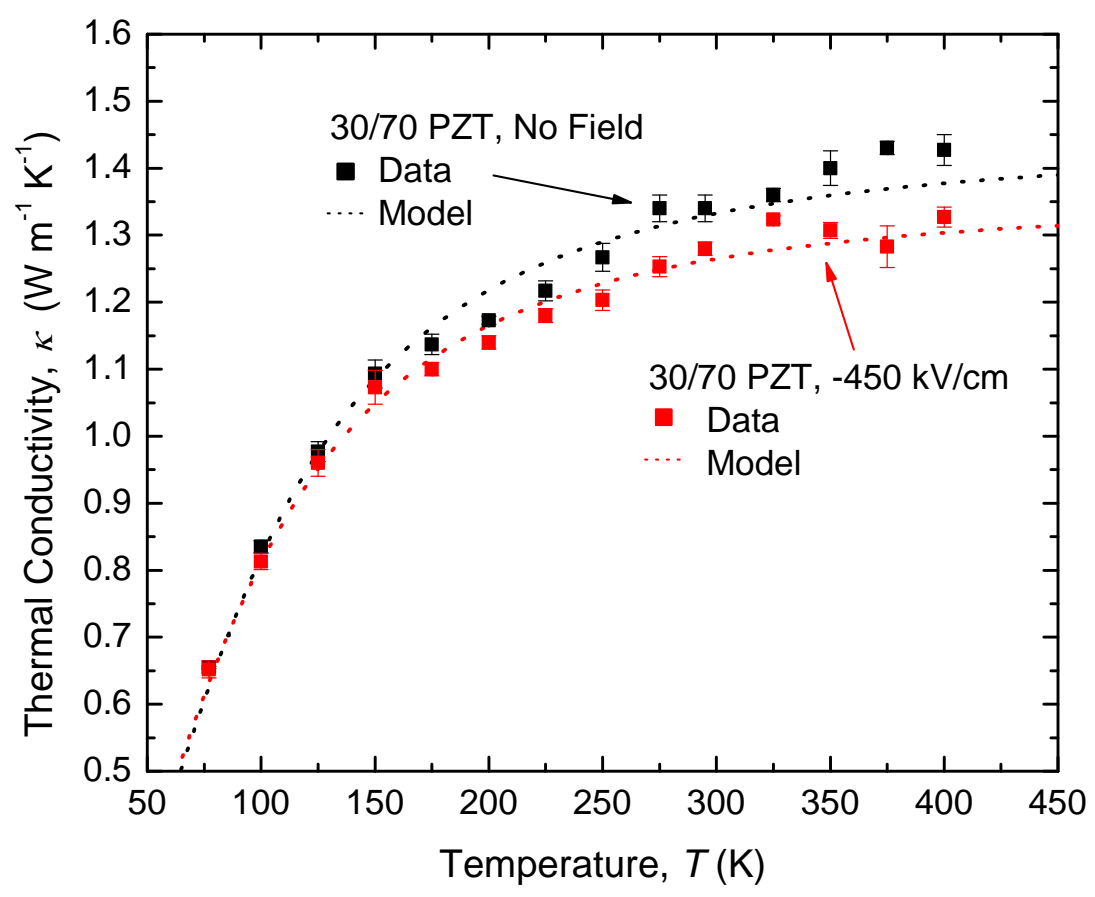

Figure 5.10: Thermal conductivity versus temperature of a 1x1 bilayer PZT device both with and without an applied electric field, along with fits to the data defined by Eqs. 4.12 through 4.17 . treating $A$ and $d$ as adjustable parameters to gain insight regarding the phonon scattering processes in these samples. The change in thermal conductivity due to the applied field increases as temperature increases, suggesting that the modulation of the fine ferroelastic domain structure has a significant impact on the short-wavelength phonons moving through the bilayer.

Having examined the differences in temperature trends between single layer 30/70 PZT and the top layer in the bilayer PZT heterostructure, we return to the data for the bilayer system subjected to an applied electric field. Figure 5.10 replots the data from Figure 5.7 along with the fitted models for each data set using detailed in Chapter 4 using Eq. 4.12 through 4.17 with $x=0.7$ (30/70 PZT). The first thing to note from the data is that there is little to no change in the thermal conductivity due to the applied electric field at low temperatures $(T \leq 150 \mathrm{~K})$. Given the earlier observation of large changes in thermal con- 
ductivity amongst various samples at these same temperatures, the absence of a change in this case suggests that the applied electric field does not impact the dominant phonon scattering mechanism at low temperatures. Alternatively, the change in thermal conductivity due to applied bias mainly occurs at $T>150 \mathrm{~K}$ with the magnitude of the change becoming larger with increasing temperature. Once again, this is different from all the data discussed previously where the thermal conductivity appeared to asymptote to a value of 1.4-1.5 W $\mathrm{m}^{-1} \mathrm{~K}^{-1}$ at high temperatures.

This modulation at $T>150 \mathrm{~K}$ suggests that the decrease in thermal conductivity due to the applied field is driven by a scattering mechanism primarily associated with the higherfrequency phonons. The values for $A$ and $d$ from fitting the model to the data seem to support this as while the value of $d$ increases by about $30 \%$ between the no field and 450 $\mathrm{kV} \mathrm{cm}{ }^{-1}$ cases $\left(d_{0 \mathrm{~V}}=2.68 \mathrm{~nm}, d_{7 \mathrm{~V}}=3.49 \mathrm{~nm}\right)$, the value of $A$ increases by $70 \%\left(A_{0 \mathrm{~V}}=\right.$ $0.297 \times 10^{-42} \mathrm{~s}^{3}, A_{7 \mathrm{~V}}=0.506 \times 10^{-42} \mathrm{~s}^{3}$ ). Given that an increase in $d$ is only expected to lead to an increase in thermal conductivity (shift the model upward) if anything, the change in $A$ is the more significant parameter in capturing the effect of applied fields on the thermal conductivity in the bilayer film. As the parameter $A$ is predominantly associated with the scattering of higher frequency phonons due to the $\omega^{4}$ dependence of Eq. 4.16, this supports the idea that whatever is changing due to the applied fields primarily impacts the higher frequency phonons. Ultimately, additional characterization techniques that are sensitive to the domain wall density within the volume of the the bilayer PZT heterostructure are required to complement these thermal measurements and determine the driving force behind the modulation of the thermal conductivity due to applied electric fields.

\subsection{Summary}

This chapter has detailed our work to create a phonon thermal switch via the modulation of the ferroelastic domain wall density in bilayer PZT thin films using applied electric fields. Based on the earlier work by Anbusathaiah et al. [27, 28], several bilayer PZT het- 
erostructures were fabricated and confirmed to possess mobile ferroelastic domain walls that were able to nucleate/move in response to an applied electric field. Using two different types of electrical probing stations integrated into our TDTR system, the thermal conductivity of the devices was measured both at room temperature and over temperature as a function of applied electric field.

It is interesting to note that from the room temperature measurements, the $2 \times 1$ bilayer device displayed a larger change in thermal conductivity (11\%) than the $1 \times 1$ bilayer device (6\%). As the $2 \times 1$ device possesses a larger top tetragonal layer $(142 \mathrm{~nm})$ than the $1 \times 1$ device $(80 \mathrm{~nm})$, this difference in modulation depth could be due to a size effect associated with the influence of the tetragonal film thickness on the mobility of the ferroelastic domain walls. Furthermore, the large differences in the thermal conductivity at low temperatures between the single layer 30/70 PZT film compared to the bilayer PZT heterostructure are somewhat surprising given the fact that plan-view imaging via PFM estimates that both samples should have similar domain wall periodicity [12, 27, 104]. Given the unique nature of the bilayer system and the significant enhancement in the dielectric and piezoelectric properties that arise, it is possible that the source of the strong phonon scattering at low temperatures is due to a mechanism associated with the bilayer PZT heterointerface. 


\section{Chapter 6}

\section{Summary, Impact \& Future Work}

Dynamic control of the thermal properties is an exciting new frontier in nano/macro scale thermal transport and the concept of an externally-controllable, solid-state thermal switch could prove to be a disruptive advancement with similar scope and impact to that of the solid-state transistor. With this goal in mind, this work has been focused on understanding the intricacies of phonon transport within both the lead zirconate titanate (PZT) material system, as well as the bilayer PZT heterostructure which has been utilized to demonstrate the first electrically-controlled phononic thermal switch at room temperature.

\subsection{Summary}

In Chapter 2, several of the core elements of this dissertation are introduced and defined. The concept of a quantized lattice vibration, or phonon is introduced and discussed with a particular focus on fundamental phonon transport in oxide materials. Domains and domain boundaries of various types (ferroelectric, ferroelastic, etc) are defined and their importance regarding the remarkable properties of several materials is examined. Finally, the influence of domains and domain boundaries on phonon transport that has been reported in the literature to date is presented and discussed.

In Chapter 3, the details regarding the thermal and electrical measurements conducted 
as part of this dissertation are discussed. One of the core outcomes of this work was the demonstrated ability to make thermal transport measurements via time-domain thermoreflectance (TDTR) while the materials and/or devices were subjected to an applied electric field. To accomplish this, two types of electrical probing stations (room-temp and cryotemp) were integrated within the TDTR system to provide accurate thermal measurements in operando.

Chapter 4 introduced the lead zirconate titanate $\left(\mathrm{PbZr}_{1-x} \mathrm{Ti}_{x} \mathrm{O}_{3}, \mathrm{PZT}\right)$ solid solution material system, highlighting its technological importance and the surprising lack of research regarding the thermal transport properties. A comprehensive study of the thermal conductivity of PZT thin films across the compositional phase diagram from $0<x<1$ was completed, with additional measurements made on selected bulk samples. The thermal conductivity of PZT as a function of composition, $x$, does not follow the U-shaped, symmetric trend observed in other alloy/mixture material systems. This asymmetry over $x$ is attributed to the dramatically different thermal properties of the constitutive components in the PZT system; lead zirconate $\left(\mathrm{PbZrO}_{3}\right)$ and lead titanate $\left(\mathrm{PbTiO}_{3}\right)$. Finally, a model for the thermal conductivity of PZT as a function of composition $(\kappa(x))$ is developed and presented. This model agrees quite well with the experimental data for compositions 0 $<x<0.8$, but begins to deviate from the experimental data for compositions $0.8<x<$ 1. This discrepancy for compositions approaching $x=1$ is attributed to the importance of anharmonic phonon scattering effects as the material composition approaches phase-pure $\mathrm{PbTiO}_{3}$.

Chapter 5 detailed the first demonstration of an electrically-controlled phonon thermal switch at room temperature via the modulation of the ferroelastic domain wall density in two different bilayer PZT heterostructures. The modulation of the ferroelastic domain structure ultimately affects the phonon scattering rates within the material, thereby changing the thermal conductivity as a function of applied electric field. Temperature dependent measurements were made on one of the bilayer PZT heterostructures in an attempt to identify the dominant phonon scattering mechanisms. Based on these results, the modulation of 
the domain structure via the applied electric field primarily impacts the higher-frequency phonons, causing the change in thermal conductivity between the zero bias and poled states to increase with increasing temperature.

\subsection{Impact}

This work represents a thorough investigation of the thermal conductivity $\kappa$ for lead zirconate titanate $\left(\mathrm{PbZr}_{1-x} \mathrm{Ti}_{x} \mathrm{O}_{3}, \mathrm{PZT}\right)$ across the entire compositional phase diagram $(0$ $<x<1$ ) and in a variety of forms (bulk, thin film, bilayer heterostructure). Given the technological importance of PZT as a functional material in devices and systems that impact a wide array of industries, this research serves to fill the gap in knowledge regarding the thermal properties. The thermal conductivity of both thin film and bulk PZT vary by a considerable margin as a function of composition, driven by the large difference in the thermal conductivities of the constitutive materials, $\mathrm{PbZrO}_{3}$ and $\mathrm{PbTiO}_{3}$. In fact, the specific cause of the glass-like temperature trend for the thermal conductivity of $\mathrm{PbZrO}_{3}$ remains an open question in the literature. Based on our preliminary analysis of the literature data for $\mathrm{PbZrO}_{3}$ using a semi-classical model for thermal conductivity, we do not believe that the glass-like trend is due to an increase in anharmonic phonon-phonon scattering associated with the large orthorhombic unit cell of the material, indicating that there must be an additionally source of disorder leading to the strong scattering of phonons at all temperatures.

Another important outcome of this work is the observation of a discontinuity in $\kappa$ in the vicinity of the morphotropic phase boundary (MPB, $x=0.48$ ) where there is a 20$25 \%$ decrease in $\kappa$ in our thin film data, as well as the literature data for bulk materials. Given the fact that the MPB composition is one of the most important due to the observed enhancement in the dielectric and piezoelectric properties of PZT materials, it is critical that engineers are aware of this reduction in $\kappa$ and how it may impact their intended application. Furthermore, MPBs exist in several other lead-based [138, 139] and lead-free [140, 141] solid-solution material systems, suggesting that the observations made 
here may apply more broadly to a much wider material space.

Building upon the insight gathered from the work on the PZT material system, Chapter 5 details the first demonstration of an electrically-controllable phononic thermal switch at room temperature. This particular type of thermal switch based on a bilayer PZT heterostructure is particularly remarkable in that the switching media is electrically insulating, compared to other devices that are electrically conductive such as $\mathrm{VO}_{2}$. This may be an important consideration in on-chip thermal management applications requiring high levels of electrical isolation between the active region of the device/chip and the bulk substrate/package.

While the maximum change in thermal conductivity observed to date is quite small compared to other dynamic thermal systems, the work in Chapter 5 aims to explore the engineering space via different heterostructures and temperature dependent measurements with the eventual goal of maximizing the modulation depth of these bilayer PZT thermal devices. It is interesting to note that from the room temperature measurements, the $2 \times 1$ bilayer device displayed a larger change in thermal conductivity (11\%) than the 1x1 bilayer device (6\%). As the $2 \times 1$ device possess a larger top tetragonal layer (142 nm) than the $1 \times 1$ device $(80 \mathrm{~nm})$, this difference in modulation depth could be due to a size effect associated with the influence of the tetragonal film thickness on the mobility of the ferroelastic domain walls.

Furthermore, the large differences in the thermal conductivity at low temperatures between the single layer 30/70 PZT film compared to the bilayer PZT heterostructure are somewhat surprising given the fact that plan-view imaging via PFM estimates that both samples should have similar domain wall periodicity. Given the unique nature of the bilayer system and the significant enhancement in the dielectric and piezoelectric properties that arise, it is possible that the source of the strong phonon scattering at low temperatures is due to a mechanism associated with the bilayer PZT heterointerface. Ultimately, the thermal transport properties of these bilayer PZT devices may provide important clues in the broader body of research concerning the material physics at these heterointerfaces. 


\subsection{Future Work}

There are two major research paths stemming from the work completed in this dissertation. The first is related to the development of an appropriate expression for phonon relaxation/scattering at ferroelastic domain boundaries. In addition to the temperature dependent data measured here for PZT, it would be prudent to measure an additional material with ferroelastic domain walls to aid the development of a general expression that can be applied to various materials. One possible material would be barium titanate $\left(\mathrm{BaTiO}_{3}\right.$, preferably single crystals) due to the large amount of literature available on both the crystalline and ferroelectric properties. In addition to any thermal measurements, extensive characterization of the ferroelastic domain structure via both plan-view techniques like piezoresponse force microscopy (PFM), as well as cross-sectional techniques such at transmission electron microscopy (TEM) will be necessary to accurately determine these structure-property relationships.

The second path for future work concerns the improvement of the bilayer PZT thermal devices to maximize the change in thermal conductivity due to an applied electric field. Given the differences in performance observed for bilayer heterostructures with different thicknesses of tetragonal 30/70 PZT for the top layer, it would be interesting to see if either increasing or decreasing the thickness of the rhombohedral 70/30 PZT bottom layer would have any impact on the modulation depth of the device. In addition to changing the bilayer geometry, it would also be interesting to vary the compositions of the top and bottom layers. It would be particularly interesting to make the top layer out of phase-pure $\mathrm{PbTiO}_{3}(x=1)$ to see if the absence of phonon impurity scattering in this layer would have an impact on either the domain wall mobility and/or the thermal transport properties of the heterostructure. 


\section{Bibliography}

[1] D.-W. Oh, C. Ko, S. Ramanathan, and D. G. Cahill, "Thermal conductivity and dynamic heat capacity across the metal-insulator transition in thin film $\mathrm{VO}_{2}$," Appl. Phys. Lett., vol. 96, no. 15, p. 151906, 2010 .

[2] B. M. Foley, S. Kittiwatanakul, J. C. Duda, J. Lu, and P. E. Hopkins, "Crossover from phonon to electron dominated thermal boundary conductance in vanadium dioxide thin films across the metalinsulator transition." Presented at the Spring Meeting of the Materials Research Society in San Francisco, CA, Spring 2013.

[3] Y. Yan and J. A. Malen, "Periodic heating amplifies the efficiency of thermoelectric energy conversion," Energy Environ. Sci., vol. 6, pp. 1267-1273, 2013.

[4] B. M. Foley, H. J. Brown-Shaklee, J. C. Duda, R. Cheaito, B. J. Gibbons, D. Medlin, J. F. Ihlefeld, and P. E. Hopkins, "Thermal conductivity of nano-grained $\mathrm{SrTiO}_{3}$ thin films," Appl. Phys. Lett., vol. 101, no. 23, p. 231908, 2012.

[5] B. F. Donovan, B. M. Foley, J. F. Ihlefeld, J.-P. Maria, and P. E. Hopkins, "Spectral phonon scattering effects on the thermal conductivity of nano-grained barium titanate," Appl. Phys. Lett., vol. 105, no. 8 , p. $082907,2014$.

[6] D. G. Cahill, "Thermal conductivity measurement from 30 to 750 K: the 3 omega method," Rev. Sci. Instrum., vol. 61, no. 2, pp. 802-808, 1990.

[7] W. Kobayashi, Y. Hayashi, M. Matsushita, Y. Yamamoto, I. Terasaki, A. Nakao, H. Nakao, Y. Murakami, Y. Moritomo, H. Yamauchi, and M. Karppinen, "Anisotropic thermoelectric properties associated with dimensional crossover in quasi-one-dimensional $\mathrm{SrNbO}_{3.4+d}(d \sim 0.03)$," Phys. Rev. B, vol. 84, p. 085118, 2011.

[8] C. Chiritescu, D. G. Cahill, N. Nguyen, D. Johnson, A. Bodapati, P. Keblinski, and P. Zschack, "Ultralow thermal conductivity in disordered, layered WSe 2 crystals," Science, vol. 315, no. 5810, pp. 351-353, 2007.

[9] B. M. Foley, H. J. Brown-Shaklee, M. J. Campion, D. L. Medlin, P. G. Clem, J. F. Ihlefeld, and P. E. Hopkins, "Glass-like thermal conductivity of (010)-textured lanthanum-doped strontium niobate synthesized with wet chemical deposition," J. Am. Ceram. Soc., vol. 98, no. 2, pp. 624-628, 2014.

[10] D. G. Cahill, S. K. Watson, and R. O. Pohl, "Lower limit to the thermal conductivity of disordered crystals," Phys. Rev. B, vol. 46, pp. 6131-6140, 1992.

[11] C.-L. Jia, K. W. Urban, M. Alexe, D. Hesse, and I. Vrejoiu, "Direct observation of continuous electric dipole rotation in flux-closure domains in ferroelectric $\mathrm{Pb}(\mathrm{Zr}, \mathrm{Ti}) \mathrm{O}_{3}$," Science, vol. 331, no. 6023, pp. 1420-1423, 2011. 
[12] J. F. Ihlefeld, B. M. Foley, D. A. Scrymgeour, J. R. Michael, B. B. McKenzie, D. L. Medlin, M. Wallace, S. Trolier-McKinstry, and P. E. Hopkins, "Room-temperature voltage tunable phonon thermal conductivity via reconfigurable interfaces in ferroelectric thin films," Nano Lett., vol. 15, no. 3, pp. 1791-1795, 2015.

[13] A. Mante and J. Volger, "Phonon transport in barium titanate," Physica, vol. 52, no. 4, pp. 577 - 604, 1971.

[14] H. C. Wang, C. L. Wang, W. B. Su, J. Liu, Y. Zhao, H. Peng, J. L. Zhang, M. L. Zhao, J. C. Li, N. Yin, and L. M. Mei, "Enhancement of thermoelectric figure of merit by doping Dy in $\mathrm{La}_{0.1} \mathrm{Sr}_{0.9} \mathrm{TiO}_{3}$ ceramic," Mater. Res. Bull., vol. 45, no. 7, pp. 809 - 812, 2010.

[15] C. Yu, M. L. Scullin, M. Huijben, R. Ramesh, and A. Majumdar, "Thermal conductivity reduction in oxygen-deficient strontium titanates," Appl. Phys. Lett., vol. 92, no. 19, p. 191911, 2008.

[16] P. E. Hopkins, C. Adamo, L. Ye, B. D. Huey, S. R. Lee, D. G. Schlom, and J. F. Ihlefeld, "Effects of coherent ferroelastic domain walls on the thermal conductivity and Kapitza conductance in bismuth ferrite," Appl. Phys. Lett., vol. 102, no. 12, p. 121903, 2013.

[17] P. E. Hopkins, "Thermal transport across solid interfaces with nanoscale imperfections: Effects of roughness, disorder, dislocations, and bonding on thermal boundary conductance," ISRN Mechanical Engineering, vol. 2013, no. 682586, 2013.

[18] B. M. Foley, S. C. Hernández, J. C. Duda, J. T. Robinson, S. G. Walton, and P. E. Hopkins, "Modifying surface energy of graphene via plasma-based chemical functionalization to tune thermal and electrical transport at metal interfaces," Nano Lett., vol. 15, no. 8, pp. 4876-4882, 2015. PMID: 26125524.

[19] K. Nagashio, T. Nishimura, K. Kita, and A. Toriumi, "Contact resistivity and current flow path at metal/graphene contact," Appl. Phys. Lett., vol. 97, no. 14, p. 143514, 2010.

[20] F. Xia, V. Perebeinos, Y.-m. Lin, Y. Wu, and P. Avouris, "The origins and limits of metal-graphene junction resistance," Nat. Nanotechnol., vol. 6, pp. 179-184, 032011.

[21] B. Jaffe, W. R. Cook, and H. Jaffe, Piezoelectric Ceramics. Academic, London, 1971.

[22] M. Tachibana, T. Kolodiazhnyi, and E. Takayama-Muromachi, "Thermal conductivity of perovskite ferroelectrics," Appl. Phys. Lett., vol. 93, no. 9, p. 092902, 2008.

[23] W. N. Lawless, "Glasslike thermal properties of antiferroelectric $\mathrm{PbZrO}_{3}$ at low temperatures," Phys. Rev. B, vol. 30, pp. 6555-6559, Dec 1984.

[24] I. Yoshida, "Thermal conduction in ferroelectric ceramics," J. Phys. Soc. Jpn., vol. 15, no. 12, pp. 2211-2219, 1960.

[25] S. Kallaev, G. Gadzhiev, I. Kamilov, Z. Omarov, S. Sadykov, and L. Reznichenko, "Thermal properties of PZT-based ferroelectric ceramics," Phys. Solid State, vol. 48, pp. 1169-1170, 2006.

[26] S. Lang and E. Ringgaard, "Measurements of the thermal, dielectric, piezoelectric, pyroelectric and elastic properties of porous PZT samples," in Electrical Insulation and Dielectric Phenomena, 2009. CEIDP '09. IEEE Conference on, pp. 739 -742, oct. 2009.

[27] V. Anbusathaiah, D. Kan, F. C. Kartawidjaja, R. Mahjoub, M. A. Arredondo, S. Wicks, I. Takeuchi, J. Wang, and V. Nagarajan, "Labile ferroelastic nanodomains in bilayered ferroelectric thin films," Adv. Mater., vol. 21, no. 34, pp. 3497-3502, 2009. 
[28] V. Anbusathaiah, S. Jesse, M. Arredondo, F. Kartawidjaja, O. Ovchinnikov, J. Wang, S. Kalinin, and V. Nagarajan, "Ferroelastic domain wall dynamics in ferroelectric bilayers," Acta Mater., vol. 58, no. 16 , pp. $5316-5325,2010$.

[29] D. G. Cahill, W. K. Ford, K. E. Goodson, G. D. Mahan, A. Majumdar, H. J. Maris, R. Merlin, and S. R. Phillpot, "Nanoscale thermal transport," J. Appl. Phys., vol. 93, no. 2, pp. 793-818, 2003.

[30] D. G. Cahill, P. V. Braun, G. Chen, D. R. Clarke, S. Fan, K. E. Goodson, P. Keblinski, W. P. King, G. D. Mahan, A. Majumdar, H. J. Maris, S. R. Phillpot, E. Pop, and L. Shi, "Nanoscale thermal transport. ii. 2003-2012," Appl. Phys. Rev., vol. 1, no. 1, pp. -, 2014.

[31] C. W. Chang, D. Okawa, A. Majumdar, and A. Zettl, "Solid-state thermal rectifier," Science, vol. 314, no. 5802, pp. 1121-1124, 2006.

[32] Z. Chen, C. Wong, S. Lubner, S. Yee, J. Miller, W. Jang, C. Hardin, A. Fong, J. E. Garay, and C. Dames, "A photon thermal diode," Nat Commun., vol. 5, 112014.

[33] R. Zheng, J. Gao, J. Wang, and G. Chen, "Reversible temperature regulation of electrical and thermal conductivity using liquid-solid phase transitions," Nat Commun., vol. 2, p. 289, 042011.

[34] J. Zhu, K. Hippalgaonkar, S. Shen, K. Wang, Y. Abate, S. Lee, J. Wu, X. Yin, A. Majumdar, and X. Zhang, "Temperature-gated thermal rectifier for active heat flow control," Nano Lett., vol. 14, no. 8, pp. 4867-4872, 2014.

[35] J. Cho, M. D. Losego, H. G. Zhang, H. Kim, J. Zuo, I. Petrov, D. G. Cahill, and P. V. Braun, "Electrochemically tunable thermal conductivity of lithium cobalt oxide," Nat Commun., vol. 5, 062014.

[36] C. Kittel, Introduction to Solid State Physics. John Wiley and Sons, Inc., Seventh ed., 1996.

[37] G. Chen, Nanoscale Energy Transport and Conversion. Oxford University Press, 2005.

[38] A. J. Minnich, M. S. Dresselhaus, Z. F. Ren, and G. Chen, "Bulk nanostructured thermoelectric materials: current research and future prospects," Energ. Environ. Sci., vol. 2, pp. 466-479, 2009.

[39] R. W. Schwartz, "Chemical solution deposition of perovskite thin films," Chem. Mater, vol. 9, no. 11, pp. 2325-2340, 1997.

[40] R. W. Schwartz, P. G. Clem, J. A. Voigt, E. R. Byhoff, M. Van Stry, T. J. Headley, and N. A. Missert, "Control of microstructure and orientation in solution-deposited $\mathrm{BaTiO}_{3}$ and $\mathrm{SrTiO}_{3}$ thin films," $J$. Am. Ceram. Soc., vol. 82, no. 9, pp. 2359-2367, 1999.

[41] G. L. Brennecka, J. F. Ihlefeld, J.-P. Maria, B. A. Tuttle, and P. G. Clem, "Processing technologies for high-permittivity thin films in capacitor applications," J. Am. Ceram. Soc., vol. 93, no. 12, pp. 39353954, 2010.

[42] Y. Wang, K. Fujinami, R. Zhang, C. Wan, N. Wang, Y. Ba, and K. Koumoto, "Interfacial thermal resistance and thermal conductivity in nanograined $\mathrm{SrTiO}_{3}$," Appl. Phys. Express, vol. 3, no. 3, p. 031101, 2010.

[43] G. Chen, "Thermal conductivity and ballistic-phonon transport in the cross-plane direction of superlattices," Phys. Rev. B, vol. 57, pp. 14958-14973, Jun 1998.

[44] R. A. Cowley, "Lattice dynamics and phase transitions of strontium titanate," Phys. Rev., vol. 134, pp. A981-A997, 051964. 
[45] D.-W. Oh, J. Ravichandran, C.-W. Liang, W. Siemons, B. Jalan, C. M. Brooks, M. Huijben, D. G. Schlom, S. Stemmer, L. W. Martin, A. Majumdar, R. Ramesh, and D. G. Cahill, "Thermal conductivity as a metric for the crystalline quality of $\mathrm{SrTiO}_{3}$ epitaxial layers," Appl. Phys. Lett., vol. 98, no. 22, p. 221904, 2011.

[46] J. Ravichandran, W. Siemons, D. W. Oh, J. T. Kardel, A. Chari, H. Heijmerikx, M. L. Scullin, A. Majumdar, R. Ramesh, and D. G. Cahill, "High-temperature thermoelectric response of double-doped $\mathrm{SrTiO}_{3}$ epitaxial films," Phys. Rev. B., vol. 82, p. 165126, 102010.

[47] J. Ravichandran, W. Siemons, H. Heijmerikx, M. Huijben, A. Majumdar, and R. Ramesh, "An epitaxial transparent conducting perovskite oxide: Double-doped $\mathrm{SrTiO}_{3}$," Chem. Mater, vol. 22, no. 13, pp. 3983-3987, 2010.

[48] N. Ishizawa, F. Marumo, T. Kawamura, and M. Kimura, "The crystal structure of $\mathrm{Sr}_{2} \mathrm{Nb}_{2} \mathrm{O}_{7}$, a compound with perovskite-type slabs," Acta Crystallogr. B, vol. 31, no. 7, pp. 1912-1915, 1975.

[49] D. G. Cahill, A. Melville, D. G. Schlom, and M. A. Zurbuchen, "Low thermal conductivity of $\mathrm{CsBiNb}_{2} \mathrm{O}_{7}$ epitaxial layers," Appl. Phys. Lett., vol. 96, no. 12, p. 121903, 2010.

[50] G. Shabbir and S. Kojima, "Acoustic and thermal properties of strontium pyroniobate single crystals," J. Phys. D, vol. 36, no. 8, p. 1036, 2003.

[51] M. Adachi, Y. Akishige, T. Asahi, K. Deguchi, K. Gesi, K. Hasebe, T. Hikita, T. Ikeda, Y. Iwata, M. Komukae, T. Mitsui, E. Nakamura, N. Nakatani, M. Okuyama, T. Osaka, A. Sakai, E. Sawaguchi, Y. Shiozaki, T. Takenaka, K. Toyoda, T. Tsukamoto, and T. Yagi, " $\mathrm{Sr}_{2} \mathrm{Nb}_{2} \mathrm{O}_{7}$ [F], 8A-5," in LandoltBornstein - Group III Condensed Matter (T. M. Y. Shiozaki, E. Nakamura, ed.), vol. 36A2: Oxides, Springer Materials.

[52] T. D. Sparks, P. A. Fuierer, and D. R. Clarke, "Anisotropic thermal diffusivity and conductivity of La-doped strontium niobate $\mathrm{Sr}_{2} \mathrm{Nb}_{2} \mathrm{O}_{7}$," J. Am. Ceram. Soc., vol. 93, no. 4, pp. 1136-1141, 2010.

[53] P. E. Hopkins and E. S. Piekos, "Lower limit to phonon thermal conductivity of disordered, layered solids," Appl. Phys. Lett., vol. 94, no. 18, p. 181901, 2009.

[54] P. E. Hopkins and T. E. Beechem, "Phonon scattering and velocity considerations in the minimum phonon thermal conductivity of layered solids above the plateau," Nanosc. Microsc. Therm., vol. 14, no. 1, pp. 51-61, 2010.

[55] D. Damjanovic, "Ferroelectric, dielectric and piezoelectric properties of ferroelectric thin films and ceramics," Rep. Prog. Phys., vol. 61, no. 9, p. 1267, 1998.

[56] N. Setter, D. Damjanovic, L. Eng, G. Fox, S. Gevorgian, S. Hong, A. Kingon, H. Kohlstedt, N. Y. Park, G. B. Stephenson, I. Stolitchnov, A. K. Taganstev, D. V. Taylor, T. Yamada, and S. Streiffer, "Ferroelectric thin films: Review of materials, properties, and applications," J. Appl. Phys., vol. 100, no. 5, p. 051606, 2006.

[57] J. F. Ihlefeld, A. Kumar, V. Gopalan, D. G. Schlom, Y. B. Chen, X. Q. Pan, T. Heeg, J. Schubert, X. Ke, P. Schiffer, J. Orenstein, L. W. Martin, Y. H. Chu, and R. Ramesh, "Adsorption-controlled molecular-beam epitaxial growth of $\mathrm{BiFeO}_{3}$," Appl. Phys. Lett., vol. 91, no. 7, p. $071922,2007$.

[58] J. F. Ihlefeld, N. J. Podraza, Z. K. Liu, R. C. Rai, X. Xu, T. Heeg, Y. B. Chen, J. Li, R. W. Collins, J. L. Musfeldt, X. Q. Pan, J. Schubert, R. Ramesh, and D. G. Schlom, "Optical band gap of $\mathrm{BiFeO}_{3}$ grown by molecular-beam epitaxy," Appl. Phys. Lett., vol. 92, no. 14, p. 142908, 2008.

[59] N. Bassiri-Gharb, I. Fujii, E. Hong, S. Trolier-McKinstry, D. V. Taylor, and D. Damjanovic, "Domain wall contributions to the properties of piezoelectric thin films," J. Electroceram., vol. 19, no. 1, pp. 49-67, 2007. 
[60] F. Griggio, S. Jesse, A. Kumar, O. Ovchinnikov, H. Kim, T. N. Jackson, D. Damjanovic, S. V. Kalinin, and S. Trolier-McKinstry, "Substrate clamping effects on irreversible domain wall dynamics in lead zirconate titanate thin films," Phys. Rev. Lett., vol. 108, p. 157604, Apr 2012.

[61] R. Cheaito, C. S. Gorham, A. Misra, K. Hattar, and P. E. Hopkins, "Thermal conductivity measurements via time-domain thermoreflectance for the characterization of radiation induced damage," $J$. Mater. Res., vol. 30, pp. 1403-1412, 2015.

[62] X. Li, W. Cai, J. An, S. Kim, J. Nah, D. Yang, R. Piner, A. Velamakanni, I. Jung, E. Tutuc, S. K. Banerjee, L. Colombo, and R. S. Ruoff, "Large-area synthesis of high-quality and uniform graphene films on copper foils," Science, vol. 324, no. 5932, pp. 1312-1314, 2009.

[63] K. S. Kim, Y. Zhao, H. Jang, S. Y. Lee, J. M. Kim, K. S. Kim, J.-H. Ahn, P. Kim, J.-Y. Choi, and B. H. Hong, "Large-scale pattern growth of graphene films for stretchable transparent electrodes," Nature, vol. 457, pp. 706-710, 022009.

[64] M. Baraket, S. G. Walton, E. H. Lock, J. T. Robinson, and F. K. Perkins, "The functionalization of graphene using electron-beam generated plasmas," Appl. Phys. Lett., vol. 96, no. 23, p. 231501, 2010.

[65] S. C. Hernández, C. J. C. Bennett, C. E. Junkermeier, S. D. Tsoi, F. J. Bezares, R. Stine, J. T. Robinson, E. H. Lock, D. R. Boris, B. D. Pate, J. D. Caldwell, T. L. Reinecke, P. E. Sheehan, and S. G. Walton, "Chemical gradients on graphene to drive droplet motion," ACS Nano, vol. 7, no. 6, pp. 47464755, 2013.

[66] S. C. Hernández, F. J. Bezares, J. T. Robinson, J. D. Caldwell, and S. G. Walton, "Controlling the local chemical reactivity of graphene through spatial functionalization," Carbon, vol. 60, no. 0, pp. 84 $-93,2013$.

[67] O. Balci and C. Kocabas, "Rapid thermal annealing of graphene-metal contact," Appl. Phys. Lett., vol. 101, no. 24, p. 243105, 2012.

[68] J. S. Moon, M. Antcliffe, H. C. Seo, D. Curtis, S. Lin, A. Schmitz, I. Milosavljevic, A. A. Kiselev, R. S. Ross, D. K. Gaskill, P. M. Campbell, R. C. Fitch, K.-M. Lee, and P. Asbeck, "Ultra-low resistance ohmic contacts in graphene field effect transistors," Appl. Phys. Lett., vol. 100, no. 20, p. 203512, 2012.

[69] E. Watanabe, A. Conwill, D. Tsuya, and Y. Koide, "Low contact resistance metals for graphene based devices," Diam. Relat. Mater., vol. 24, no. 0, pp. 171 - 174, 2012.

[70] S. Fujishima, "The history of ceramic filters," IEEE Transactions on Ultrasonics, Ferroelectrics, and Frequency Control, vol. 47, pp. 1-7, Jan 2000.

[71] J. T. Cutchen, J. O. Harris, and G. R. Laguna, "PLZT electrooptic shutters: applications," Appl. Opt., vol. 14, pp. 1866-1873, Aug 1975.

[72] Q. Zhou, S. Lau, D. Wu, and K. K. Shung, "Piezoelectric films for high frequency ultrasonic transducers in biomedical applications," Prog. Mater. Sci., vol. 56, no. 2, pp. 139 - 174, 2011.

[73] G. L. Smith, J. S. Pulskamp, L. M. Sanchez, D. M. Potrepka, R. M. Proie, T. G. Ivanov, R. Q. Rudy, W. D. Nothwang, S. S. Bedair, C. D. Meyer, and R. G. Polcawich, "PZT-based piezoelectric MEMS technology," J. Am. Ceram. Soc., vol. 95, no. 6, pp. 1777-1792, 2012.

[74] N. Izyumskaya, Y.-I. Alivov, S.-J. Cho, H. Morkoç, H. Lee, and Y.-S. Kang, "Processing, structure, properties, and applications of PZT thin films," CRC Cr. Rev. Sol. State, vol. 32, no. 3-4, pp. 111-202, 2007. 
[75] J. Rödel, K. G. Webber, R. Dittmer, W. Jo, M. Kimura, and D. Damjanovic, “Transferring lead-free piezoelectric ceramics into application,” J. Eur. Ceram. Soc., vol. 35, no. 6, pp. 1659 - 1681, 2015.

[76] G. A. Rossetti, J. P. Cline, Y.-M. Chiang, and A. Navrotsky, "Lattice energies and structural distortions in $\mathrm{Pb}\left(\mathrm{Zr}_{x} \mathrm{Ti}_{1-x}\right) \mathrm{O}_{3}$ solid solutions," J. Phys.-Condens. Mat., vol. 14, no. 34, p. 8131, 2002.

[77] M. C. Steele and F. D. Rosi, "Thermal conductivity and thermoelectric power of germanium-silicon alloys," J. Appl. Phys., vol. 29, no. 11, pp. 1517-1520, 1958.

[78] W. Liu and A. A. Balandin, "Thermal conduction in $\mathrm{Al}_{x} \mathrm{Ga}_{1-x} \mathrm{~N}$ alloys and thin films," J. Appl. Phys., vol. 97 , no. 7, p. $073710,2005$.

[79] R. Cheaito, J. C. Duda, T. E. Beechem, K. Hattar, J. F. Ihlefeld, D. L. Medlin, M. A. Rodriguez, M. J. Campion, E. S. Piekos, and P. E. Hopkins, "Experimental investigation of size effects on the thermal conductivity of silicon-germanium alloy thin films," Phys. Rev. Lett., vol. 109, p. 195901, Nov 2012.

[80] W. Wang, H. Sukegawa, and K. Inomata, "Temperature dependence of tunneling magnetoresistance in epitaxial magnetic tunnel junctions using a $\mathrm{Co}_{2} \mathrm{FeAl}$ heusler alloy electrode," Phys. Rev. B, vol. 82, p. 092402, Sep 2010.

[81] G. Rivera-Ruedas, L. M. Ramírez-López, F. Sánchez de Jesús, A. Bolarín-Miró, J. Muñoz-Saldaña, and J. M. Yáñez-Limón, "Thermal characterization of PZT ceramics obtained by mechanically activated mixed oxides using different Pb sources," Int. J. Thermophys., vol. 33, no. 12, pp. 2366-2376, 2012.

[82] R. Varghese, H. Harikrishna, S. T. Huxtable, W. T. Reynolds, and S. Priya, "Effect of crystallinity on thermal transport in textured lead zirconate titanate thin films," ACS Appl. Mater. Interfaces, vol. 6, no. 9, pp. 6748-6756, 2014.

[83] J. F. Ihlefeld, P. G. Kotula, B. D. Gauntt, D. V. Gough, G. L. Brennecka, P. Lu, and E. D. Spoerke, "Solution chemistry, substrate, and processing effects on chemical homogeneity in lead zirconate titanate thin films," J. Am. Ceram. Soc., vol. 98, no. 7, pp. 2028-2038, 2015.

[84] E-112-96, Standard Test Methods for Determining Average Grain Size, vol. 03.01. ASTM International, 2003.

[85] C. Thomsen, J. Strait, Z. Vardeny, H. J. Maris, J. Tauc, and J. J. Hauser, "Coherent phonon generation and detection by picosecond light pulses," Phys. Rev. Lett., vol. 53, pp. 989-992, 1984.

[86] C. Thomsen, H. T. Grahn, H. J. Maris, and J. Tauc, "Surface generation and detection of phonons by picosecond light pulses," Phys. Rev. B, vol. 34, pp. 4129-4138, 1986.

[87] Y. Touloukian and E. Buyco, Thermophysical Properties of Matter - Specific Heat: Metallic Elements and Alloys, vol. 4. New York, IFI/Plenum, 1970.

[88] T. Yoshida, Y. Moriya, T. Tojo, H. Kawaji, T. Atake, and Y. Kuroiwa, "Heat capacity at constant pressure and thermodynamic properties of phase transitions in $\mathrm{PbMO}_{3}(\mathrm{M}=\mathrm{Ti}, \mathrm{Zr}$ and $\mathrm{Hf})$," J. Therm. Anal. Calorim., vol. 95, no. 2, pp. 675-683, 2009.

[89] Y. Kuroiwa, S. Aoyagi, A. Sawada, J. Harada, E. Nishibori, M. Takata, and M. Sakata, "Evidence for $\mathrm{Pb}-\mathrm{O}$ covalency in tetragonal $\mathrm{PbTiO}_{3}$," Phys. Rev. Lett., vol. 87, p. 217601, Nov 2001.

[90] A. M. Glazer, "The classification of tilted octahedra in perovskites," Acta Crystallogr. B., vol. 28, pp. 3384-3392, Nov 1972.

[91] A. M. Glazer, K. Roleder, and J. Dec, "Structure and disorder in single-crystal lead zirconate, PbZrO 3 ," Acta Crystallogr. B., vol. 49, pp. 846-852, Oct 1993. 
[92] D. A. Berlincourt, C. Cmolik, and H. Jaffe, "Piezoelectric properties of polycrystalline lead titanate zirconate compositions," Proceedings of the IRE, vol. 48, pp. 220-229, Feb 1960.

[93] B. Noheda, D. E. Cox, G. Shirane, J. A. Gonzalo, L. E. Cross, and S.-E. Park, "A monoclinic ferroelectric phase in the $\mathrm{Pb}\left(\mathrm{Zr}_{1-x} \mathrm{Ti}_{x}\right) \mathrm{O}_{3}$ solid solution," Appl. Phys. Lett., vol. 74, no. 14, pp. 2059-2061, 1999.

[94] B. Noheda, D. E. Cox, G. Shirane, R. Guo, B. Jones, and L. E. Cross, "Stability of the monoclinic phase in the ferroelectric perovskite $\mathrm{Pb}\left(\mathrm{Zr}_{1-x} \mathrm{Ti}_{x}\right) \mathrm{O}_{3}$," Phys. Rev. B, vol. 63, p. 014103, Dec 2000.

[95] B. Noheda, J. A. Gonzalo, L. E. Cross, R. Guo, S.-E. Park, D. E. Cox, and G. Shirane, "Tetragonalto-monoclinic phase transition in a ferroelectric perovskite: The structure of $\mathrm{PbZr}_{0.52} \mathrm{Ti}_{0.48} \mathrm{O}_{3}$," Phys. Rev. B, vol. 61, pp. 8687-8695, Apr 2000.

[96] A. G. Souza Filho, K. C. V. Lima, A. P. Ayala, I. Guedes, P. T. C. Freire, J. Mendes Filho, E. B. Araújo, and J. A. Eiras, "Monoclinic phase of $\mathrm{PbZr}_{0.52} \mathrm{Ti}_{0.48} \mathrm{O}_{3}$ ceramics: Raman and phenomenological thermodynamic studies," Phys. Rev. B, vol. 61, pp. 14283-14286, Jun 2000.

[97] A. G. Souza Filho, K. C. V. Lima, A. P. Ayala, I. Guedes, P. T. C. Freire, F. E. A. Melo, J. Mendes Filho, E. B. Araújo, and J. A. Eiras, "Raman scattering study of the $\mathrm{PbZr}_{1-x} \mathrm{Ti}_{x} \mathrm{O}_{3}$ system: Rhombohedral-monoclinic-tetragonal phase transitions," Phys. Rev. B, vol. 66, p. 132107, Oct 2002.

[98] J. Frantti, S. Eriksson, S. Hull, V. Lantto, H. Rundlöf, and M. Kakihana, "Composition variation and the monoclinic phase within $\mathrm{Pb}\left(\mathrm{Zr}_{x} \mathrm{Ti}_{1-x}\right) \mathrm{O}_{3}$ ceramics," J. Phys.-Condens. Mat., vol. 15, no. 35, p. $6031,2003$.

[99] M. Kaviany, Heat Transfer Physics. Cambridge University Press, 2008.

[100] D. T. Morelli, J. P. Heremans, and G. A. Slack, "Estimation of the isotope effect on the lattice thermal conductivity of group IV and group III-V semiconductors," Phys. Rev. B, vol. 66, p. 195304, Nov 2002.

[101] B. Abeles, D. S. Beers, G. D. Cody, and J. P. Dismukes, "Thermal conductivity of Ge-Si alloys at high temperatures," Phys. Rev., vol. 125, pp. 44-46, Jan 1962.

[102] B. Abeles, "Lattice thermal conductivity of disordered semiconductor alloys at high temperatures," Phys. Rev., vol. 131, pp. 1906-1911, Sep 1963.

[103] Z. Wang and N. Mingo, "Diameter dependence of SiGe nanowire thermal conductivity," Appl. Phys. Lett., vol. 97, no. 10, p. 101903, 2010.

[104] Y. Ivry, D. Chu, and C. Durkan, "Nanometer resolution piezoresponse force microscopy to study deep submicron ferroelectric and ferroelastic domains," Appl. Phys. Lett., vol. 94, no. 16, p. 162903, 2009.

[105] Y. Ivry, N. Wang, D. Chu, and C. Durkan, " $90^{\circ}$ domain dynamics and relaxation in thin ferroelectric/ferroelastic films," Phys. Rev. B, vol. 81, p. 174118, May 2010.

[106] Y. Ivry, D. P. Chu, and C. Durkan, "Bundles of polytwins as meta-elastic domains in the thin polycrystalline simple multi-ferroic system PZT," Nanotechnology, vol. 21, no. 6, p. 065702, 2010.

[107] Y. Ivry, D. Chu, J. F. Scott, and C. Durkan, "Domains beyond the grain boundary," Adv. Func. Mater, vol. 21, no. 10, pp. 1827-1832, 2011.

[108] Y. Ivry, J. F. Scott, E. K. H. Salje, and C. Durkan, "Nucleation, growth, and control of ferroelectricferroelastic domains in thin polycrystalline films," Phys. Rev. B, vol. 86, p. 205428, Nov 2012. 
[109] E. Sawaguchi, H. Maniwa, and S. Hoshino, "Antiferroelectric structure of lead zirconate," Phys. Rev., vol. 83, pp. 1078-1078, Sep 1951.

[110] J. H. Ko, K. Roleder, and A. Bussmann-Holder, "Determination of elastic stiffness coefficients of lead zirconate single crystals in the cubic phase by brillouin light scattering," IOP Conference Series: Materials Science and Engineering, vol. 54, no. 1, p. 012002, 2014.

[111] A. Y. Wu and R. J. Sladek, "Elastic moduli of $\mathrm{PbZrO}_{3}$ and $\mathrm{PbMg}_{\frac{1}{3}} \mathrm{Nb}_{\frac{2}{3}} \mathrm{O}_{3}$ between 1.5 and $298 \mathrm{k}$ : Anomalies in $\mathrm{PbMg}_{\frac{1}{3}} \mathrm{Nb}_{\frac{2}{3}} \mathrm{O}_{3}$; Debye temperatures and heat-capacity interpretation," Phys. Rev. B, vol. 27, pp. 2089-2091, Feb 1983.

[112] G. Shirane, E. Sawaguchi, and A. Takeda, "On the phase transition in lead zirconate," Phys. Rev., vol. 80, pp. 485-485, Nov 1950.

[113] G. Shirane, S. Hoshino, and K. Suzuki, "X-ray study of the phase transition in lead titanate," Phys. Rev., vol. 80, pp. 1105-1106, Dec 1950.

[114] J. Chen, X. Xing, R. Yu, and G. Liu, "Thermal expansion properties of lanthanum-substituted lead titanate ceramics," J. Am. Ceram. Soc., vol. 88, no. 5, pp. 1356-1358, 2005.

[115] V. A. Chaudhari and G. K. Bichile, "Synthesis, structural and electrical properties of pure $\mathrm{PbTiO}_{3}$ ferroelectric ceramics," Smart Materials Research, vol. 2013, no. 147524, 2013.

[116] Z. Li, M. Grimsditch, C. Foster, and S.-K. Chan, "Dielectric and elastic properties of ferroelectric materials at elevated temperature," J. Phys. Chem. Solids, vol. 57, no. 10, pp. 1433 - 1438, 1996.

[117] A. G. Kalinichev, J. D. Bass, B. N. Sun, and D. A. Payne, "Elastic properties of tetragonal $\mathrm{PbTiO}_{3}$ single crystals by Brillouin scattering," J. Mater. Res., vol. 12, pp. 2623-2627, 101997.

[118] G. Shirane and S. Hoshino, "On the phase transition in lead titanate," J. Phys. Soc. Jpn., vol. 6, no. 4, pp. 265-270, 1951.

[119] J. Long, L. Yang, and X. Wei, "Lattice, elastic properties and Debye temperatures of $\left\{\mathrm{ATiO}_{3}\right\}(\mathrm{A}=$ $\mathrm{Ba}, \mathrm{Ca}, \mathrm{Pb}, \mathrm{Sr}$ ) from first-principles,” J. Alloy Compd., vol. 549, pp. 336 - 340, 2013.

[120] D. G. Cahill, "Analysis of heat flow in layered structures for time-domain thermoreflectance," Rev. Sci. Instrum., vol. 75, no. 12, pp. 5119-5122, 2004.

[121] A. J. Schmidt, X. Chen, and G. Chen, "Pulse accumulation, radial heat conduction, and anisotropic thermal conductivity in pump-probe transient thermoreflectance," Rev. Sci. Instrum., vol. 79, no. 11, p. $114902,2008$.

[122] P. E. Hopkins, L. M. Phinney, J. R. Serrano, and T. E. Beechem, "Effects of surface roughness and oxide layer on the thermal boundary conductance at aluminum/silicon interfaces," Phys. Rev. B, vol. 82, p. 085307, 2010.

[123] Y. S. Touloukian and E. H. Buyco, Thermophysical Properties of Matter - Specific Heat: Nonmetallic Solids, vol. 5. New York, IFI/Plenum, 1970.

[124] C. Y. Ho, R. W. Powell, and P. E. Liley, "Thermal conductivity of the elements," J. Phys. Chem. Ref. Data, vol. 1, no. 2, pp. 279-421, 1972.

[125] Q. G. Zhang, B. Y. Cao, X. Zhang, M. Fujii, and K. Takahashi, "Influence of grain boundary scattering on the electrical and thermal conductivities of polycrystalline gold nanofilms," Phys. Rev. B, vol. 74, p. 134109, 2006. 
[126] X. Zhang, H. Xie, M. Fujii, H. Ago, K. Takahashi, T. Ikuta, H. Abe, and T. Shimizu, "Thermal and electrical conductivity of a suspended platinum nanofilm," Appl. Phys. Lett., vol. 86, no. 17, p. $171912,2005$.

[127] G. A. Rossetti and A. Navrotsky, "Calorimetric investigation of tricritical behavior in tetragonal $\mathrm{Pb}\left(\mathrm{Zr}_{x} \mathrm{Ti}_{1-x}\right) \mathrm{O}_{3}$," J. Solid State Chem., vol. 144, no. 1, pp. 188 - 194, 1999.

[128] A. J. Schmidt, R. Cheaito, and M. Chiesa, "A frequency-domain thermoreflectance method for the characterization of thermal properties," Rev. Sci. Instrum., vol. 80, no. 9, p. 094901, 2009.

[129] X. Xu, C. Randorn, P. Efstathiou, and J. T. S. Irvine, “A red metallic oxide photocatalyst,” Nat. Mater., vol. 11, pp. 595-598, 072012.

[130] A. S. Mischenko, Q. Zhang, J. F. Scott, R. W. Whatmore, and N. D. Mathur, "Giant electrocaloric effect in thin-film $\mathrm{PbZr}_{0.95} \mathrm{Ti}_{0.05} \mathrm{O}_{3}$," Science, vol. 311, no. 5765, pp. 1270-1271, 2006.

[131] Y. Ehara, S. Yasui, J. Nagata, D. Kan, V. Anbusathaiah, T. Yamada, O. Sakata, H. Funakubo, and V. Nagarajan, "Ultrafast switching of ferroelastic nanodomains in bilayered ferroelectric thin films," Appl. Phys. Lett., vol. 99, no. 18, p. 182906, 2011.

[132] R. Mahjoub, V. Anbusathaiah, S. P. Alpay, and V. Nagarajan, "Ferroelastic domains in bilayered ferroelectric thin films," J. Appl. Phys., vol. 104, no. 12, p. 124103, 2008.

[133] M. W. Hooker, "Properties of PZT-based piezoelectric ceramics between -150 and $250^{\circ}$ c," Tech. Rep., NASA Langley Research Center, Hampton, VA, 1998.

[134] S. Wicks, K. Seal, S. Jesse, V. Anbusathaiah, S. Leach, R. E. Garcia, S. V. Kalinin, and V. Nagarajan, "Collective dynamics in nanostructured polycrystalline ferroelectric thin films using local time-resolved measurements and switching spectroscopy," Acta Mater, vol. 58, no. 1, pp. 67 - 75 , 2010.

[135] F. Xue, J. Wang, G. Sheng, E. Huang, Y. Cao, H. Huang, P. Munroe, R. Mahjoub, Y. Li, V. Nagarajan, and L. Chen, "Phase field simulations of ferroelectrics domain structures in $\mathrm{PbZr}_{x} \mathrm{Ti}_{1-x} \mathrm{O}_{3}$ bilayers," Acta Mater., vol. 61, no. 8, pp. 2909 - 2918, 2013.

[136] H.-H. Huang, Q. Zhang, E. Huang, R. Maran, O. Sakata, Y. Ehara, T. Shiraishi, H. Funakubo, P. Munroe, and N. Valanoor, "Epitaxial $\mathrm{PbZr}_{x} \mathrm{Ti}_{1-x} \mathrm{O}_{3}$ ferroelectric bilayers with giant electromechanical properties," Adv. Mater. Interf., vol. 2, no. 8, p. 1500075, 2015.

[137] G. Liu, Q. Zhang, H.-H. Huang, P. Munroe, V. Nagarajan, H. Simons, Z. Hong, and L.-Q. Chen, "Reversible polarization rotation in epitaxial ferroelectric bilayers," Adv. Mater. Interf., p. 1600444, 2016.

[138] D. La-Orauttapong, B. Noheda, Z.-G. Ye, P. M. Gehring, J. Toulouse, D. E. Cox, and G. Shirane, "Phase diagram of the relaxor ferroelectric $(1-x) \mathrm{Pb}\left(\mathrm{Zn}_{1 / 3} \mathrm{Nb}_{2 / 3}\right) \mathrm{O}_{3}-x \mathrm{PbTiO}_{3}$," Phys. Rev. B, vol. 65, p. 144101, Mar 2002.

[139] B. Noheda, D. E. Cox, G. Shirane, J. Gao, and Z.-G. Ye, "Phase diagram of the ferroelectric relaxor $(1-x) \mathrm{PbMg}_{1 / 3} \mathrm{Nb}_{2 / 3} \mathrm{O}_{3}-x \mathrm{PbTiO}_{3}$," Phys. Rev. B, vol. 66, p. 054104, Aug 2002.

[140] Y. Saito, H. Takao, T. Tani, T. Nonoyama, K. Takatori, T. Homma, T. Nagaya, and M. Nakamura, “Lead-free piezoceramics," Nature, vol. 432, pp. 84-87, 112004.

[141] F.-Z. Yao, K. Wang, L.-Q. Cheng, X. Zhang, W. Zhang, F. Zhu, and J.-F. Li, "Nanodomain engineered $(\mathrm{K}, \mathrm{Na}) \mathrm{NbO}_{3}$ lead-free piezoceramics: Enhanced thermal and cycling reliabilities," J. Am. Ceram. Soc., vol. 98, no. 2, pp. 448-454, 2015. 
[142] Y. L. Tang, Y. L. Zhu, X. L. Ma, A. Y. Borisevich, A. N. Morozovska, E. A. Eliseev, W. Y. Wang, Y. J. Wang, Y. B. Xu, Z. D. Zhang, and S. J. Pennycook, "Observation of a periodic array of flux-closure quadrants in strained ferroelectric $\mathrm{PbTiO}_{3}$ films," Science, vol. 348, no. 6234, pp. 547-551, 2015.

[143] C. T. Nelson, B. Winchester, Y. Zhang, S.-J. Kim, A. Melville, C. Adamo, C. M. Folkman, S.-H. Baek, C.-B. Eom, D. G. Schlom, L.-Q. Chen, and X. Pan, "Spontaneous vortex nanodomain arrays at ferroelectric heterointerfaces," Nano Lett., vol. 11, no. 2, pp. 828-834, 2011. 


\section{Appendix A}

\section{TDTR at Multiple Modulation Frequencies in Bilayer PZT}

\section{Heterostructures}

In the experimental investigation of Section 5.1, it was found that lower modulation frequencies were necessary to observe any measurable change in the thermal conductivity of the bilayer PZT heterostructure due to applied bias. As the thermal penetration depth $(\delta)$ in the TDTR measurement is related to the modulation frequency of the pump beam through the equation $\delta=\sqrt{\kappa /\left(\pi f C_{V}\right)}$ (where $\kappa$ is the thermal conductivity, $f$ is the pump modulation frequency and $C_{V}$ is the volumetric heat capacity), this observation suggests that there may be certain features within the bilayer PZT heterostructure that perform the majority of the phonon scattering as opposed to it being a homogenous effect.



Figure 1: Diagram of the $3 \times 1$ bilayer PZT device used in these TDTR measurements with multiple thermal modulation frequencies.

In an attempt to explore this effect in the frequency space, measurements were conducted on a 3x1 bilayer PZT heterostructure over fifteen thermal modulation frequencies logarithmically spaced from $700 \mathrm{kHz}$ to $9 \mathrm{MHz}$. Figure 1 illustrates the geometry of the 
$3 \times 1$ bilayer PZT device employed for these measurements. Figure 2(a) depicts the relationship between modulation frequency and $\delta$, with $\delta$ decreasing with increasing modulation frequency. Figure 2 (b) plots the 2D surface of TDTR ratio data collected at the fifteen different modulation depths, estimated using the aforementioned expression based on the modulation frequencies. By inspecting the percent change in this surface between the unpoled state and both actively poled and post-poled states, respectively, we hope to determine which modulation frequencies produce the largest changes in TDTR signal and hypothesize why this is the case.
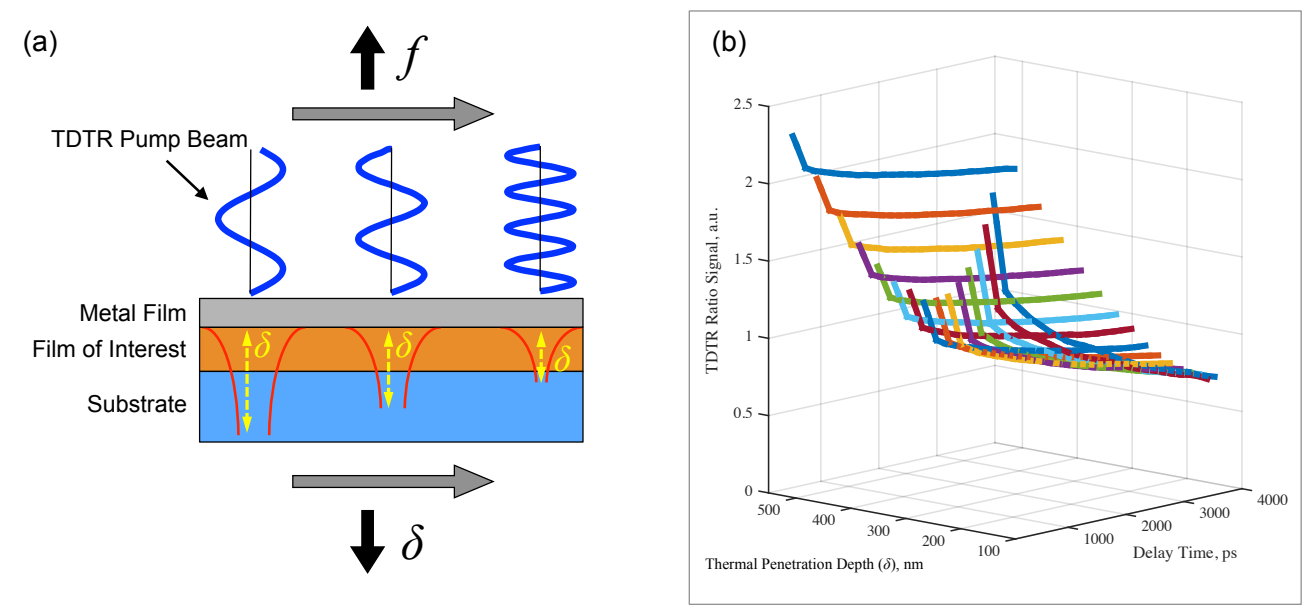

Figure 2: (a) Diagram of the relationship between thermal penetration depth, $\delta$, and thermal modulation frequency; as the thermal modulation frequency increases, $1 / e$ value of $\delta$ decreases. (b) $2 \mathrm{D}$ surface of TDTR ratio data collected by making full TDTR scans at fifteen modulation frequencies ranging from $700 \mathrm{kHz}$ to $9 \mathrm{MHz}$.

Figure 3 plots the percent change in the 2D TDTR ratio surface while an electric field is actively applied to a previously unpoled bilayer PZT device (a), as well as after the electric field has been removed (b). During active poling of the bilayer PZT device, the largest changes in the TDTR ratio surface appear to be at the shallow and deeper penetration depths (highest and lowest modulation frequencies, respectively), with maximum changes of $-3 \%$ observed. This decrease in the signal due to applied bias is consistent with the measurements reported earlier in this dissertation and relates to the decrease in the thermal conductivity of the bilayer PZT device while under applied bias. Little to no change 
appears to occur in the central modulation depths of the range. When the electric field is removed, the TDTR signals at deeper penetration depths (lower frequencies) recover to their unpoled levels (i.e., the percent change goes to zero). This suggests that the features of the bilayer PZT device that cause the changes at these depths are reversible in nature, as was demonstrated in Sections 5.1 and 5.2. Conversely, the TDTR signals at the shallower penetration depths do not recover to their unpoled levels when the electric field is removed. This suggests that the characteristics of the bilayer PZT device associated with these changes are irreversible and persist after poling the device.
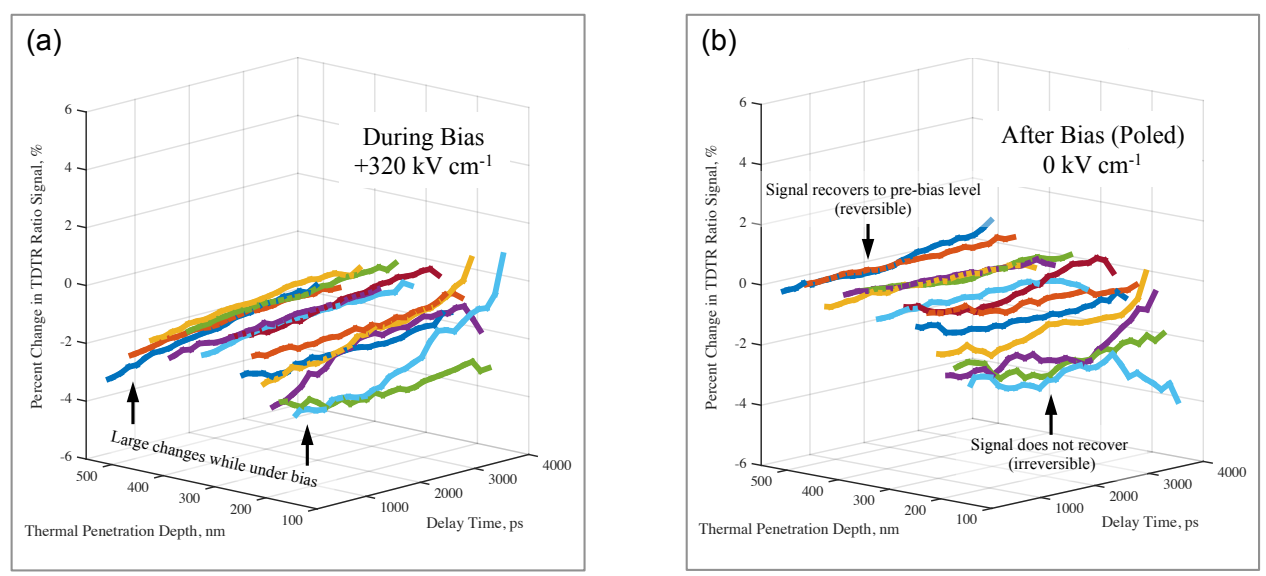

Figure 3: Percent change in the 2D TDTR ratio surface compared to data collected on an unpoled bilayer PZT device both (a) during active bias and (b) after the bias is removed. During active poling of the bilayer PZT device, the largest changes in the TDTR ratio surface appear to be at the shallow and deeper penetration depths (highest and lowest modulation frequencies, respectively), with maximum changes of $-3 \%$ observed. Little to no change appears to occur in the central modulation depths of the range. When the electric field is removed, the TDTR signals at deeper penetration depths (lower frequencies) recover to their unpoled levels (i.e., the percent change goes to zero) while the TDTR signals at the shallower penetration depths do not recover to their unpoled levels. This suggests that the features of the bilayer PZT device that cause the changes at deeper depths are reversible in nature while the changes at shallower depths are irreversible.

To lend insight as to what aspects of the bilayer PZT device could be driving these reversible and irreversible changes, Figure 4 plots the sensitivity of the 2D TDTR ratio surface (calculated using the 4-layer thermal model detailed in Chapter 4) to a $10 \%$ decrease in both (a) the thermal conductivity of the top tetragonal 30/70 PZT layer and (b) 
the thermal conductivity of the bottom rhombohedral 70/30 PZT layer. As discussed in the dissertation, the domain structure of the 70/30 PZT bottom layer is heavily clamped and not expected to contribute to the modulation of thermal conductivity in the bilayer device. However, we include it here to illustrate how the model would respond to a change due to a deeper feature in the device compared to a shallower feature. In Figure 4(a), we see that a $10 \%$ decrease in the thermal conductivity of the top layer is expected to change the TDTR signal at all delay times for most of the modulation depths, with the largest changes occurring at measurement depths of approximately $2 \mathrm{MHz}$. Figure $4(\mathrm{~b})$ shows that a $10 \%$ change in the thermal conductivity of the bottom layer should also cause a sizable change of -2 to $-3 \%$ at deeper modulation depths, however there should be little to no change in the TDTR signal at shallower penetration depths. While these results indicate that the large changes in TDTR signal at deeper penetration depths can be due to changes in either layer, the changes at shallower depths are likely to be due to changes in the top tetragonal PZT layer and not the bottom layer. What this means with regards to the data in Figure 3 (b) is that the irreversible change in the 2D TDTR ratio surface is likely due to the ferroelastic domain structure in the top tetragonal layer only.

While this data is largely for exploratory purposes and more experiments are certainly necessary, it is a very interesting result which suggests that with (a) finer depth resolution and (b) the combined analysis of both the depth (frequency domain) and time domain data, we may be able to isolate/enhance the sensitivity of this technique when measurement complex multilayer structures, like bilayer PZT devices. Furthermore, this approach may be useful as an alternative to TEM when probing domain dynamics in a variety of other thin film systems such as flux-closure [11, 142] and vortex nano domains [143] at oxide heterointerfaces. 

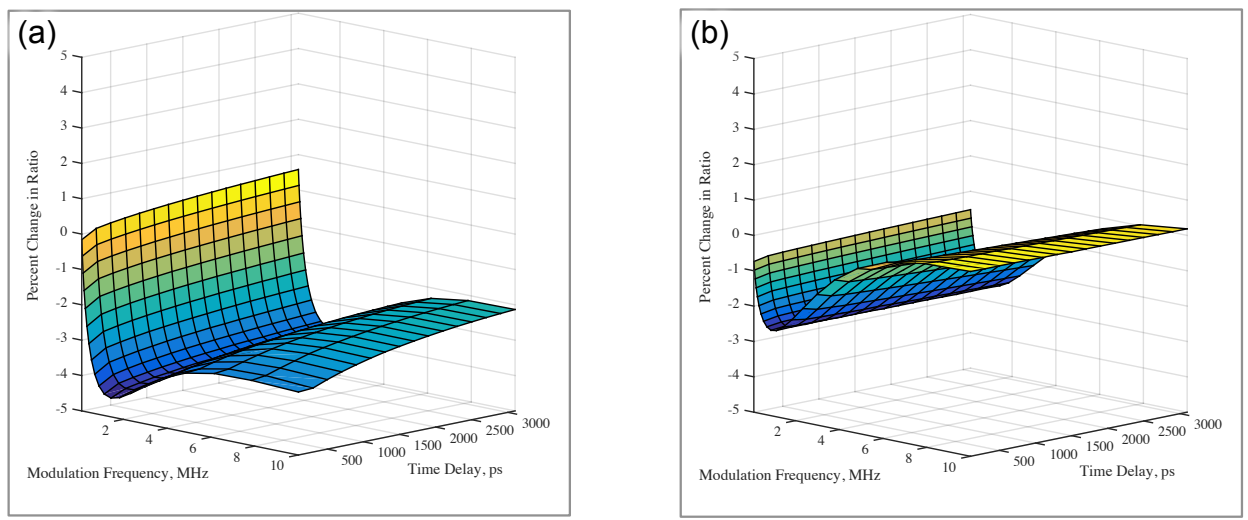

Figure 4: Sensitivity of the 4-layer thermal model detailed in Chapter 4 to $10 \%$ decreases in both (a) the thermal conductivity of the top tetragonal PZT layer $\left(\kappa_{2}\right)$ and $(b)$ the bottom rhombohedral PZT layer $\left(\kappa_{3}\right)$. While these results indicate that the large changes in TDTR signal at deeper penetration depths can be due to changes in either layer, the changes at shallower depths are likely to be due to changes in the top tetragonal PZT layer and not the bottom layer, meaning that the irreversible change in the 2D TDTR ratio surface in Figure 3 (b) is likely due to the ferroelastic domain structure in the top tetragonal layer only. 